\title{
Groundwater Level Forecasting with Artificial Neural Networks: A Comparison of LSTM, CNN and NARX (Supplementary Material)
}

Wunsch, A., Liesch, T., Broda, S.

Content Overview:

- Table S1 lists all hydrographs including information such as identifiers and coordinates

- Tables S2 to S4 summarize the results of the hyperparameter optimization for all models

- Tables S5 and S6 give information on the forecast accuracy of all models

- Fig. S1 to S34: Seq2Val Results

- Fig. S35 to S68: Seq2Seq Results

- Figure S69 explores the robustness of the models in terms of ensemble error variance

- Figures S70 to S72 give further details on how the length of the training data set influences the seq2val model performance. Figure S73 examines the same effect when training ends even 5 years earlier and Fig. S74 examines the development of several statistic measures in a comparable set up

\section{List of Tables}

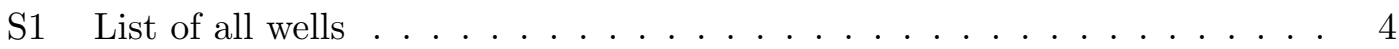

S2 NARX Hyperparameters . . . . . . . . . . . . . . . . . . . 5

S3 LSTM Hyperparameters . . . . . . . . . . . . . . . . . . . . . 6

S4 CNN Hyperparameters . . . . . . . . . . . . . . . . . 7

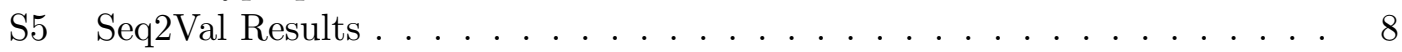

S6 Seq2Seq Results . . . . . . . . . . . . . . . . . . . 9

\section{List of Figures}

S1 Seq2Val test results for well BW_1-115-8 . . . . . . . . . . . . . 10

S2 Seq2Val test results for well BW_103-261-4 . . . . . . . . . . . . . 11

S3 Seq2Val test results for well BW_104-114-5 . . . . . . . . . . . . . 12

S4 Seq2Val test results for well BW_104-307-0 . . . . . . . . . . . . 13 
S5 Seq2Val test results for well BW_138-019-0 . . . . . . . . . . . . . 14

S6 Seq2Val test results for well BW_157-260-8 . . . . . . . . . . . . . 15

S7 Seq2Val test results for well BW_710-256-3 . . . . . . . . . . . . 16

S8 Seq2Val test results for well BW_765-306-3 . . . . . . . . . . . . . 17

S9 Seq2Val test results for well BW_781-304-2 . . . . . . . . . . . . . 18

S10 Seq2Val test results for well BW_1851-305-1 . . . . . . . . . . . . . . 19

S11 Seq2Val test results for well HE_11874 . . . . . . . . . . . . . . . . . 20

S12 Seq2Val test results for well HE_12905 . . . . . . . . . . . . . . . . . 21

S13 Seq2Val test results for well HE_12946 . . . . . . . . . . . . . . . . 22

S14 Seq2Val test results for well HE_13490 . . . . . . . . . . . . . . . 23

S15 Seq2Val test results for well HE_13518 . . . . . . . . . . . . . . . . . 24

S16 Seq2Val test results for well RP_2375118200 . . . . . . . . . . . . 25

S17 Seq2Val test results for well RP_2375283300 . . . . . . . . . . . . . 26

S18 Seq2Val GWL $\mathrm{GW}_{t-1}$ test results for well BW_1-115-8 . . . . . . . . . . 27

S19 Seq2Val GWL $\mathrm{G}_{t-1}$ test results for well BW_103-261-4 . . . . . . . . . . 28

S20 Seq2Val GWL $\mathrm{GW}_{t-1}$ test results for well BW_104-114-5 . . . . . . . . . . 29

S21 Seq2Val GWL $\mathrm{GW}_{t-1}$ test results for well BW_104-307-0 . . . . . . . . . . . 30

S22 Seq2Val GWL $\mathrm{GW}_{t-1}$ test results for well BW_138-019-0 . . . . . . . . . . 31

S23 Seq2Val GWL $\mathrm{GW}_{t-1}$ test results for well BW_157-260-8 . . . . . . . . . . . 32

S24 Seq2Val GWL $\mathrm{GW}_{t-1}$ test results for well BW_710-256-3 . . . . . . . . . . 33

S25 Seq2Val GWL $\mathrm{GW}_{t-1}$ test results for well BW_765-306-3 . . . . . . . . . . 34

S26 Seq2Val GWL $\mathrm{GW}_{t-1}$ test results for well BW_781-304-2 . . . . . . . . . . . 35

S27 Seq2Val GWL $\mathrm{GW}_{t-1}$ test results for well BW_1851-305-1 . . . . . . . . . . 36

S28 Seq2Val GWL $\mathrm{GW}_{t-1}$ test results for well HE_11874 . . . . . . . . . . . . . . 37

S29 Seq2Val GWL $\mathrm{GW}_{t-1}$ test results for well HE_12905 . . . . . . . . . . . . . 38

S30 Seq2Val GWL $\mathrm{GW}_{t-1}$ test results for well HE_12946 . . . . . . . . . . . . . . . 39

S31 Seq2Val GWL $\mathrm{GW}_{t-1}$ test results for well HE_13490 . . . . . . . . . . . . . 40

S32 Seq2Val GWL $\mathrm{GW}_{t-1}$ test results for well HE_13518 . . . . . . . . . . . . . 41

S33 Seq2Val GWL $\mathrm{G}_{t-1}$ test results for well RP_2375118200 . . . . . . . . . . . 42

S34 Seq2Val GWL $\mathrm{GW}_{t-1}$ test results for well RP_2375283300 . . . . . . . . . . 43

S35 Seq2Seq test results for well BW_1-115-8 . . . . . . . . . . . . . . 44

S36 Seq2Seq test results for well BW_103-261-4 . . . . . . . . . . . . . 45

S37 Seq2Seq test results for well BW_104-114-5 . . . . . . . . . . . . . 46

S38 Seq2Seq test results for well BW_104-307-0 . . . . . . . . . . . . . . 47

S39 Seq2Seq test results for well BW_138-019-0 . . . . . . . . . . . . . 48

S40 Seq2Seq test results for well BW_157-260-8 . . . . . . . . . . . . . . . 49

S41 Seq2Seq test results for well BW_710-256-3 . . . . . . . . . . . . 50

S42 Seq2Seq test results for well BW_765-306-3 . . . . . . . . . . . . . 51

S43 Seq2Seq test results for well BW_781-304-2 . . . . . . . . . . . . . 52

S44 Seq2Seq test results for well BW_1851-305-1 . . . . . . . . . . . . . 53

S45 Seq2Seq test results for well HE_11874 . . . . . . . . . . . . . . . . 54

S46 Seq2Seq test results for well HE_12905 . . . . . . . . . . . . . . . 55

S47 Seq2Seq test results for well HE_12946 . . . . . . . . . . . . . . . 56

S48 Seq2Seq test results for well HE_13490 . . . . . . . . . . . . . 57 
S49 Seq2Seq test results for well HE_13518 . . . . . . . . . . . . . . . 58

S50 Seq2Seq test results for well RP_2375118200 . . . . . . . . . . . . . 59

S51 Seq2Seq test results for well RP_2375283300 . . . . . . . . . . . . . 60

S52 Seq2Seq GWL $\mathrm{GW}_{t-1}$ test results for well BW_1-115-8 . . . . . . . . . . 61

S53 Seq2Seq GWL $\mathrm{GW}_{t-1}$ test results for well BW_103-261-4 . . . . . . . . . . 62

S54 Seq2Seq GWL $\mathrm{GW}_{t-1}$ test results for well BW_104-114-5 . . . . . . . . . . 63

S55 Seq2Seq GWL $\mathrm{GW}_{t-1}$ test results for well BW_104-307-0 . . . . . . . . . . . . 64

S56 Seq2Seq GWL $\mathrm{GW}_{t-1}$ test results for well BW_138-019-0 . . . . . . . . . . 65

S57 Seq2Seq GWL $\mathrm{GW}_{t-1}$ test results for well BW_157-260-8 . . . . . . . . . 66

S58 Seq2Seq $\mathrm{GWL}_{t-1}$ test results for well BW_710-256-3 . . . . . . . . . . 67

S59 Seq2Seq GWL GW-1 $_{t}$ test results for well BW_765-306-3 . . . . . . . . . 68

S60 Seq2Seq GWL $\mathrm{GW}_{t-1}$ test results for well BW_781-304-2 . . . . . . . . . . . 69

S61 Seq2Seq GWL $\mathrm{GW}_{t-1}$ test results for well BW_1851-305-1 . . . . . . . . . 70

S62 Seq2Seq GWL $\mathrm{GW}_{t-1}$ test results for well HE_11874 . . . . . . . . . . . . . 71

S63 Seq2Seq GWL $\mathrm{G}_{t-1}$ test results for well HE_12905 . . . . . . . . . . . . . . 72

S64 Seq2Seq GWL $\mathrm{G}_{t-1}$ test results for well HE_12946 . . . . . . . . . . . . . 73

S65 Seq2Seq GWL $\mathrm{G}_{t-1}$ test results for well HE_13490 . . . . . . . . . . . . . 74

S66 Seq2Seq GWL $\mathrm{G}_{t-1}$ test results for well HE_13518 . . . . . . . . . . . . . 75

S67 Seq2Seq GWL $\mathrm{G}_{t-1}$ test results for well RP_2375118200 . . . . . . . . . 76

S68 Seq2Seq GWL $\mathrm{GW}_{t-1}$ test results for well RP_2375283300 . . . . . . . . . . 77

S69 Model Robustness . . . . . . . . . . . . . . . . . . . . . 78

S70 Influence of time series length on Seq2Val Models . . . . . . . . . . . . . 79

S71 Influence of time series length on Seq2Val GWLt-1 Models . . . . . . . . . 79

S72 Influence of time series length on all Seq2Val Models with shorter training 80

S73 Influence of time series length on statistic measures . . . . . . . . . . . . 80 
Table S1: List of all wells included in the dataset. ID refers to the respective data web service.

\begin{tabular}{|l|l|l|l|l|l|l|}
\hline Dataset_ID & ID & Name & X & Y & Coord.Sys. & Country \\
\hline BW_1-115-8 & $1 / 115-8$ & 1115 SCHUTTERWALD & 416098 & 5369591 & ETRS_1989_UTM_Zone_32N & Germany \\
\hline BW_103-261-4 & $103 / 261-4$ & MALSCH & 452062 & 5415349 & ETRS_1989_UTM_Zone_32N & Germany \\
\hline BW_104-114-5 & $104 / 114-5$ & 1807 RENCHEN 1 & 425124 & 5383055 & ETRS_1989_UTM_Zone_32N & Germany \\
\hline BW_104-307-0 & $104 / 307-0$ & GWM SK 22 - 3021A, Reilingen & 468049 & 5458156 & ETRS_1989_UTM_Zone_32N & Germany \\
\hline BW_138-019-0 & $138 / 019-0$ & FLB 5 Breisach-Hochstetten & 396354 & 5319363 & ETRS_1989_UTM_Zone_32N & Germany \\
\hline BW_157-260-8 & $157 / 260-8$ & 1555 BRUCHHAUSEN & 453043 & 5418954 & ETRS_1989_UTM_Zone_32N & Germany \\
\hline BW_1851-305-1 & $1851 / 305-1$ & GWM 1 TIEF, DOSSENHEIM & 473896 & 5477008 & ETRS_1989_UTM_Zone_32N & Germany \\
\hline BW_710-256-3 & $710 / 256-3$ & ALTLUSSHEIM M & 463216 & 5461626 & ETRS_1989_UTM_Zone_32N & Germany \\
\hline BW_765-306-3 & $765 / 306-3$ & KETSCH SCHLEIWIESE T & 463711 & 5467331 & ETRS_1989_UTM_Zone_32N & Germany \\
\hline BW_781-304-2 & $781 / 304-2$ & GWM Tief, Heddesheim & 473915 & 5486080 & ETRS_1989_UTM_Zone_32N & Germany \\
\hline HE_11874 & 11874 & RUESSELSHEIM & 462420 & 5537724 & ETRS_1989_UTM_Zone_32N & Germany \\
\hline HE_12905 & 12905 & GROSS-ROHRHEIM & 461300 & 5508386 & ETRS_1989_UTM_Zone_32N & Germany \\
\hline HE_12946 & 12946 & FEHLHEIM & 468827 & 5505887 & ETRS_1989_UTM_Zone_32N & Germany \\
\hline HE_13490 & 13490 & HOFHEIM & 458537 & 5500283 & ETRS_1989_UTM_Zone_32N & Germany \\
\hline HE_13518 & 13518 & LAMPERTHEIM & 468337 & 5494261 & ETRS_1989_UTM_Zone_32N & Germany \\
\hline RP_2375118200 & 2375118200 & 1174 II Neupotz & 451064 & 5439203 & ETRS_1989_UTM_Zone_32N & Germany \\
\hline RP_2375283300 & 2375283300 & 1273 III Leimersheim & 452633 & 5439833 & ETRS_1989_UTM_Zone_32N & Germany \\
\hline
\end{tabular}


Table S2: Results of bayesian hyperparameter optimization (NARX models).

\begin{tabular}{|c|c|c|c|c|c|c|c|c|c|c|c|}
\hline & NARY & & puts & & & & Model & Paramete & & & \\
\hline & NARX & $\mathrm{rH}$ & r 1 & $T \sin$ & Hidden size & ID P & ID rH & ID T & ID Tsin I & ID $G_{W L}$ t-1 FD & \\
\hline & BW_1-115-8 & 0 & 1 & 1 & 1 & 52 & - & 3 & 29 & - & 47 \\
\hline & BW_-103-261-4 & 1 & 1 & 0 & 18 & 12 & 3 & 52 & - & - & 39 \\
\hline & BW_104-114-5 & 0 & 1 & 1 & 14 & 8 & - & 50 & 4 & - & 36 \\
\hline & BW_104-307-0 & 0 & 1 & 1 & 20 & 50 & - & 52 & 51 & - & 11 \\
\hline & BW_138-019-0 & 0 & 0 & 1 & 19 & 24 & - & - & 50 & - & 50 \\
\hline & BW_157-260-8 & 1 & 1 & 0 & 14 & 21 & 52 & 52 & - & - & 23 \\
\hline & BW_1851-305-1 & 1 & 1 & 1 & 10 & 40 & 12 & 34 & 13 & - & 34 \\
\hline $\bar{\pi}$ & BW_710-256-3 & 0 & 0 & 1 & 18 & 12 & - & - & 46 & - & 9 \\
\hline สู & BW_765-306-3 & 1 & 0 & 1 & 17 & 18 & 51 & - & 46 & - & 33 \\
\hline$\Phi$ & BW_781-304-2 & 1 & 1 & 0 & 10 & 41 & 40 & 52 & - & - & 38 \\
\hline & HE_11874 & 0 & 0 & 1 & 18 & 37 & - & - & 49 & - & 47 \\
\hline & HE_12905 & 1 & 0 & 1 & 9 & 5 & 2 & - & 17 & - & 4 \\
\hline & HE_12946 & 1 & 0 & 0 & 7 & 44 & 47 & - & - & - & 45 \\
\hline & HE_13490 & 1 & 0 & 0 & 17 & 48 & 48 & - & - & - & 21 \\
\hline & HE_13518 & 1 & 0 & 0 & 20 & 52 & 32 & - & - & - & 10 \\
\hline & RP_2375118200 & 1 & 1 & 1 & 12 & 42 & 47 & 35 & 1 & - & 26 \\
\hline & $\mathrm{RP}^{-} 2375283300$ & 1 & 1 & 1 & 8 & 11 & 21 & 47 & 25 & - & 4 \\
\hline & BW_1-115-8 & 0 & 1 & 0 & 1 & 13 & - & 19 & - & 44 & 8 \\
\hline & BW_103-261-4 & 1 & 0 & 0 & 1 & 17 & 19 & - & - & 50 & 8 \\
\hline & BW_104-114-5 & 1 & 0 & 1 & 1 & 3 & 44 & - & 30 & 13 & 2 \\
\hline & BW_104-307-0 & 0 & 0 & 1 & 3 & 28 & - & - & 11 & 14 & 10 \\
\hline & BW_138-019-0 & 0 & 1 & 1 & 4 & 2 & - & 5 & 48 & 8 & 3 \\
\hline & BW_157-260-8 & 0 & 1 & 1 & 1 & 19 & - & 18 & 7 & 3 & \\
\hline 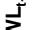 & BW_1851-305-1 & 0 & 1 & 0 & 1 & 12 & - & 26 & - & 5 & 14 \\
\hline$\frac{3}{0}$ & BW_710-256-3 & 0 & 0 & 1 & 2 & 3 & - & - & 35 & 5 & 3 \\
\hline$\frac{\sigma}{\sigma}$ & BW_765-306-3 & 0 & 0 & 0 & 3 & 2 & - & - & - & 45 & 2 \\
\hline בั่ & BW_781-304-2 & 1 & 1 & 0 & 3 & 6 & 8 & 10 & - & 28 & \\
\hline $\bar{\Phi}_{0}^{\circ}$ & HE_11874 & 0 & 1 & 1 & 4 & 4 & - & 13 & 32 & 51 & 11 \\
\hline & HE_12905 & 1 & 1 & 0 & 1 & 18 & 7 & 21 & - & 30 & 1 \\
\hline & HE_12946 & 1 & 0 & 0 & 1 & 2 & 16 & - & - & 3 & 12 \\
\hline & HE_13490 & 0 & 1 & 1 & 8 & 3 & - & 6 & 44 & 28 & \\
\hline & HE_13518 & 1 & 1 & 1 & 1 & 4 & 22 & 10 & 14 & 1 & \\
\hline & RP_2375118200 & 0 & 1 & 1 & 7 & 7 & - & 28 & 38 & 7 & \\
\hline & RP_2375283300 & 0 & 0 & 1 & 2 & 3 & - & - & 35 & 5 & 3 \\
\hline & BW_1-115-8 & 0 & 1 & 0 & 1 & 2 & - & 9 & - & - & 8 \\
\hline & BW_103-261-4 & 0 & 1 & 1 & 17 & 1 & - & 1 & 4 & - & \\
\hline & BW_104-114-5 & 0 & 1 & 1 & 1 & 4 & - & 8 & 8 & - & 4 \\
\hline & BW_104-307-0 & 1 & 0 & 0 & 17 & 11 & 11 & - & - & - & 5 \\
\hline & BW_138-019-0 & 0 & 0 & 0 & 1 & 8 & - & - & - & - & 2 \\
\hline & BW_157-260-8 & 1 & 1 & 0 & 20 & 10 & 2 & 1 & - & - & 3 \\
\hline & BW_1851-305-1 & 0 & 0 & 0 & 10 & 11 & - & - & - & - & \\
\hline $\bar{\delta}$ & BW_710-256-3 & 1 & 0 & 0 & 20 & 12 & 4 & - & - & - & 1 \\
\hline ปู & BW_765-306-3 & 0 & 0 & 1 & 20 & 4 & - & - & 7 & - & 3 \\
\hline ֻ & BW_781-304-2 & 1 & 0 & 0 & 17 & 11 & 11 & - & - & - & 5 \\
\hline & HE_-11874 & 1 & 1 & 1 & 8 & 10 & 8 & 12 & 5 & - & 12 \\
\hline & HE_12905 & 1 & 1 & 0 & 12 & 6 & 6 & 1 & - & - & 8 \\
\hline & HE_12946 & 1 & 0 & 0 & 20 & 12 & 7 & - & - & - & 5 \\
\hline & HE_13490 & 1 & 1 & 0 & 3 & 4 & 4 & 1 & - & - & 3 \\
\hline & HE_13518 & 1 & 1 & 0 & 18 & 10 & 7 & 12 & - & - & 12 \\
\hline & RP_2375118200 & 1 & 1 & 1 & 2 & 10 & 11 & 4 & 12 & - & 12 \\
\hline & RP 2375283300 & 1 & 0 & 0 & 4 & 8 & 4 & - & - & - & 3 \\
\hline & BW_1-115-8 & 0 & 1 & 1 & 1 & 3 & - & 12 & 1 & 11 & 8 \\
\hline & BW_103-261-4 & 1 & 0 & 1 & 7 & 6 & 11 & - & 3 & 8 & 2 \\
\hline & BW_104-114-5 & 1 & 1 & 1 & 2 & 6 & 2 & 12 & 3 & 11 & 6 \\
\hline & BW_104-307-0 & 0 & 1 & 0 & 19 & 12 & - & 1 & - & 12 & 2 \\
\hline & BW_138-019-0 & 1 & 0 & 0 & 8 & 9 & 2 & - & - & 2 & 6 \\
\hline & BW_-157-260-8 & 1 & 1 & 1 & 2 & 2 & 3 & 11 & 4 & 1 & 1 \\
\hline & BW_1851-305-1 & 1 & 1 & 0 & 17 & 10 & 10 & 5 & - & 2 & 6 \\
\hline 5 & BW_710-256-3 & 0 & 1 & 1 & 1 & 3 & - & 6 & 12 & 3 & 11 \\
\hline d & BW_765-306-3 & 0 & 1 & 1 & 5 & 3 & - & 3 & 7 & 5 & 9 \\
\hline ูู & BW_781-304-2 & 1 & 0 & 0 & 20 & 9 & 3 & - & - & 1 & 7 \\
\hline \% & HE_11874 & 1 & 1 & 1 & 20 & 3 & 10 & 11 & 8 & 10 & 8 \\
\hline & HE_12905 & 0 & 1 & 1 & 4 & 8 & - & 9 & 7 & 8 & 9 \\
\hline & HE_12946 & 0 & 0 & 0 & 17 & 1 & - & - & - & 11 & 12 \\
\hline & HE_13490 & 1 & 0 & 1 & 6 & 2 & 2 & - & 12 & 6 & 9 \\
\hline & HE_13518 & 0 & 1 & 1 & 1 & 12 & - & 1 & 7 & 10 & 12 \\
\hline & RP_2375118200 & 0 & 1 & 0 & 2 & 7 & - & 11 & - & 10 & 2 \\
\hline & $\operatorname{RP}^{-} 2375283300$ & 1 & 0 & 0 & 1 & 12 & 6 & - & - & 2 & \\
\hline
\end{tabular}


Table S3: Results of bayesian hyperparameter optimization (LSTM models).

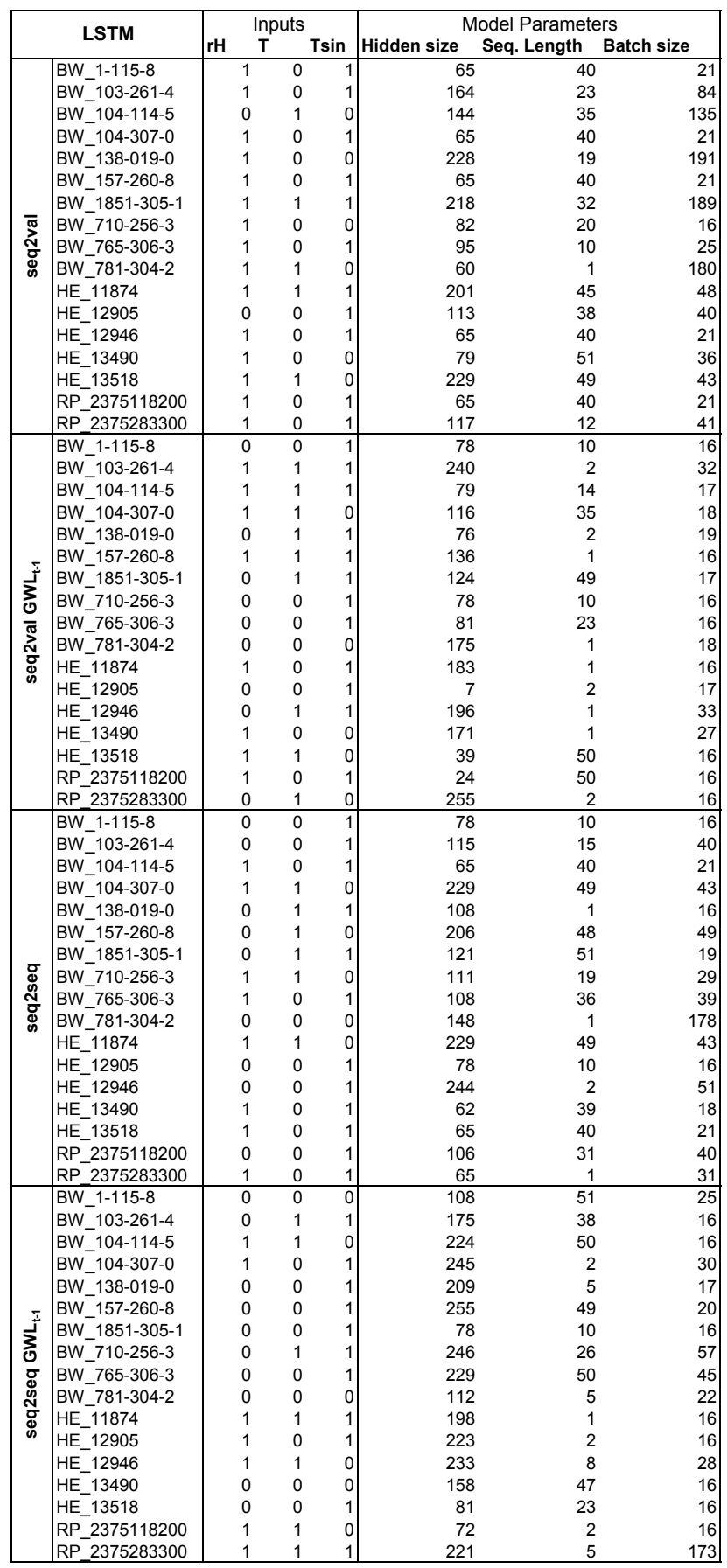


Table S4: Results of bayesian hyperparameter optimization (CNN models).

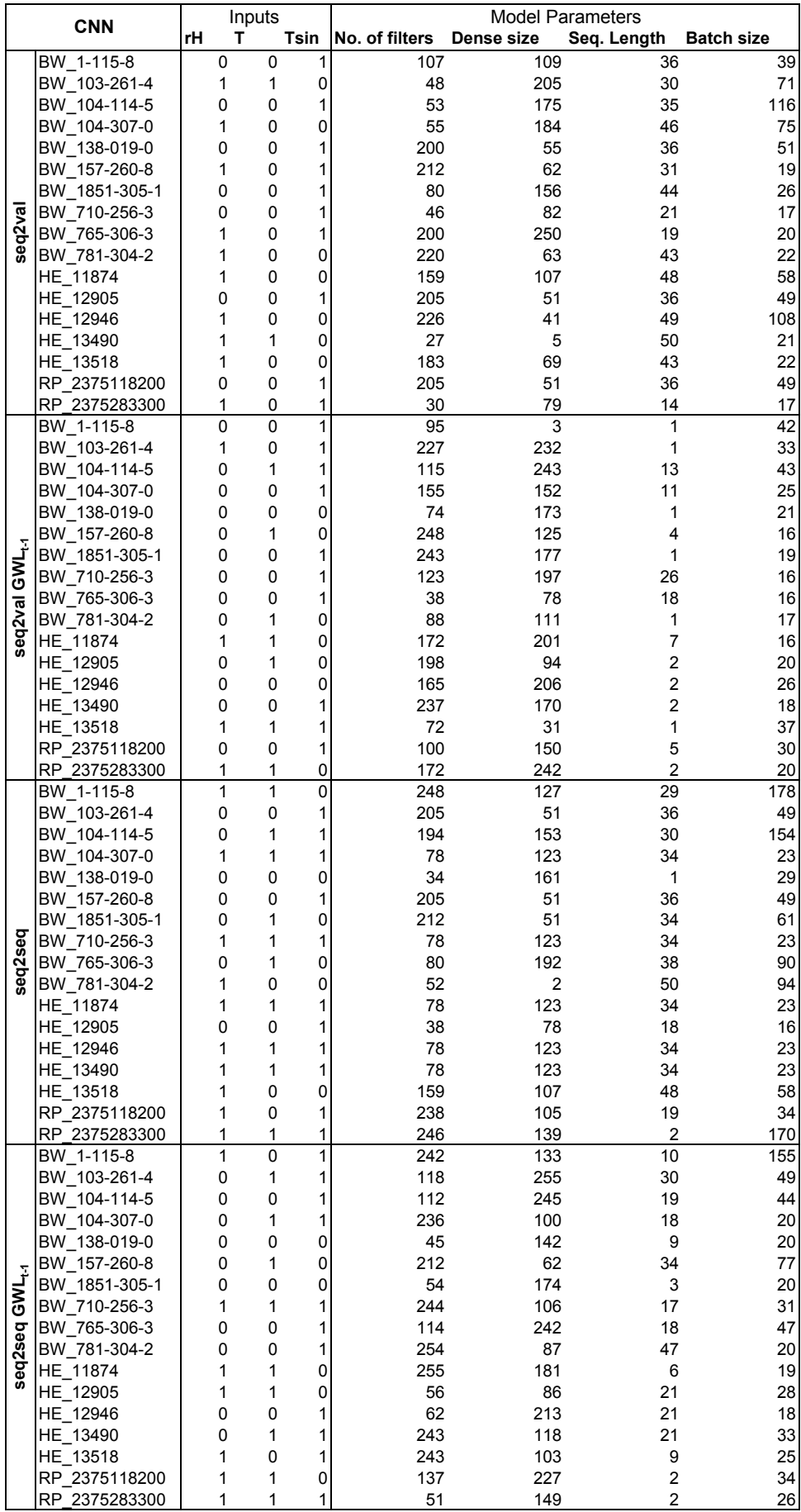


Table S5: Forecasting results of all sequence-to-value models.

\begin{tabular}{|c|c|c|c|c|c|c|c|c|c|c|c|c|c|c|c|}
\hline & & & & & Seq2Va & & & & & & Seq2 & al (GW & $t-1)$ & & \\
\hline \multirow{18}{*}{$\begin{array}{l}\underset{\mathfrak{c}}{\mathbf{\alpha}} \\
\mathbf{z}\end{array}$} & ID & NSE & R2 & RMSE & rRMSE & Bias & rBias & PI & NSE & R2 & RMSE & rRMSE & Bias & rBias & PI \\
\hline & BW_1-115-8 & 0.68 & 0.83 & 0.17 & 11.85 & -0.13 & -8.86 & -8.55 & 0.86 & 0.85 & 0.11 & 7.89 & -0.04 & -2.82 & -3.23 \\
\hline & BW_103-261-4 & 0.67 & 0.77 & 0.21 & 8.45 & -0.13 & -5.24 & -5.42 & 0.93 & 0.91 & 0.10 & 3.89 & 0.00 & -0.15 & -0.36 \\
\hline & BW_104-114-5 & 0.84 & 0.87 & 0.17 & 5.80 & -0.08 & -2.58 & -0.33 & 0.89 & 0.89 & 0.14 & 4.87 & -0.01 & -0.19 & 0.06 \\
\hline & BW_104-307-0 & -0.68 & 0.71 & 0.25 & 13.88 & -0.23 & -12.55 & -85.04 & 0.96 & 0.96 & 0.04 & 2.08 & 0.00 & -0.17 & -0.92 \\
\hline & BW_138-019-0 & 0.06 & 0.13 & 0.11 & 5.46 & -0.05 & -2.58 & -0.81 & 0.41 & 0.31 & 0.08 & 4.33 & -0.01 & -0.45 & -0.14 \\
\hline & BW_157-260-8 & 0.78 & 0.90 & 0.13 & 8.11 & 0.11 & 6.45 & -5.86 & 0.96 & 0.95 & 0.06 & 3.43 & 0.00 & -0.08 & -0.23 \\
\hline & BW_1851-305-1 & 0.75 & 0.71 & 0.15 & 8.40 & 0.05 & 2.61 & -3.34 & 0.90 & 0.84 & 0.09 & 5.29 & 0.02 & 1.03 & -0.72 \\
\hline & BW_710-256-3 & 0.42 & 0.62 & 0.31 & 9.41 & -0.17 & -5.36 & -4.65 & 0.84 & 0.84 & 0.16 & 4.91 & -0.01 & -0.34 & -0.54 \\
\hline & BW_765-306-3 & 0.30 & 0.40 & 0.53 & 13.58 & -0.24 & -6.12 & -2.27 & 0.67 & 0.66 & 0.37 & 9.32 & -0.06 & -1.60 & -0.54 \\
\hline & BW_781-304-2 & 0.90 & 0.60 & 0.18 & 7.12 & -0.10 & -4.21 & -26.70 & 0.99 & 0.96 & 0.04 & 1.81 & 0.00 & -0.06 & -0.80 \\
\hline & HE_11874 & 0.35 & 0.75 & 0.24 & 13.42 & -0.17 & -9.38 & -55.95 & 0.99 & 0.99 & 0.03 & 1.45 & -0.01 & -0.40 & 0.33 \\
\hline & HE_12905 & 0.26 & 0.67 & 0.40 & 11.86 & -0.31 & -9.28 & -14.92 & 0.92 & 0.90 & 0.14 & 4.01 & -0.03 & -0.87 & -0.82 \\
\hline & HE_12946 & 0.86 & 0.18 & 0.25 & 8.67 & 0.08 & 2.88 & -42.39 & 1.00 & 0.93 & 0.04 & 1.52 & 0.00 & -0.08 & -0.33 \\
\hline & HE_13490 & 0.56 & 0.75 & 0.24 & 11.89 & -0.22 & -10.47 & -28.53 & 0.98 & 0.96 & 0.05 & 2.43 & -0.01 & -0.26 & -0.23 \\
\hline & HE_13518 & 0.76 & 0.87 & 0.20 & 9.05 & -0.19 & -8.38 & -53.81 & 0.99 & 0.98 & 0.03 & 1.47 & 0.00 & 0.09 & -0.46 \\
\hline & RP_2375118200 & 0.55 & 0.78 & 0.17 & 14.45 & -0.15 & -12.17 & -14.48 & 0.95 & 0.93 & 0.06 & 4.64 & -0.02 & -1.57 & -0.59 \\
\hline & RP_2375283300 & 0.68 & 0.66 & 0.20 & 9.33 & -0.11 & -5.12 & -1.77 & 0.81 & 0.74 & 0.16 & 7.27 & -0.06 & -2.56 & -0.68 \\
\hline \multirow{18}{*}{$\underset{\Xi}{\underline{\Xi}}$} & ID & NSE & R2 & RMSE & rRMSE & Bias & rBias & $\mathbf{P I}$ & NSE & R2 & RMSE & rRMSE & Bias & rBias & PI \\
\hline & BW_1-115-8 & 0.76 & 0.83 & 0.15 & 10.36 & -0.10 & -6.63 & -6.24 & 0.87 & 0.86 & 0.11 & 7.63 & -0.04 & -2.48 & -2.93 \\
\hline & BW_103-261-4 & 0.61 & 0.72 & 0.23 & 9.21 & -0.15 & -6.07 & -6.66 & 0.91 & 0.89 & 0.11 & 4.32 & -0.01 & -0.44 & -0.69 \\
\hline & BW_104-114-5 & 0.61 & 0.71 & 0.26 & 9.02 & -0.14 & -4.90 & -2.24 & 0.88 & 0.88 & 0.15 & 4.96 & -0.01 & -0.35 & 0.02 \\
\hline & BW_104-307-0 & 0.49 & 0.50 & 0.14 & 7.58 & -0.03 & -1.39 & -24.89 & 0.95 & 0.95 & 0.04 & 2.34 & -0.01 & -0.61 & -1.48 \\
\hline & BW_138-019-0 & -0.01 & 0.00 & 0.11 & 5.65 & -0.04 & -2.24 & -0.95 & 0.37 & 0.29 & 0.09 & 4.47 & -0.02 & -0.78 & -0.22 \\
\hline & BW_157-260-8 & 0.81 & 0.84 & 0.12 & 7.39 & 0.04 & 2.36 & -4.68 & 0.96 & 0.95 & 0.06 & 3.54 & 0.00 & 0.08 & -0.30 \\
\hline & BW_1851-305-1 & -0.13 & 0.15 & 0.32 & 17.93 & 0.24 & 13.56 & -18.79 & 0.90 & 0.83 & 0.09 & 5.29 & 0.01 & 0.61 & -0.72 \\
\hline & BW_710-256-3 & 0.48 & 0.57 & 0.29 & 8.94 & -0.12 & -3.67 & -4.00 & 0.78 & 0.79 & 0.19 & 5.75 & -0.04 & -1.28 & -1.07 \\
\hline & BW_765-306-3 & 0.41 & 0.42 & 0.49 & 12.56 & -0.15 & -3.74 & -1.78 & 0.67 & 0.66 & 0.37 & 9.44 & -0.08 & -2.16 & -0.57 \\
\hline & BW_781-304-2 & 0.11 & 0.00 & 0.53 & 21.24 & -0.48 & -19.48 & -245.79 & 0.99 & 0.95 & 0.05 & 1.89 & 0.00 & -0.06 & -0.95 \\
\hline & HE_11874 & 0.45 & 0.49 & 0.22 & 12.31 & 0.05 & 2.62 & -47.10 & 0.98 & 0.98 & 0.04 & 2.09 & 0.00 & -0.16 & -0.39 \\
\hline & HE_12905 & 0.20 & 0.62 & 0.41 & 12.27 & -0.32 & -9.54 & -15.66 & 0.88 & 0.86 & 0.16 & 4.76 & -0.03 & -0.75 & -1.51 \\
\hline & HE_12946 & 0.46 & 0.52 & 0.49 & 17.02 & -0.44 & -15.06 & -166.68 & 0.99 & 0.91 & 0.05 & 1.67 & 0.00 & 0.05 & -0.62 \\
\hline & HE_13490 & -0.33 & 0.62 & 0.42 & 20.58 & -0.40 & -19.35 & -87.98 & 0.98 & 0.94 & 0.06 & 2.68 & 0.01 & 0.27 & -0.51 \\
\hline & HE_13518 & 0.33 & 0.36 & 0.34 & 14.99 & -0.29 & -12.74 & -144.04 & 0.99 & 0.96 & 0.04 & 1.83 & 0.00 & -0.11 & -1.16 \\
\hline & RP_2375118200 & 0.63 & 0.75 & 0.16 & 13.11 & -0.12 & -10.11 & -11.33 & 0.94 & 0.91 & 0.07 & 5.47 & -0.03 & -2.40 & -1.14 \\
\hline & RP_2375283300 & 0.41 & 0.56 & 0.28 & 12.82 & -0.20 & -9.27 & -4.15 & 0.77 & 0.68 & 0.17 & 8.00 & -0.05 & -2.49 & -1.00 \\
\hline \multirow{18}{*}{ ż } & ID & NSE & R2 & RMSE & rRMSE & Bias & rBias & $\mathbf{P I}$ & NSE & R2 & RMSE & rRMSE & Bias & rBias & PI \\
\hline & BW_1-115-8 & 0.61 & 0.75 & 0.19 & 13.05 & -0.13 & -8.83 & \begin{tabular}{|c|}
-10.48 \\
\end{tabular} & 0.89 & 0.89 & 0.10 & 7.00 & -0.03 & -2.35 & -2.30 \\
\hline & BW_103-261-4 & 0.65 & 0.72 & 0.22 & 8.72 & -0.12 & -4.73 & -5.88 & 0.92 & 0.89 & 0.11 & 4.25 & 0.00 & -0.19 & -0.63 \\
\hline & BW_104-114-5 & 0.72 & 0.75 & 0.22 & 7.62 & -0.07 & -2.43 & -1.32 & 0.89 & 0.89 & 0.14 & 4.75 & -0.01 & -0.34 & 0.10 \\
\hline & BW_104-307-0 & 0.61 & 0.69 & 0.12 & 6.67 & 0.06 & 3.14 & -19.07 & 0.95 & 0.95 & 0.04 & 2.28 & -0.01 & -0.28 & -1.34 \\
\hline & BW_138-019-0 & -0.10 & 0.03 & 0.11 & 5.88 & -0.06 & -2.96 & -1.11 & 0.36 & 0.27 & 0.09 & 4.51 & 0.01 & 0.38 & -0.24 \\
\hline & BW_157-260-8 & 0.83 & 0.84 & 0.11 & 7.00 & 0.02 & 1.40 & -4.10 & 0.96 & 0.95 & 0.05 & 3.36 & 0.00 & -0.04 & -0.18 \\
\hline & BW_1851-305-1 & 0.17 & 0.27 & 0.28 & 15.38 & 0.19 & 10.87 & -13.55 & 0.90 & 0.84 & 0.09 & 5.30 & 0.02 & 1.33 & -0.73 \\
\hline & BW_710-256-3 & 0.51 & 0.65 & 0.28 & 8.61 & -0.11 & -3.23 & -3.64 & 0.79 & 0.79 & 0.18 & 5.67 & -0.03 & -0.90 & -1.01 \\
\hline & BW_765-306-3 & 0.47 & 0.46 & 0.46 & 11.85 & -0.10 & -2.44 & -1.47 & 0.70 & 0.69 & 0.35 & 8.98 & -0.07 & -1.72 & -0.42 \\
\hline & BW_781-304-2 & 0.30 & 0.62 & 0.47 & 18.84 & -0.44 & -17.92 & -193.19 & 0.99 & 0.95 & 0.05 & 1.99 & 0.01 & 0.22 & -1.17 \\
\hline & HE_11874 & 0.59 & 0.63 & 0.19 & 10.66 & 0.06 & 3.29 & -35.09 & 0.99 & 0.99 & 0.03 & 1.75 & -0.01 & -0.31 & 0.03 \\
\hline & HE_12905 & 0.06 & 0.49 & 0.45 & 13.33 & -0.33 & -9.78 & -18.65 & 0.92 & 0.90 & 0.13 & 3.96 & -0.03 & -0.78 & -0.73 \\
\hline & HE_12946 & 0.26 & 0.43 & 0.58 & 19.97 & -0.56 & -19.18 & -229.89 & 0.99 & 0.90 & 0.05 & 1.76 & 0.00 & -0.04 & -0.79 \\
\hline & HE_13490 & 0.82 & 0.71 & 0.16 & 7.55 & -0.09 & -4.59 & -10.99 & 0.98 & 0.95 & 0.05 & 2.58 & 0.00 & -0.01 & -0.40 \\
\hline & HE_13518 & 0.75 & 0.62 & 0.20 & 9.05 & -0.16 & -6.89 & -51.88 & 0.99 & 0.96 & 0.04 & 1.97 & 0.01 & 0.39 & -1.49 \\
\hline & RP_2375118200 & 0.12 & 0.79 & 0.24 & 20.23 & -0.22 & -18.58 & -28.33 & 0.95 & 0.93 & 0.06 & 4.75 & -0.02 & -1.92 & -0.62 \\
\hline & RP_2375283 & 0.49 & 0.59 & 0.26 & 11.92 & -0.18 & -8.24 & -3.46 & 0.80 & 0.74 & 0.16 & 7.37 & -0.06 & -2.63 & -0.70 \\
\hline
\end{tabular}


Table S6: Forecasting results of all sequence-to-sequence models.

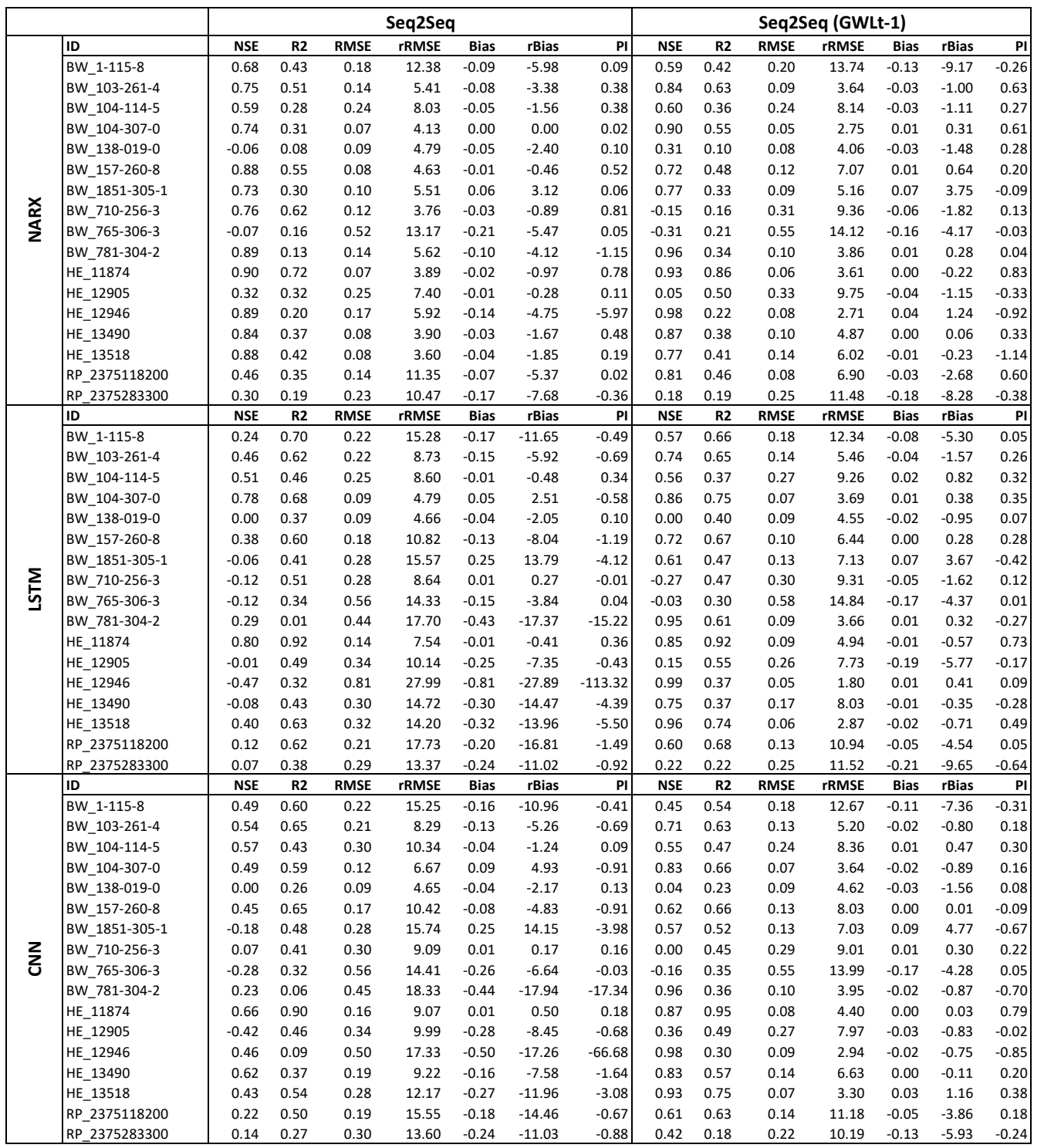



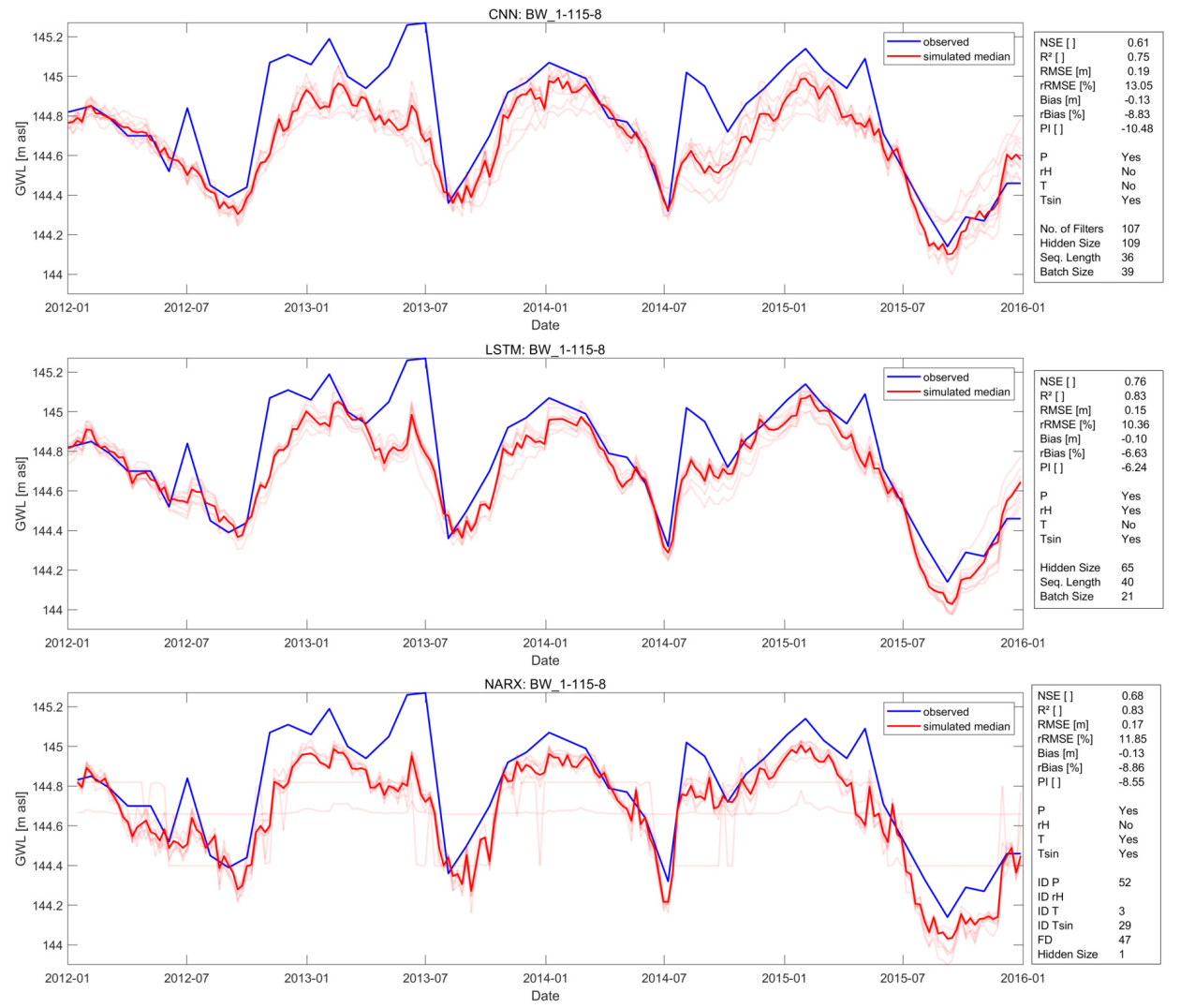

Figure S1: Seq2Val test results for well BW_1-115-8 

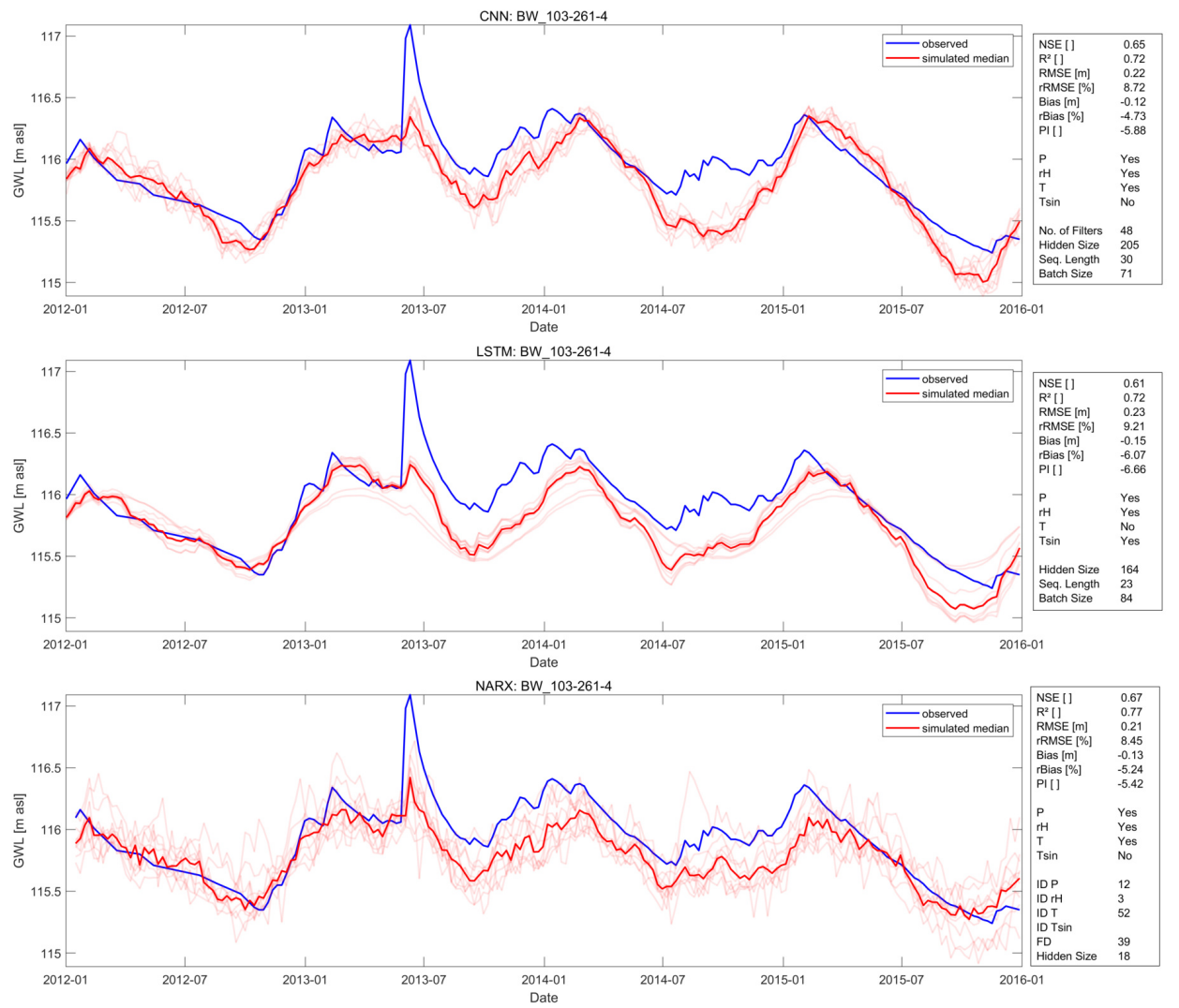

Figure S2: Seq2Val test results for well BW_103-261-4 

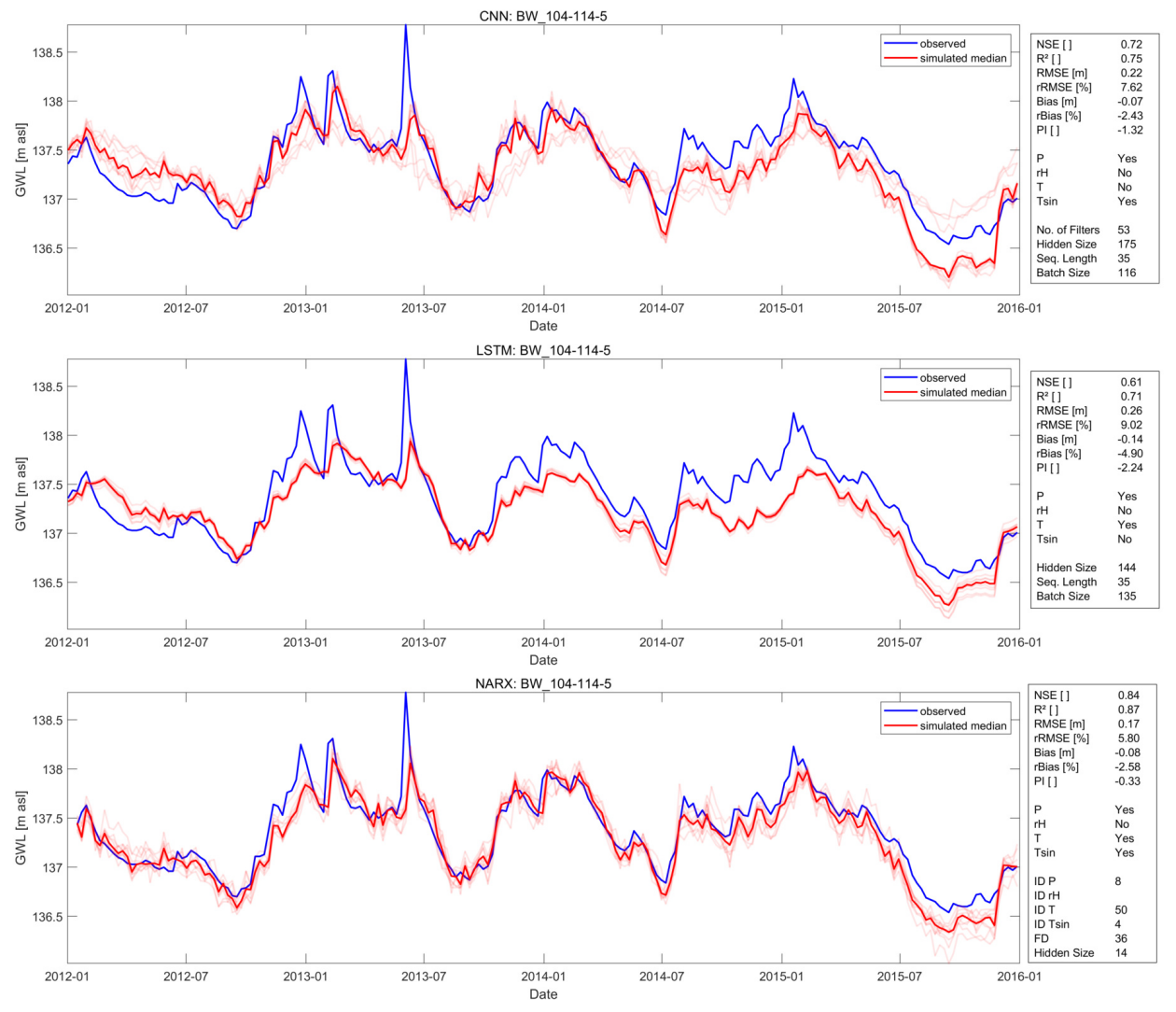

Figure S3: Seq2Val test results for well BW_104-114-5 

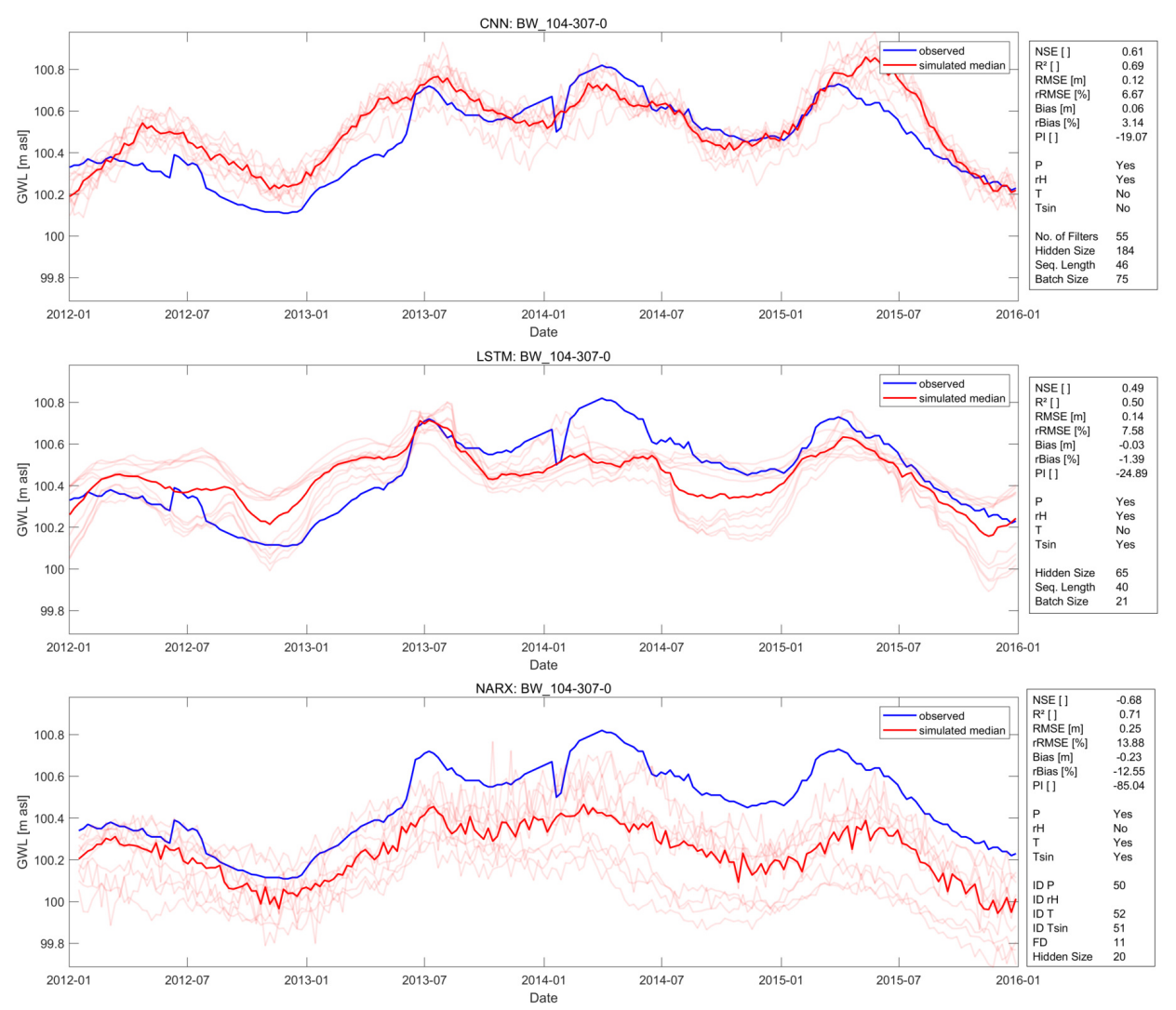

Figure S4: Seq2Val test results for well BW_104-307-0 

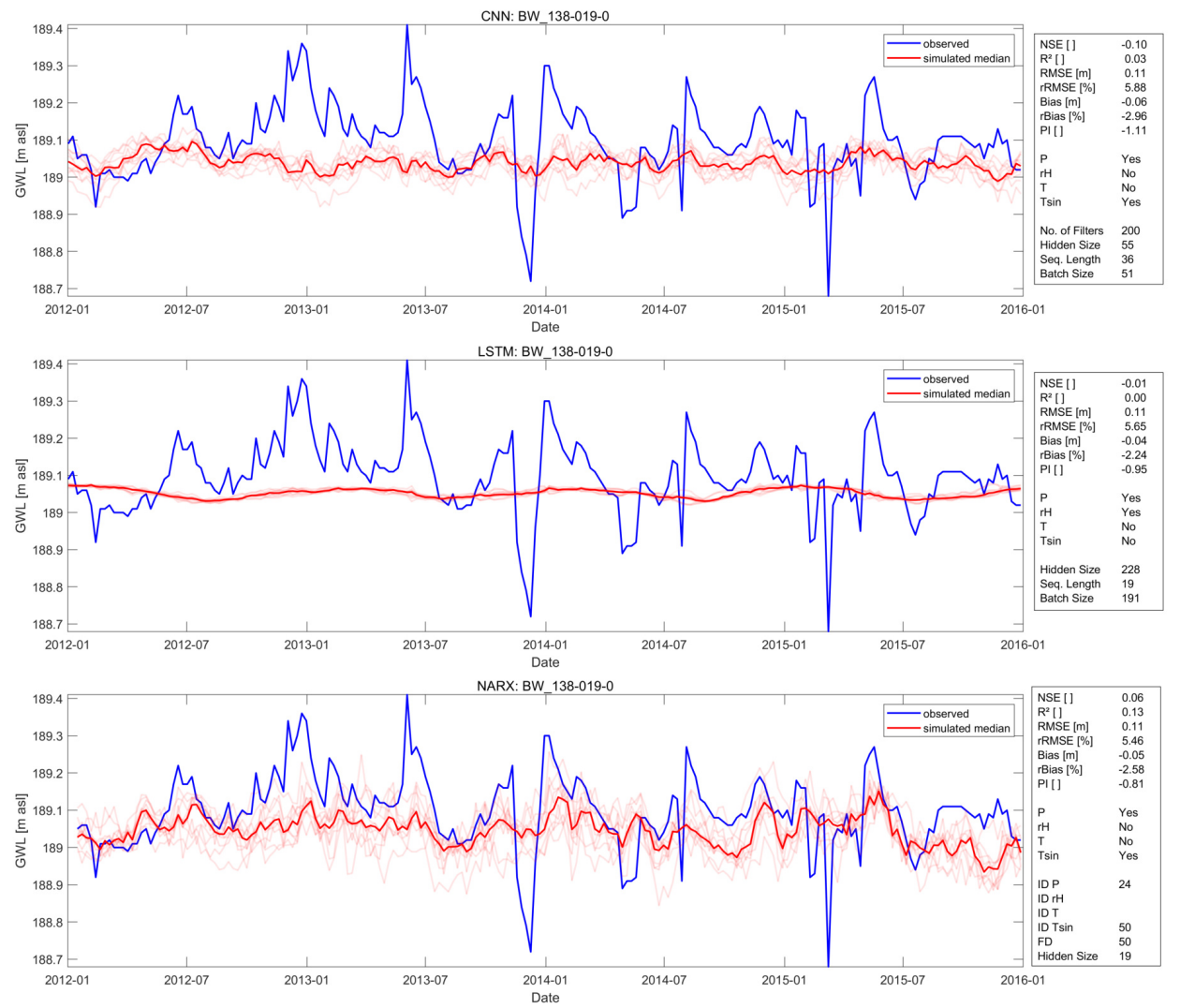

Figure S5: Seq2Val test results for well BW_138-019-0 

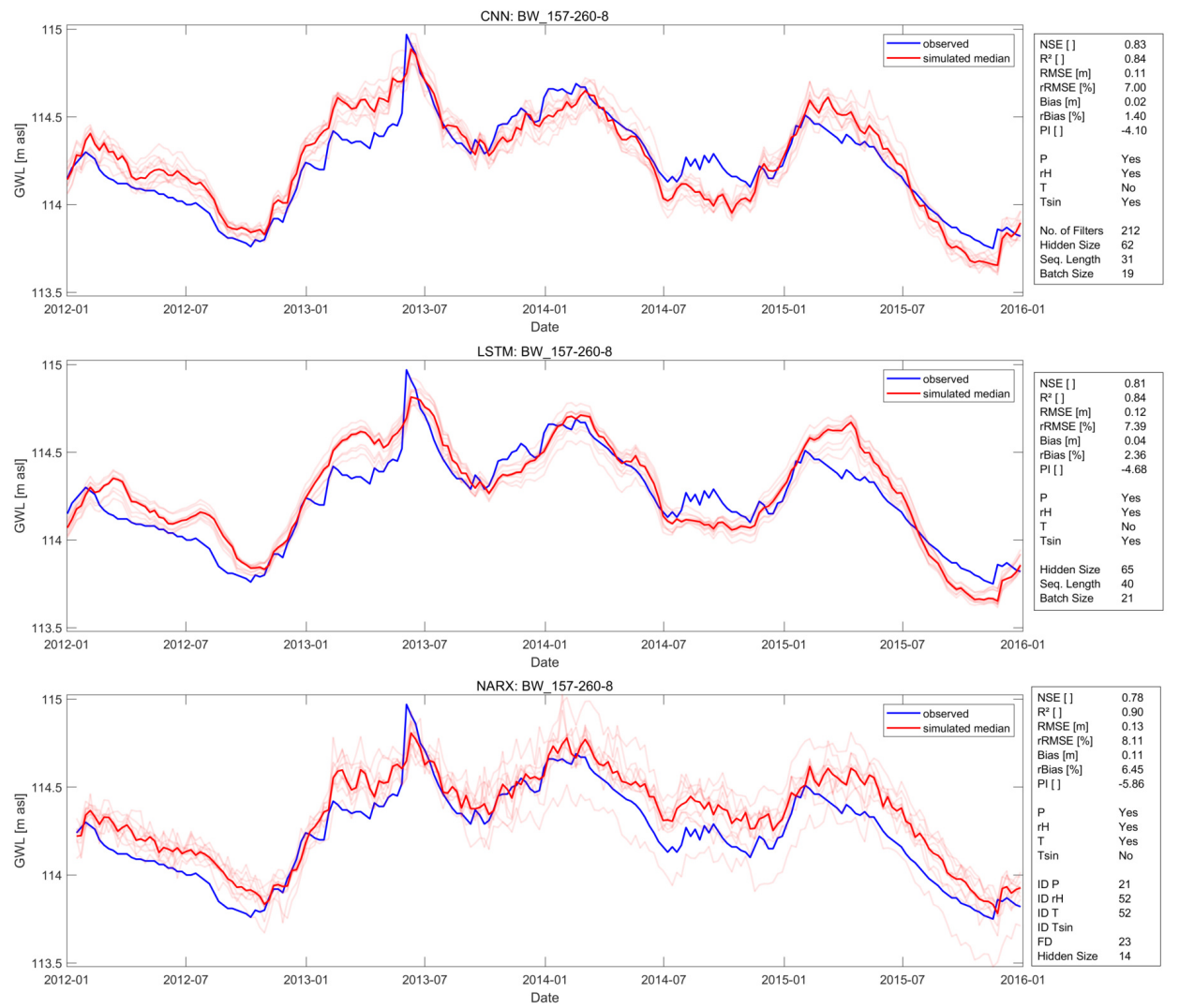

Figure S6: Seq2Val test results for well BW_157-260-8 

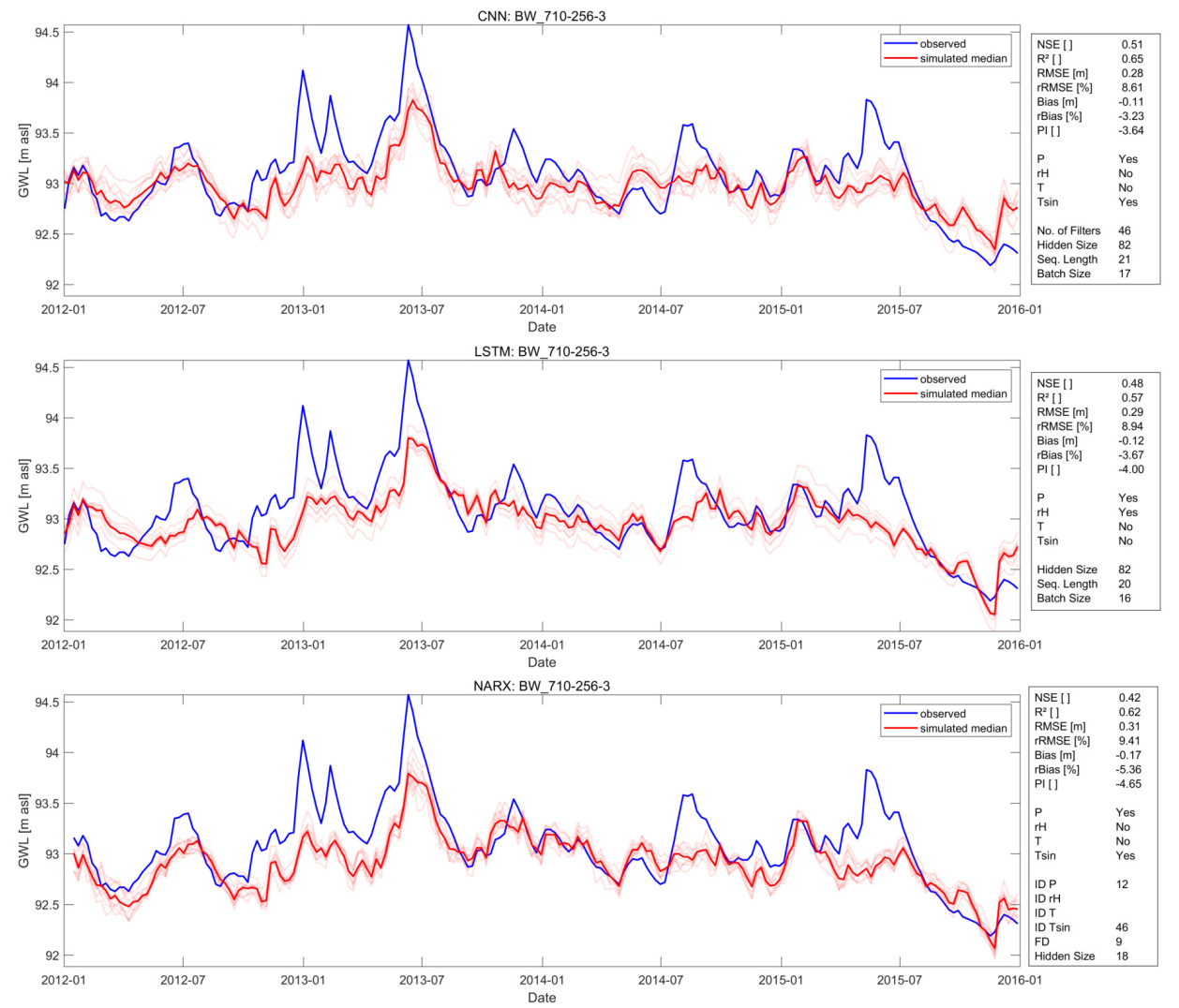

Figure S7: Seq2Val test results for well BW_710-256-3 


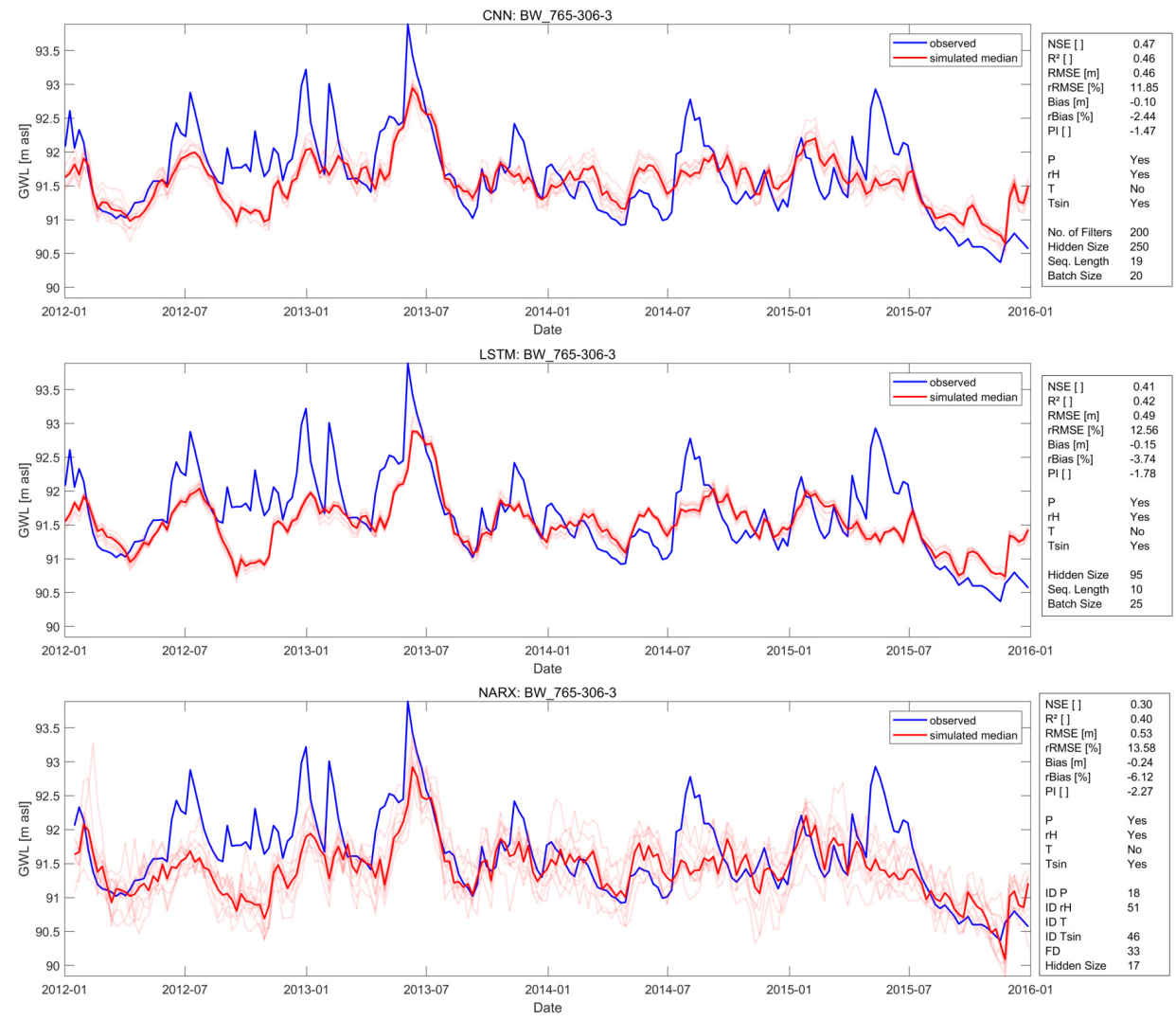

Figure S8: Seq2Val test results for well BW_765-306-3 

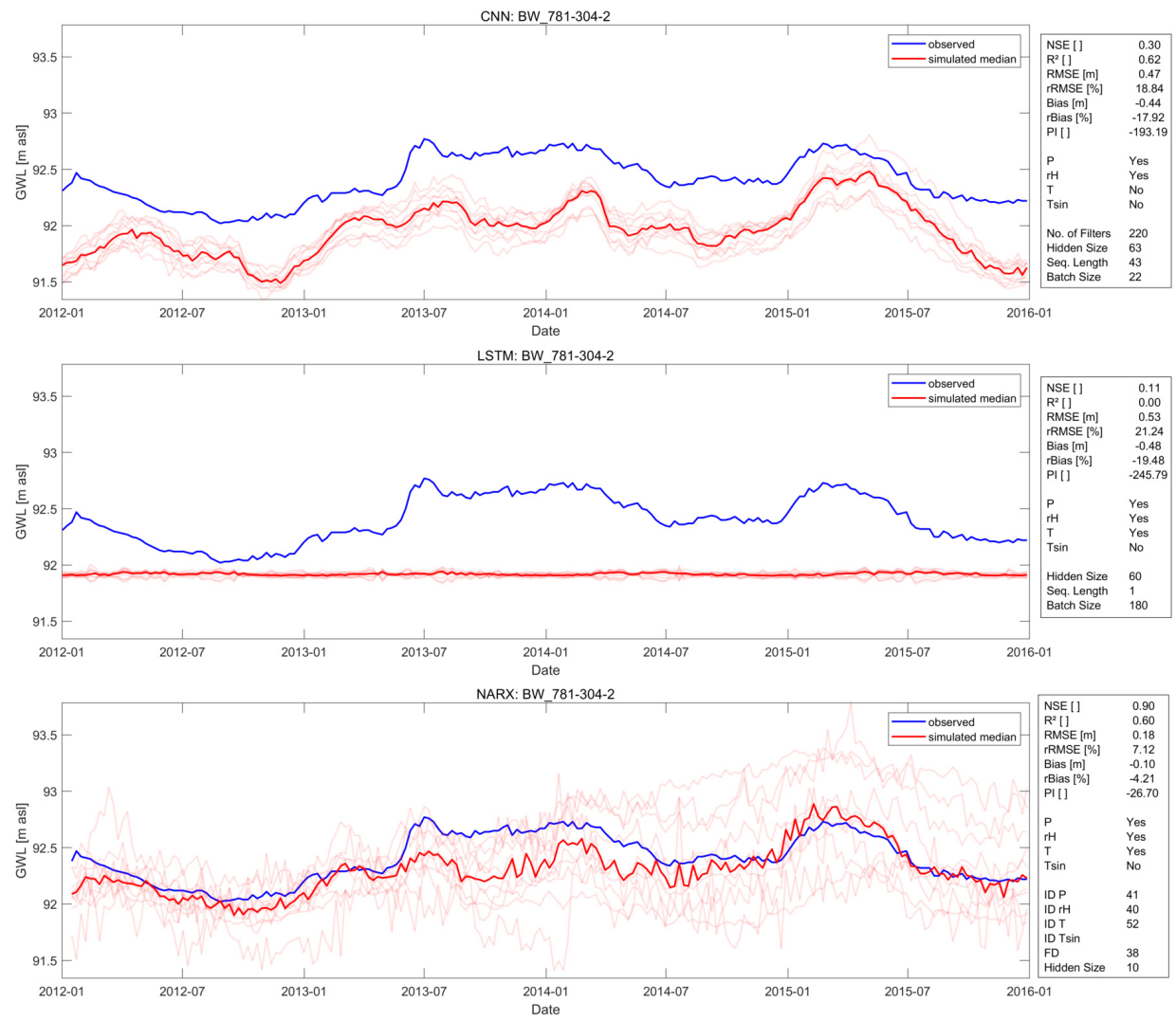

Figure S9: Seq2Val test results for well BW_781-304-2 

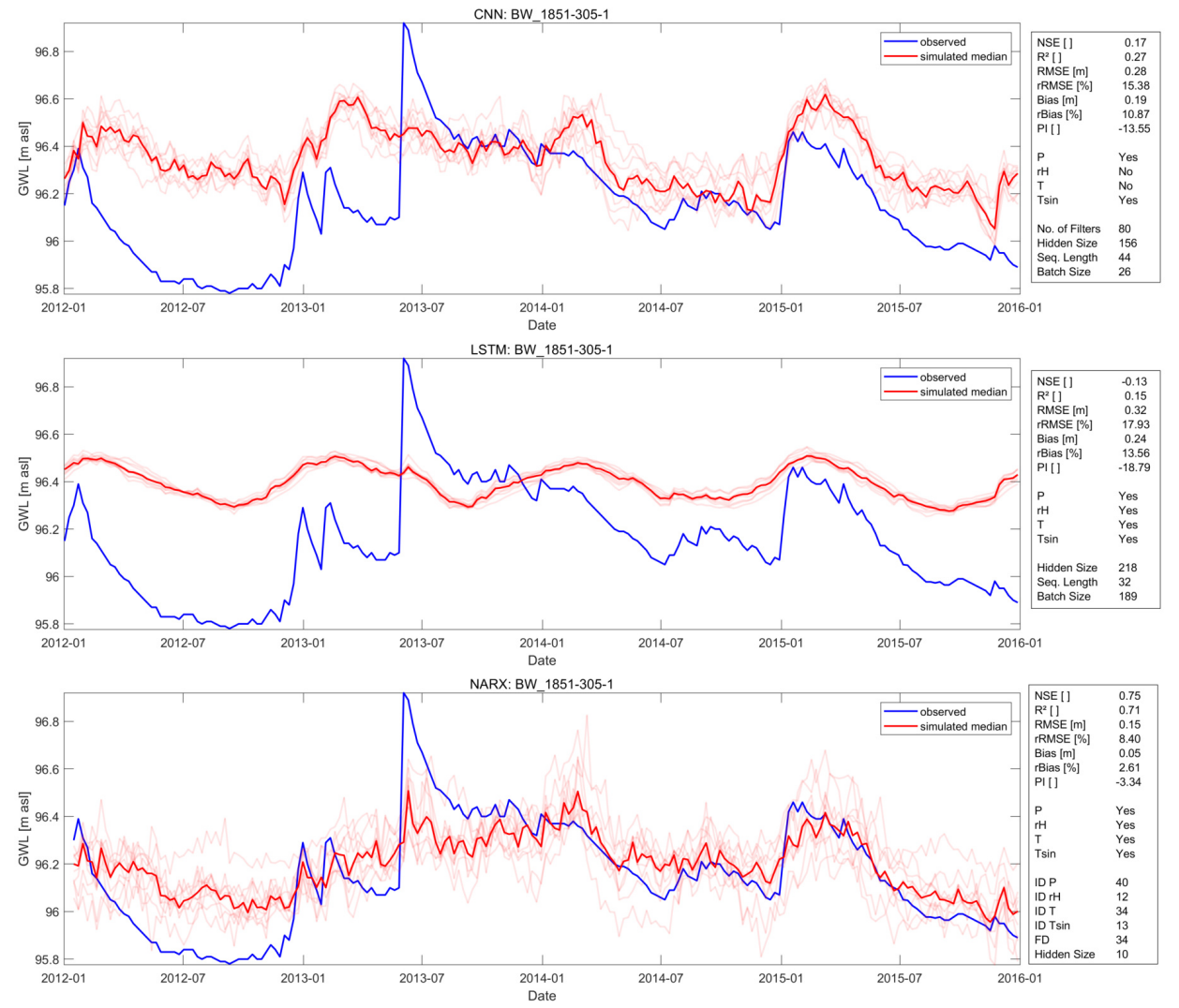

Figure S10: Seq2Val test results for well BW_1851-305-1 

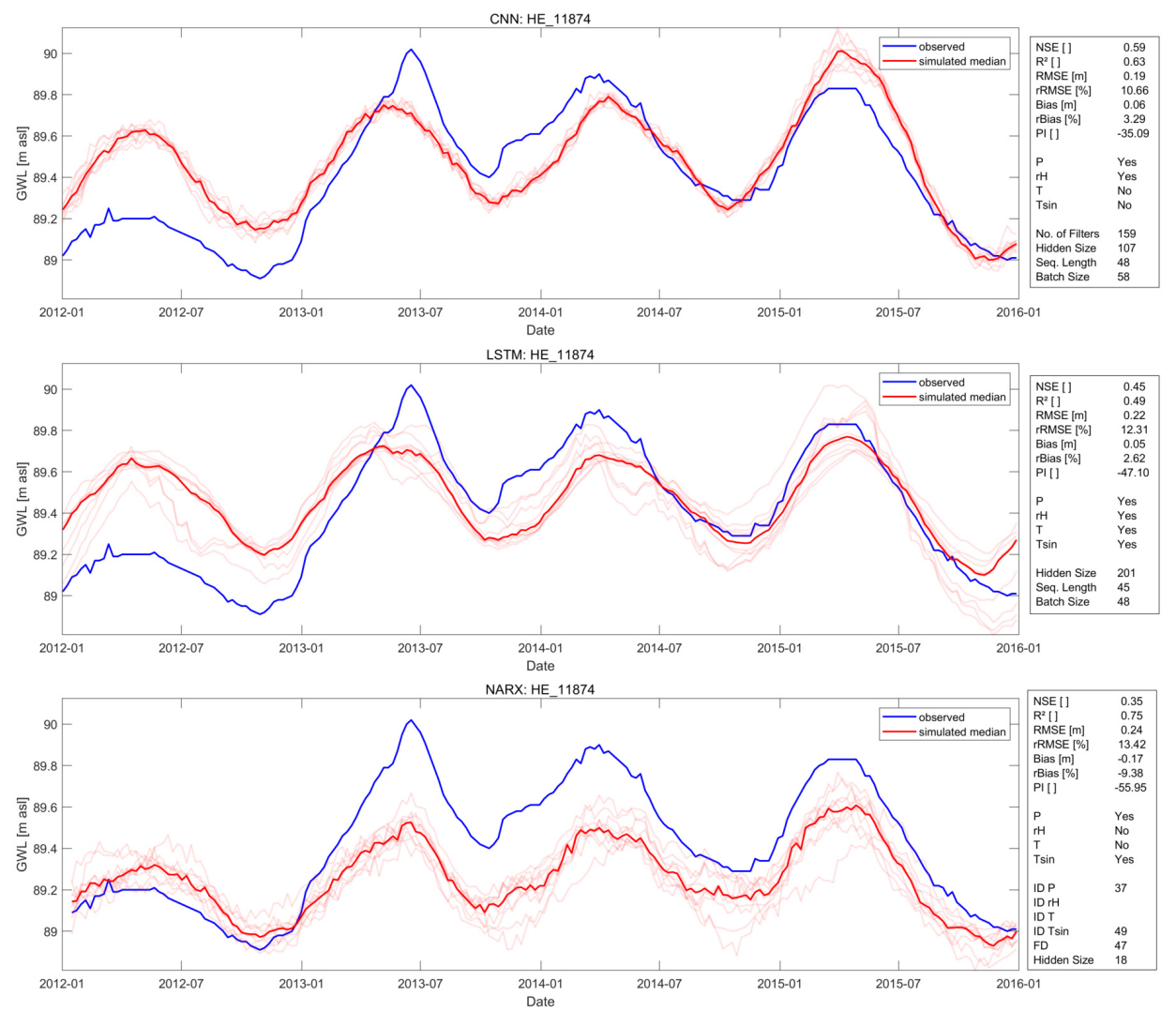

Figure S11: Seq2Val test results for well HE_11874 

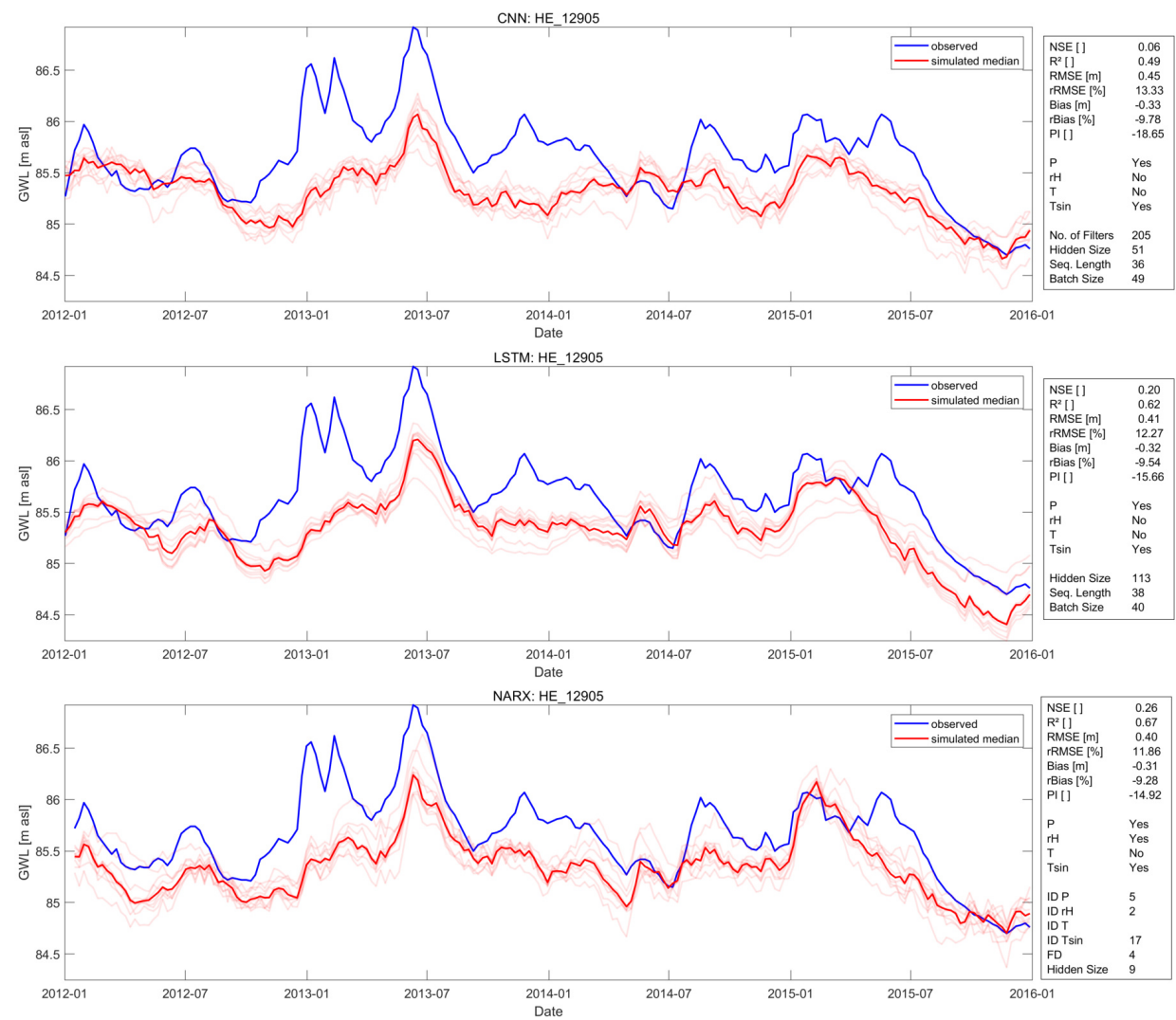

Figure S12: Seq2Val test results for well HE_12905 

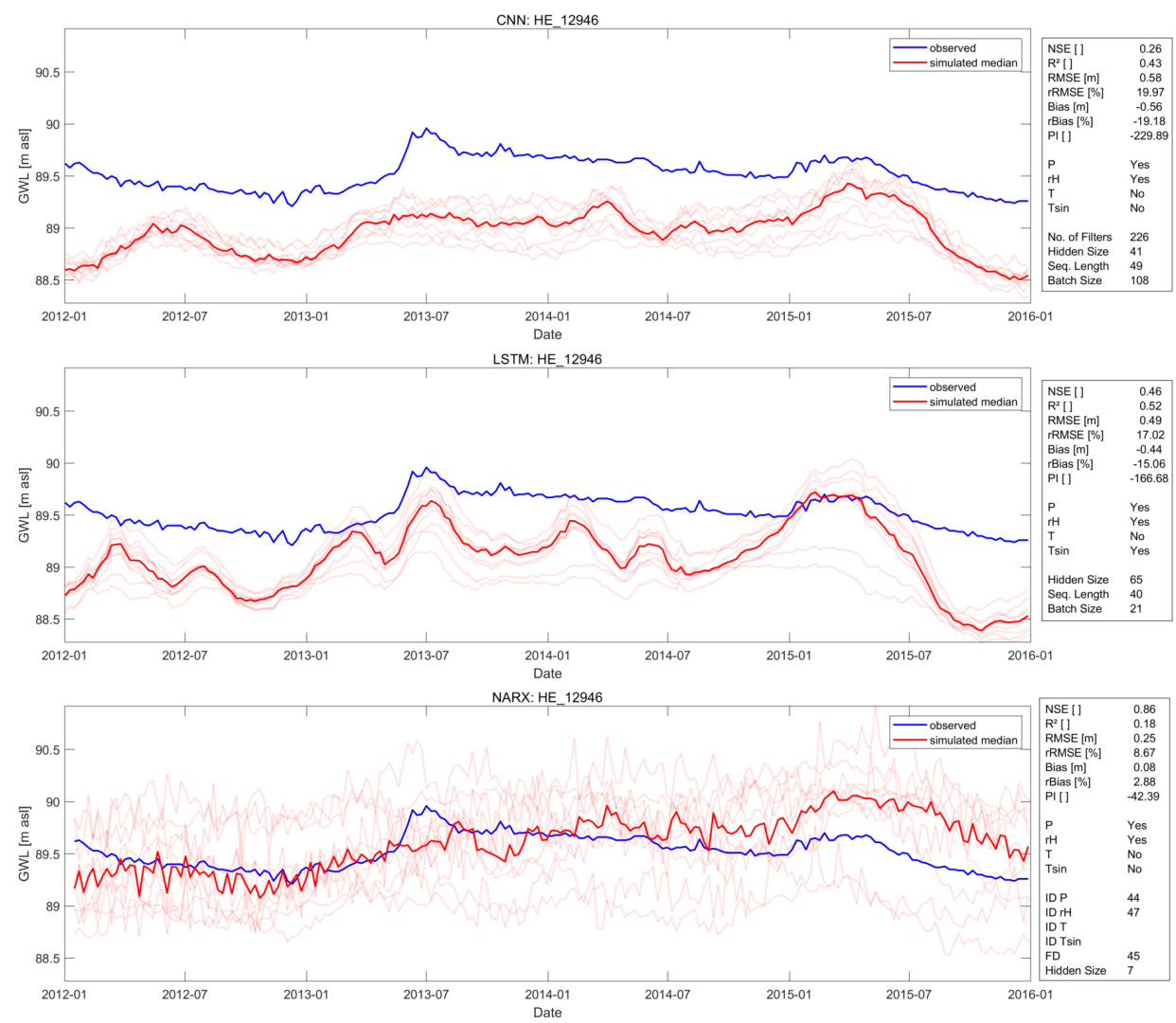

Figure S13: Seq2Val test results for well HE_12946 

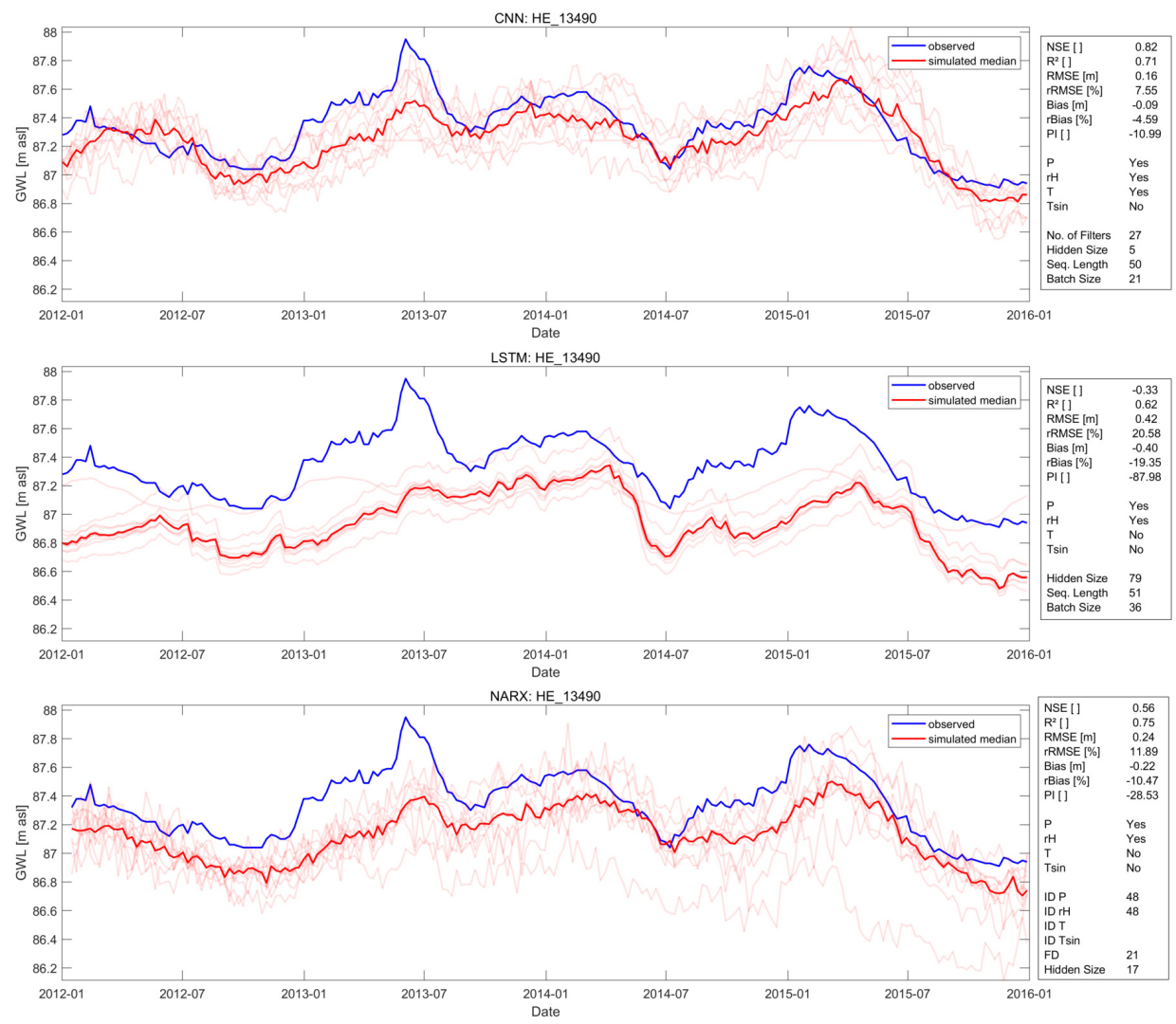

Figure S14: Seq2Val test results for well HE_13490 

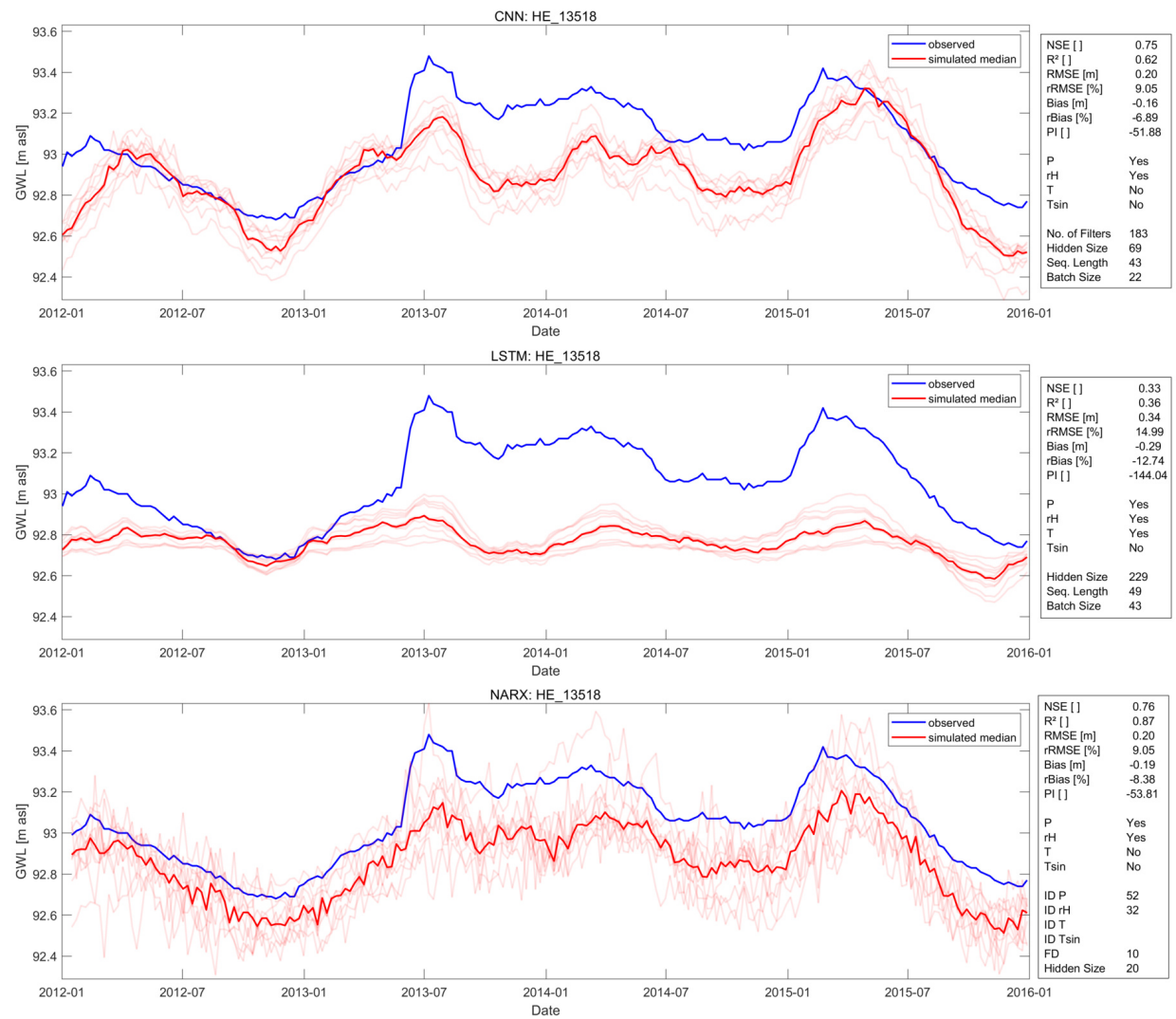

Figure S15: Seq2Val test results for well HE_13518 

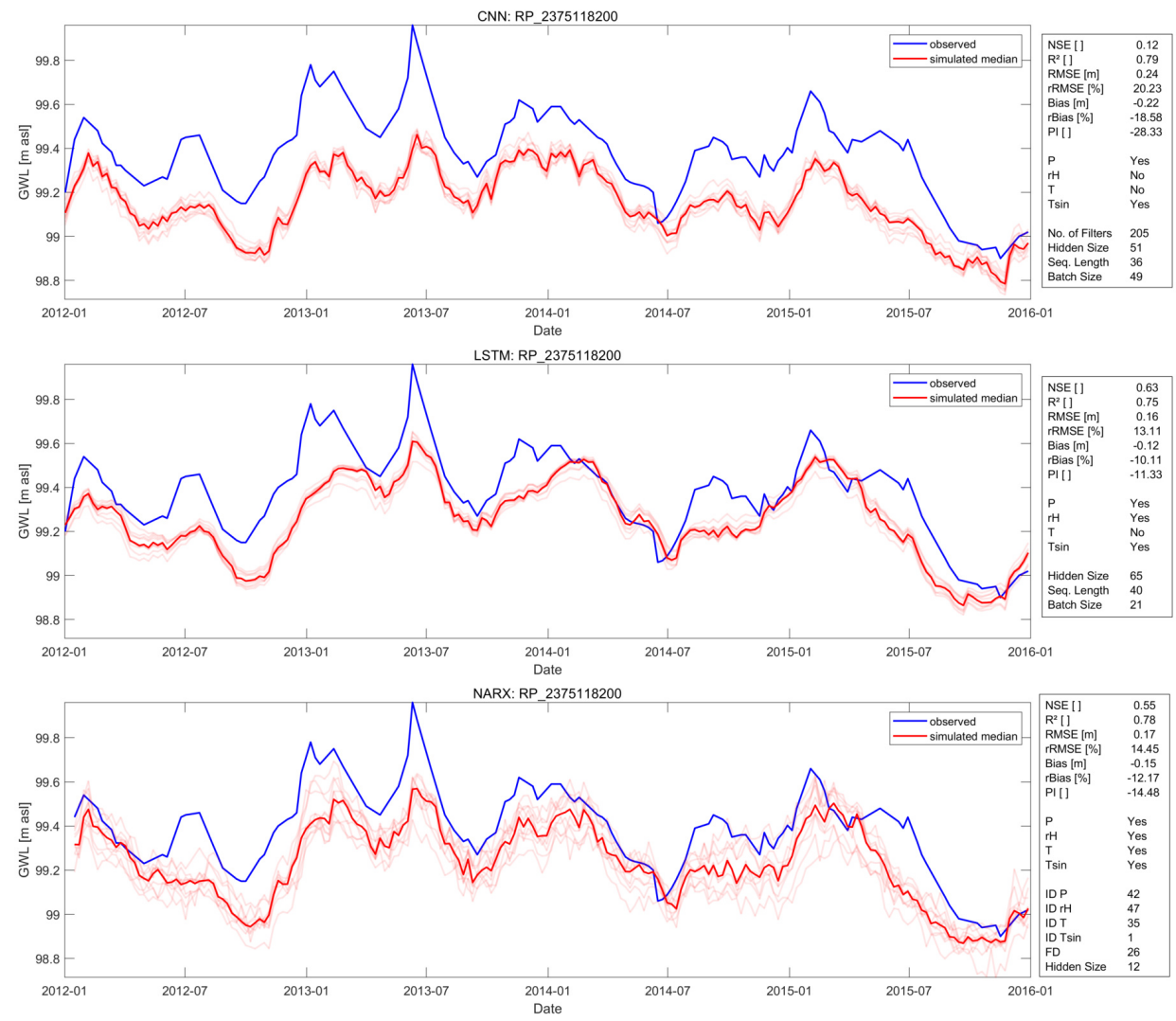

Figure S16: Seq2Val test results for well RP_2375118200 

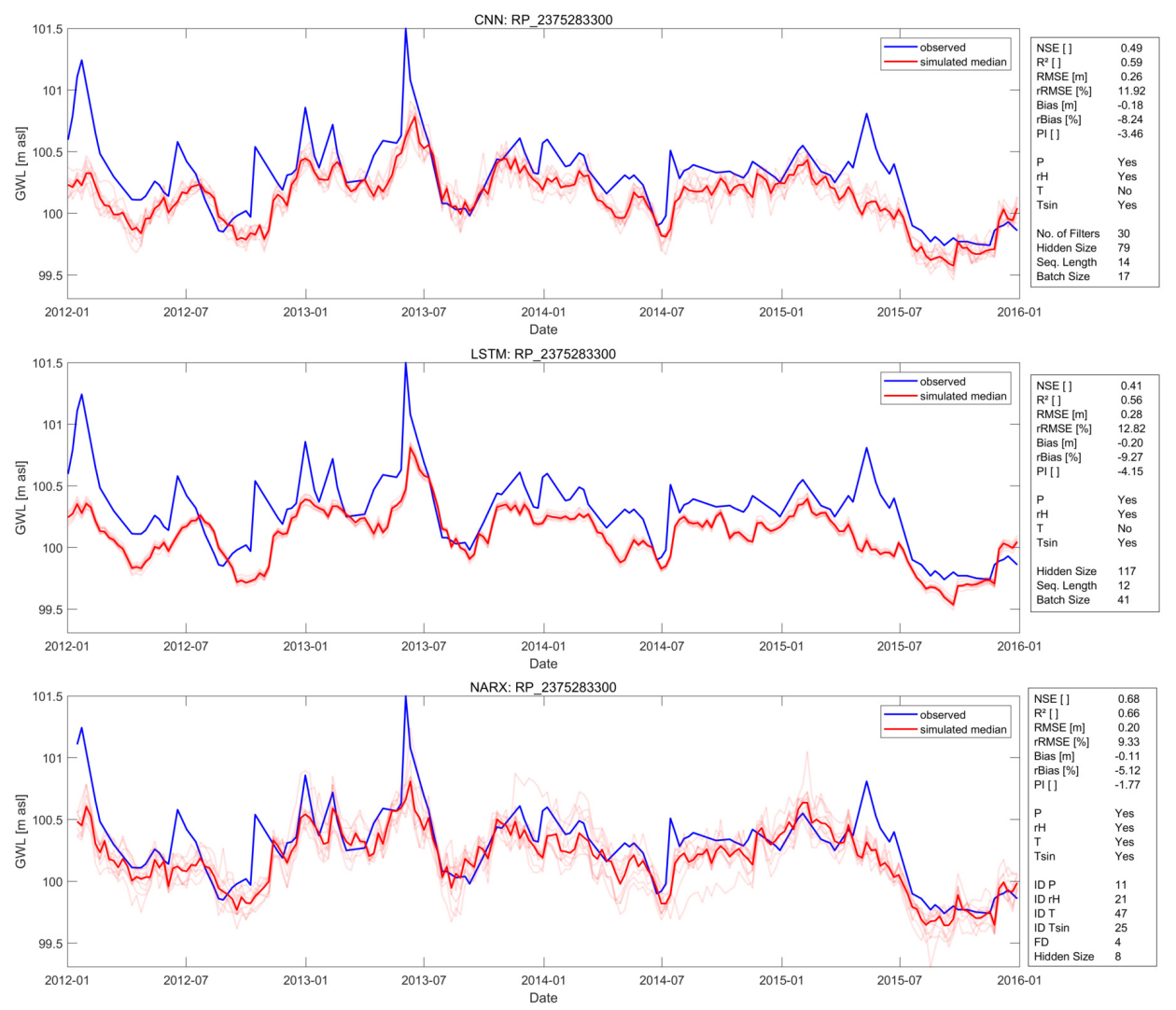

Figure S17: Seq2Val test results for well RP_2375283300 

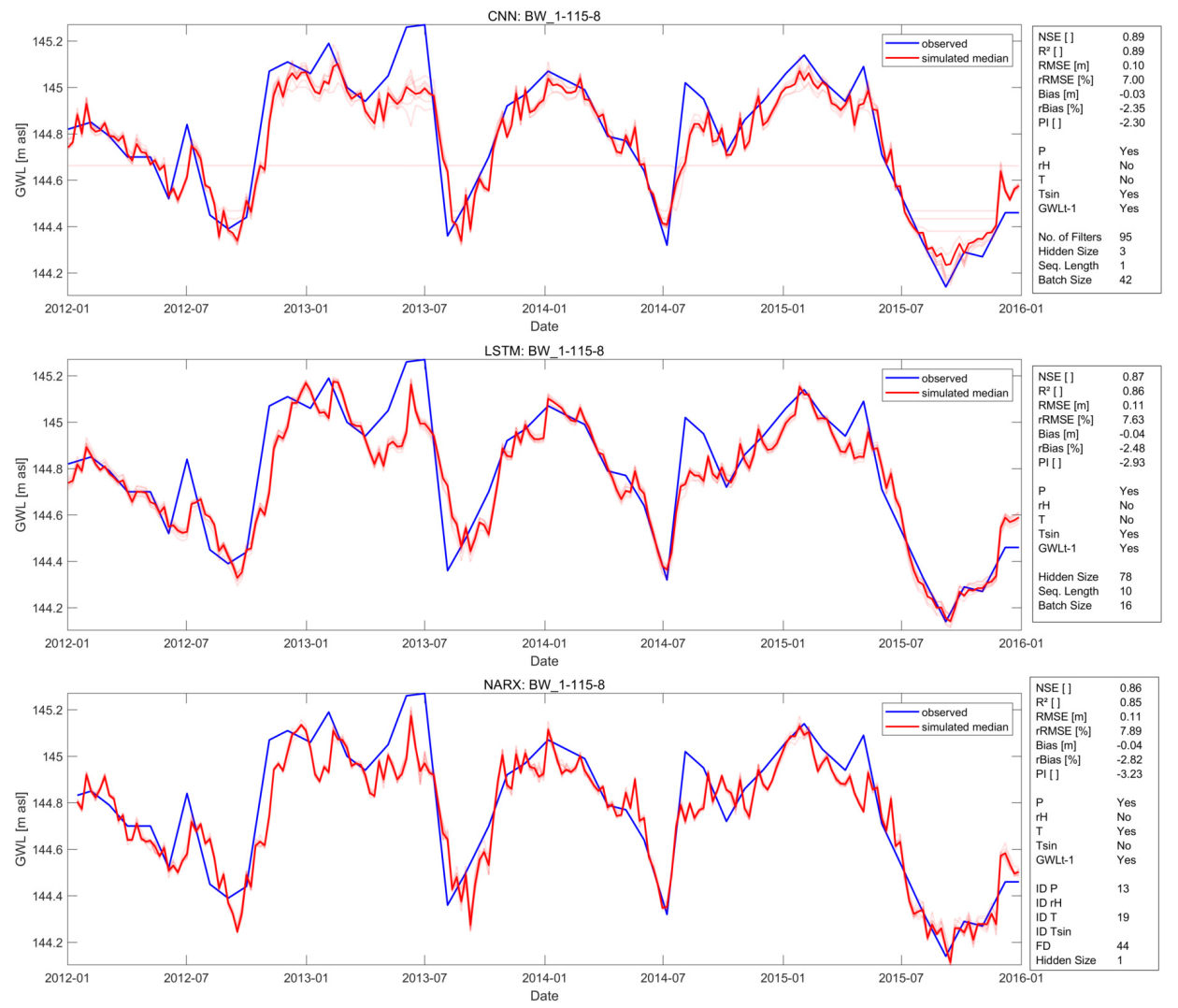

Figure S18: Seq2Val GWL $\mathrm{G}_{t-1}$ test results for well BW_1-115-8 

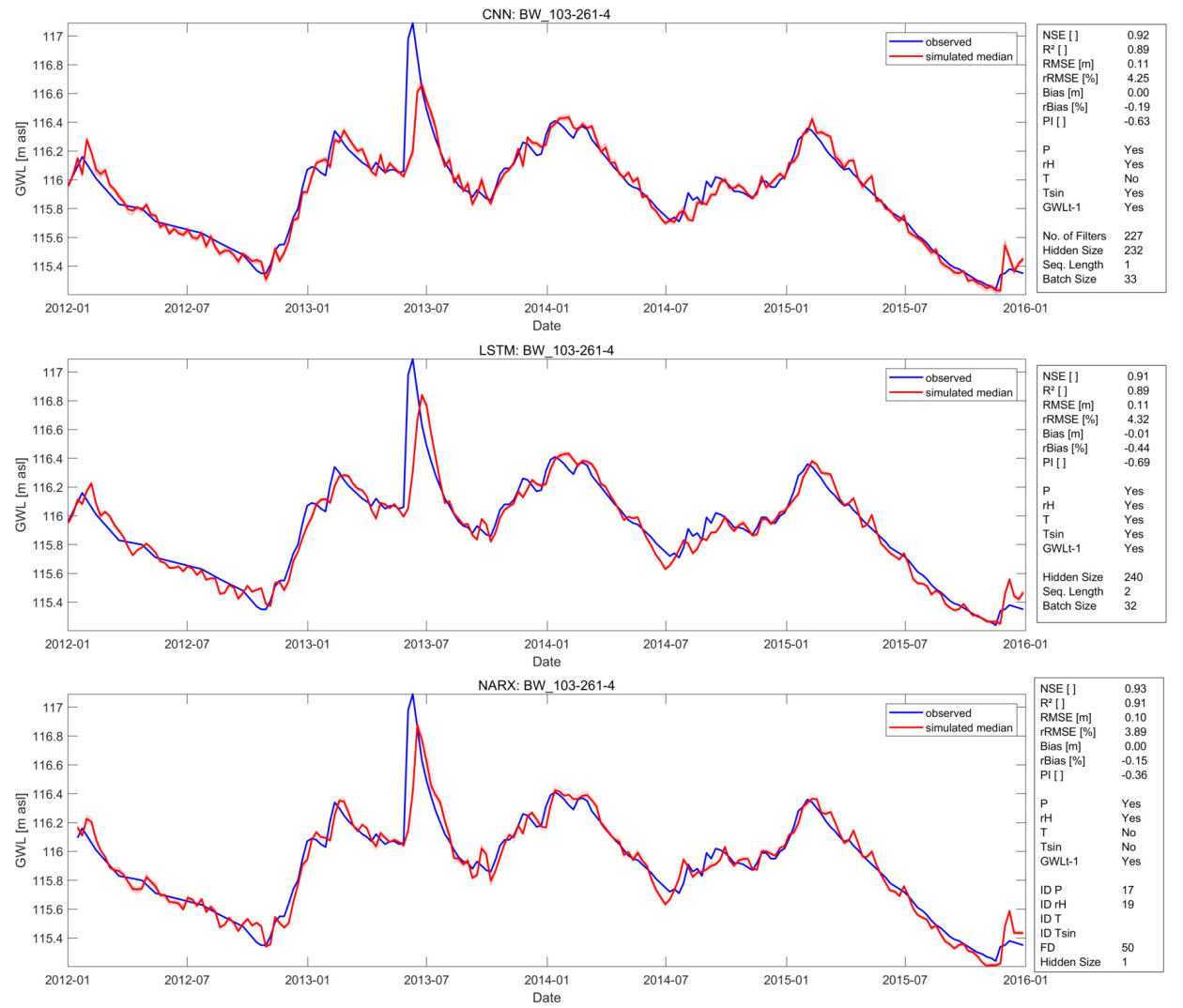

Figure S19: Seq2Val GWL $\mathrm{G}_{t-1}$ test results for well BW_103-261-4 

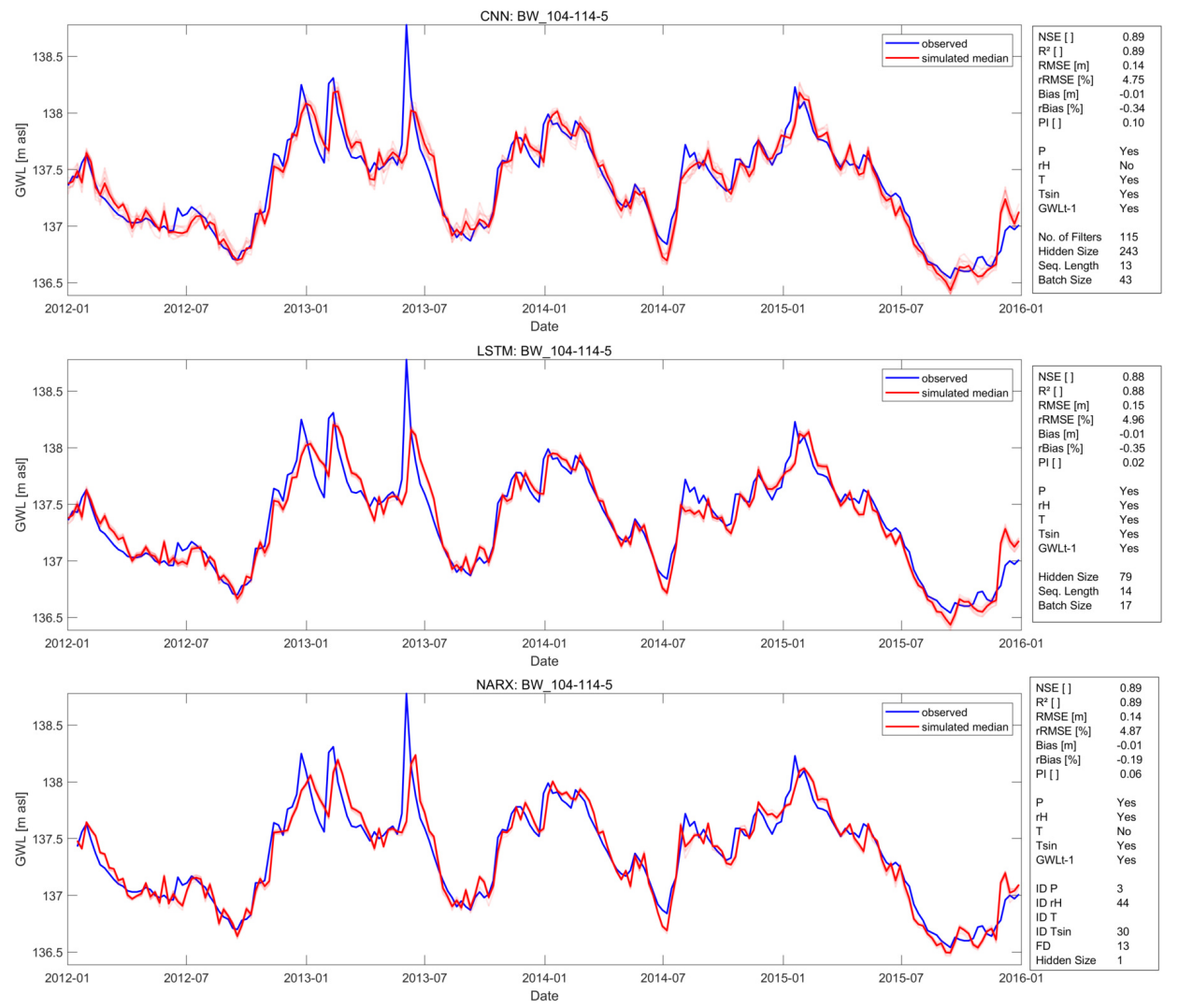

Figure S20: Seq2Val GWL $\mathrm{L}_{t-1}$ test results for well BW_104-114-5 

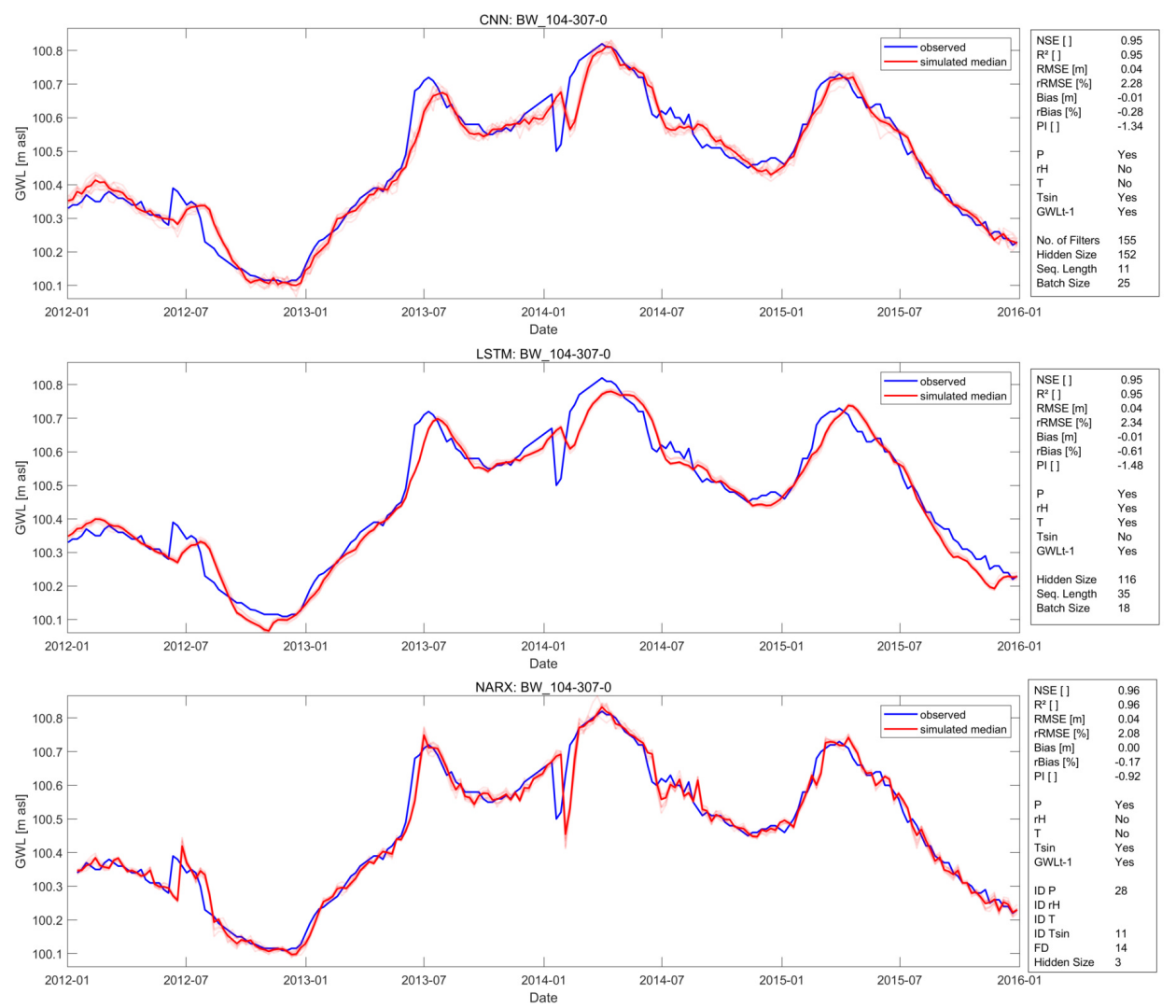

Figure S21: Seq2Val GWL $\mathrm{G}_{t-1}$ test results for well BW_104-307-0 

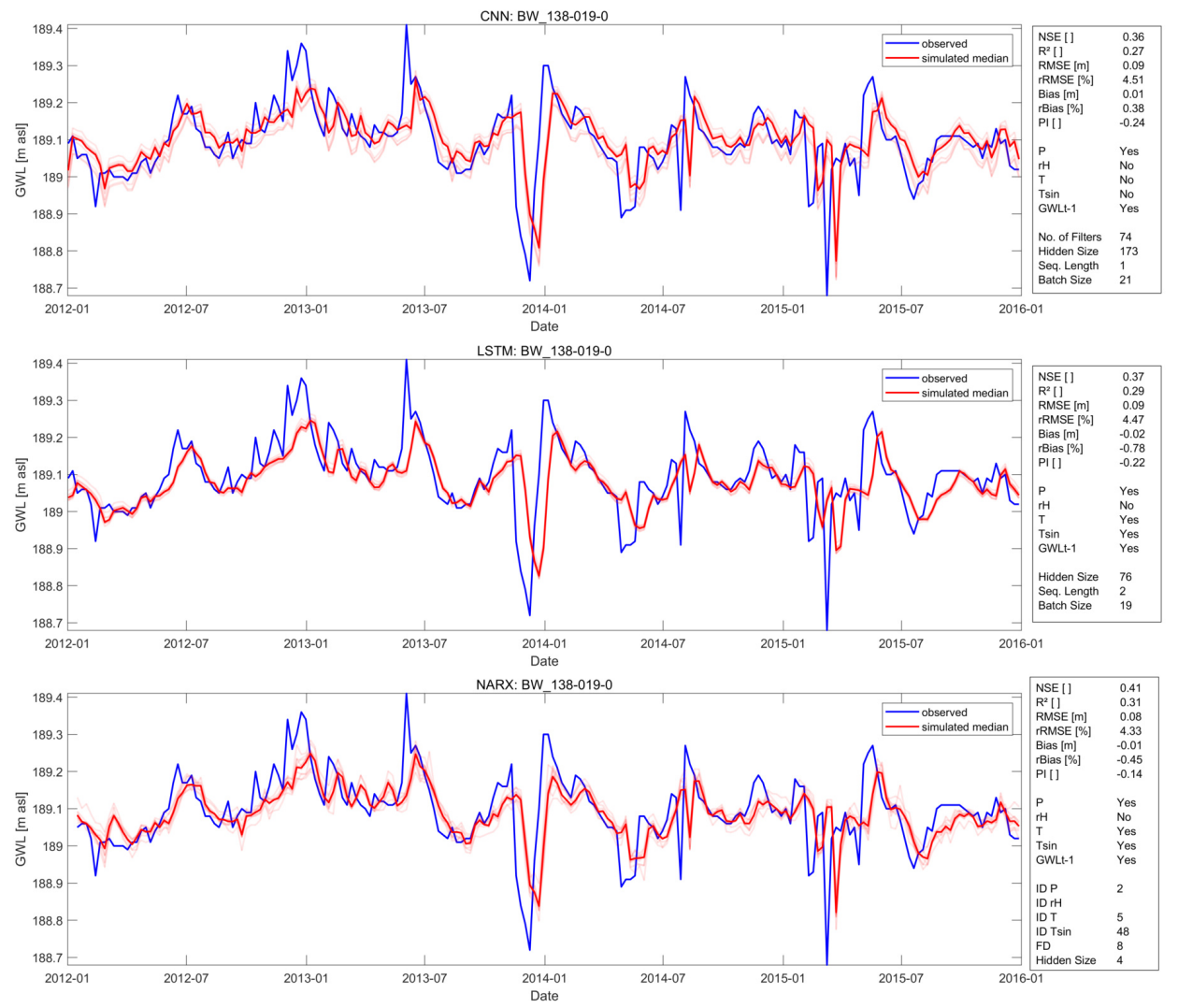

Figure S22: Seq2Val GWL $\mathrm{L}_{t-1}$ test results for well BW_138-019-0 

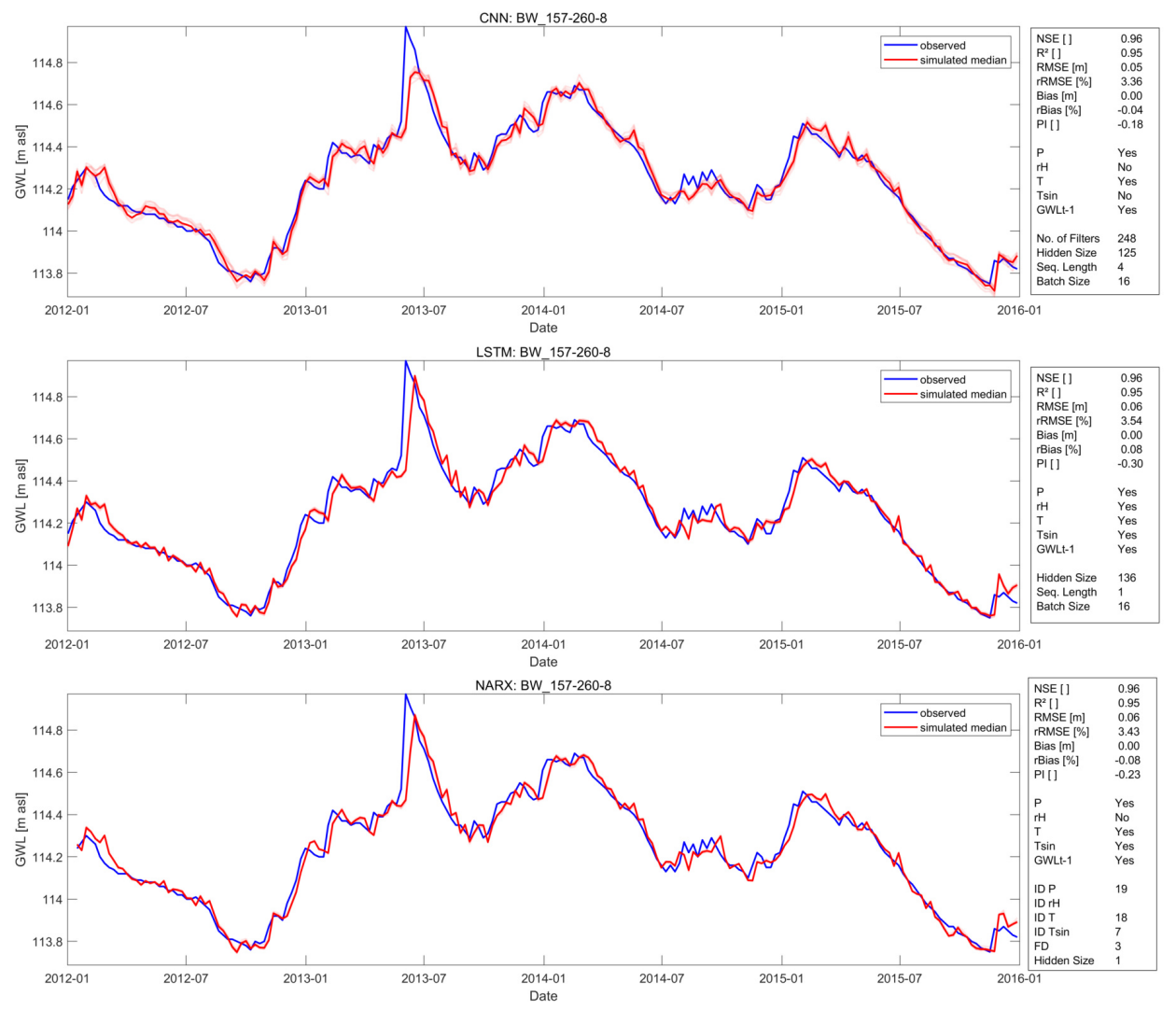

Figure S23: Seq2Val GWL $\mathrm{G}_{t-1}$ test results for well BW_157-260-8 

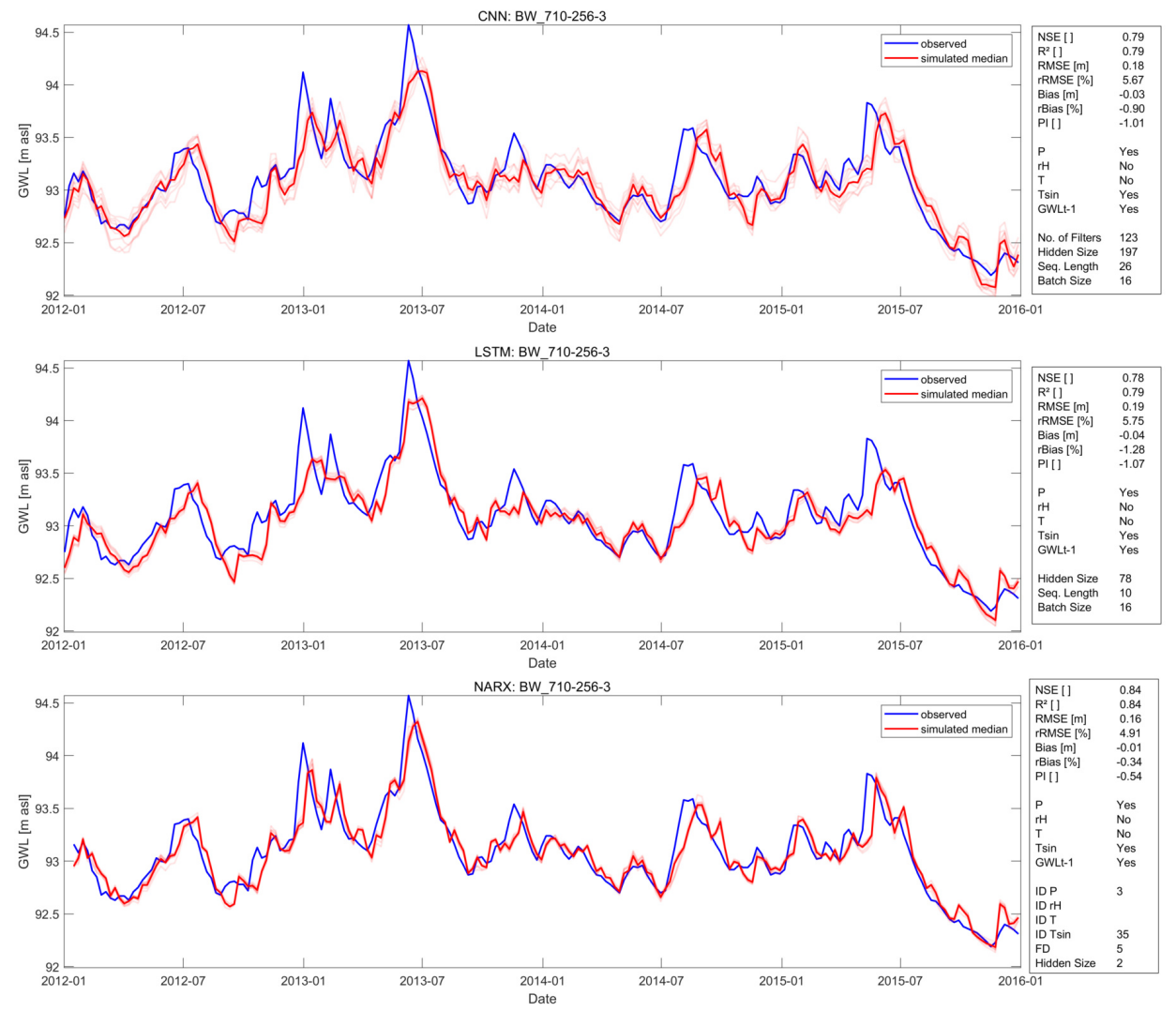

Figure S24: Seq2Val GWL $\mathrm{L}_{t-1}$ test results for well BW_710-256-3 

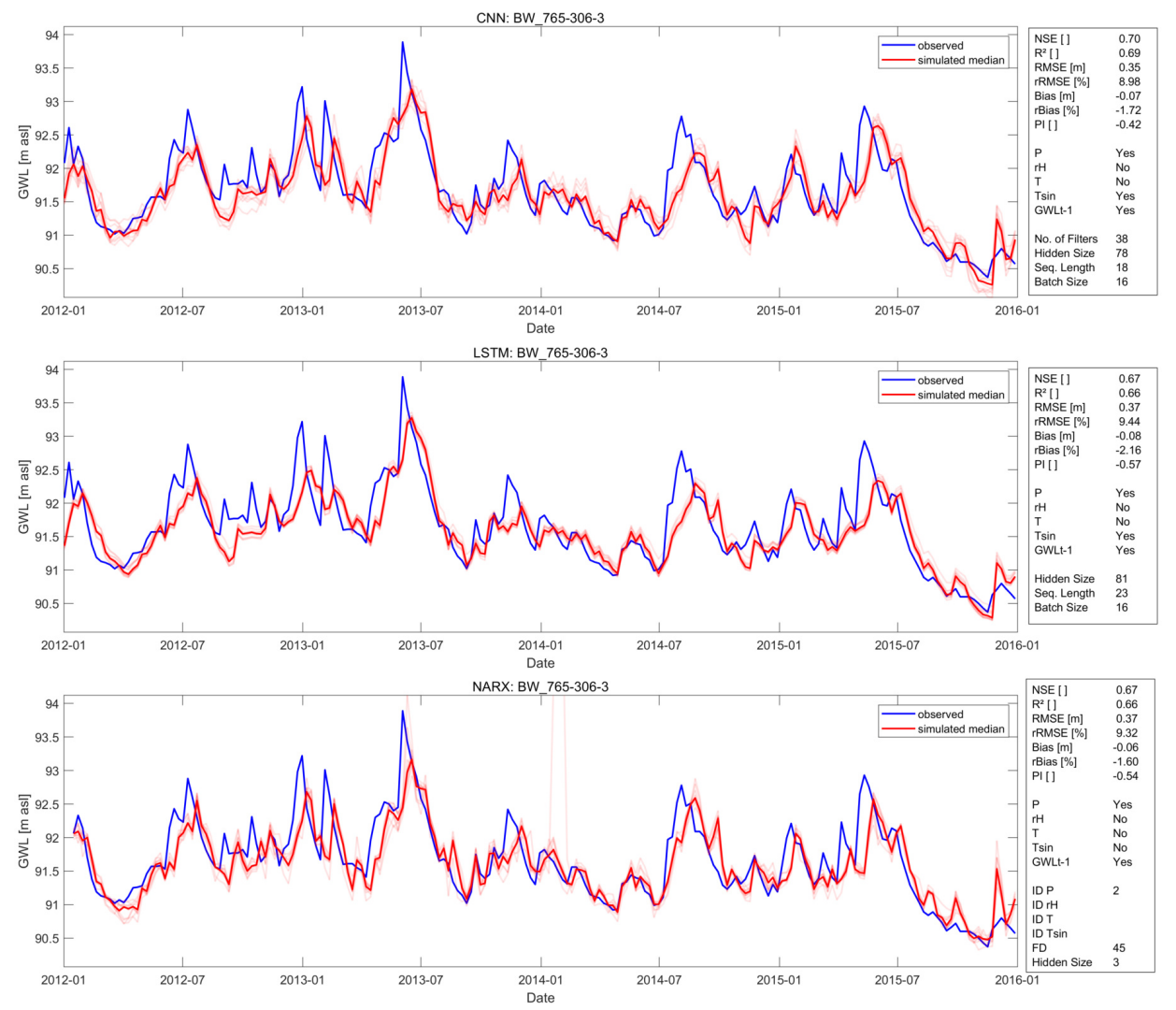

Figure S25: Seq2Val GWL $\mathrm{L}_{t-1}$ test results for well BW_765-306-3 

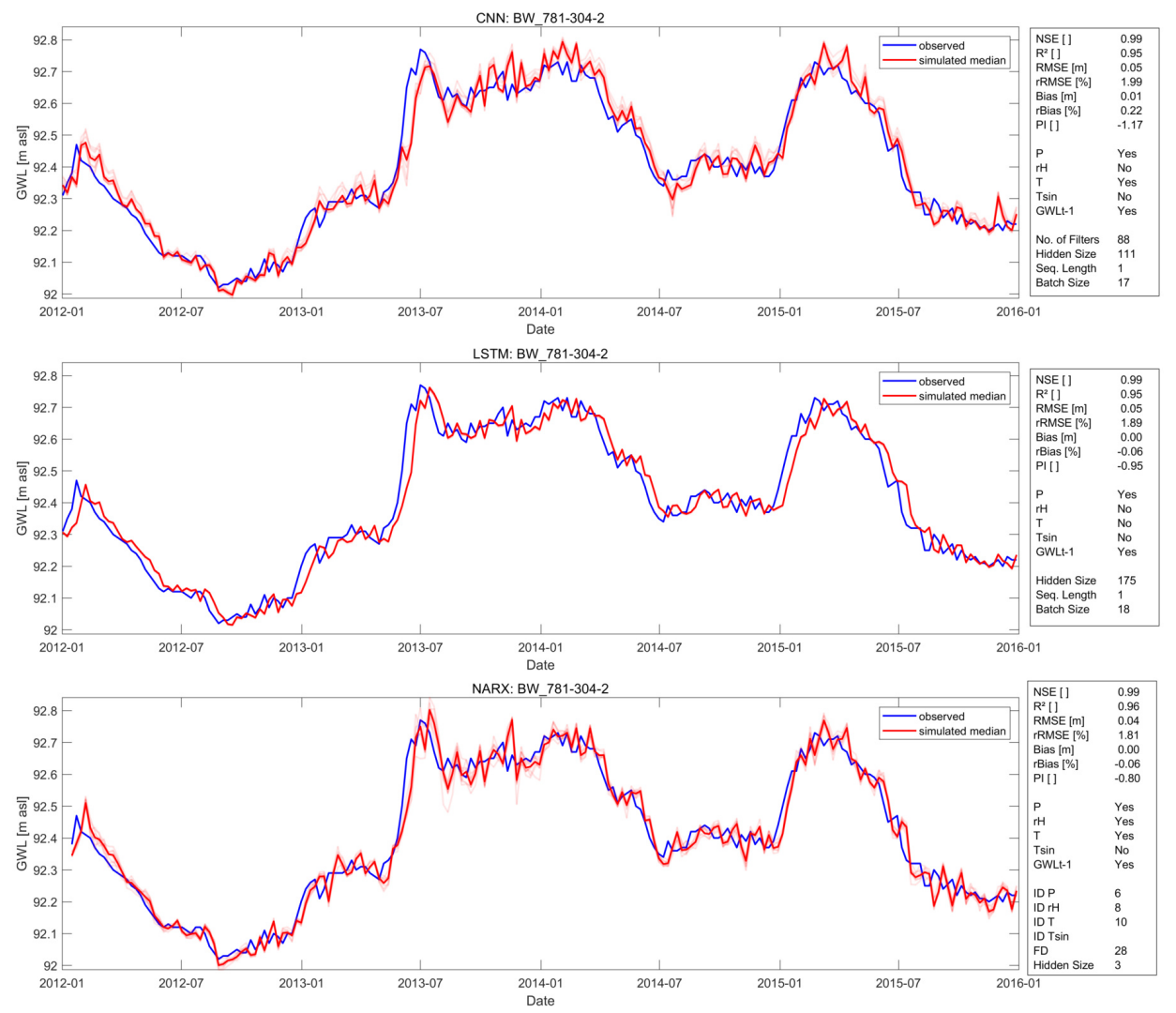

Figure S26: Seq2Val GWL $\mathrm{GW}_{t-1}$ test results for well BW_781-304-2 

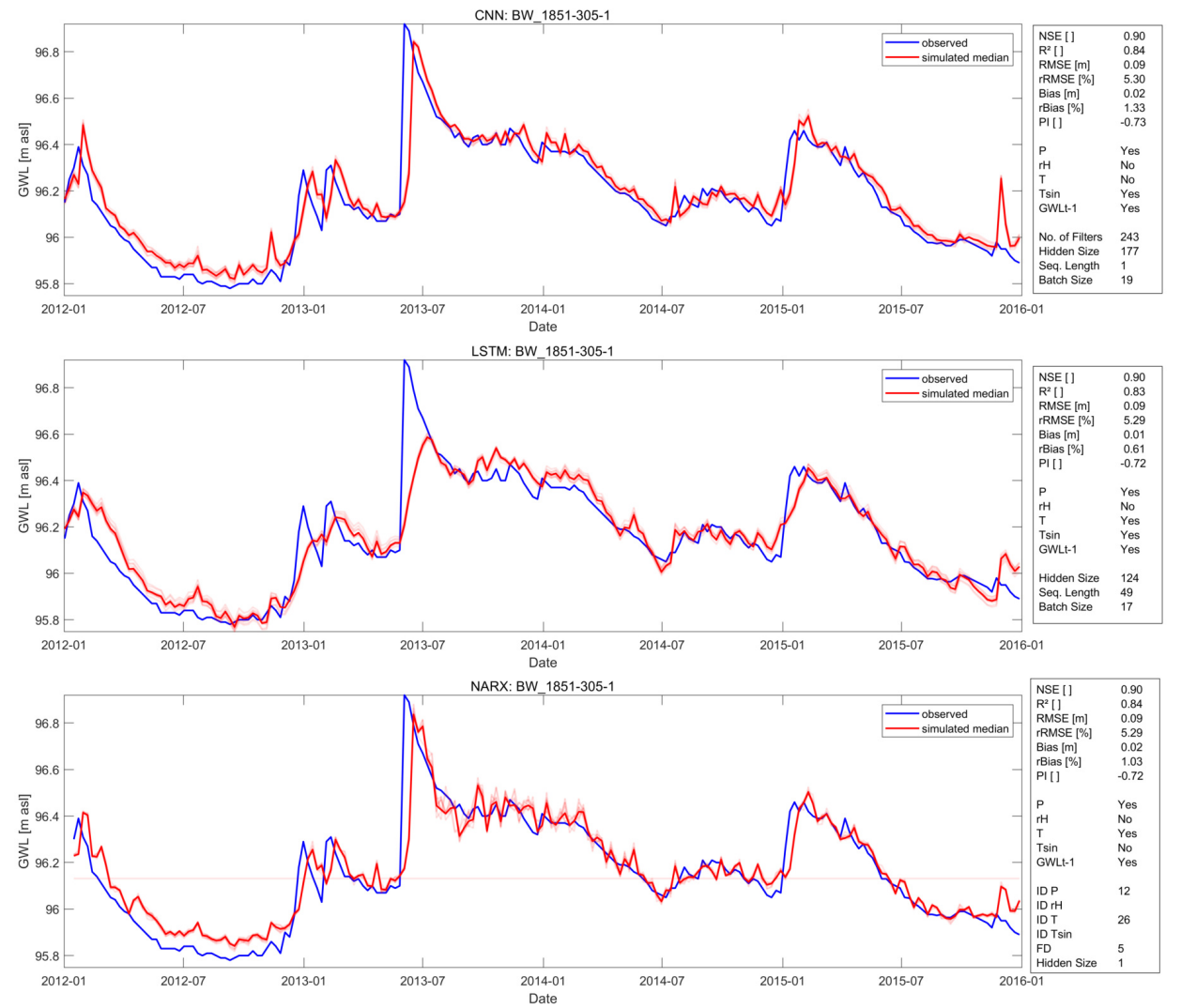

Figure S27: Seq2Val GWL $\mathrm{L}_{t-1}$ test results for well BW_1851-305-1 

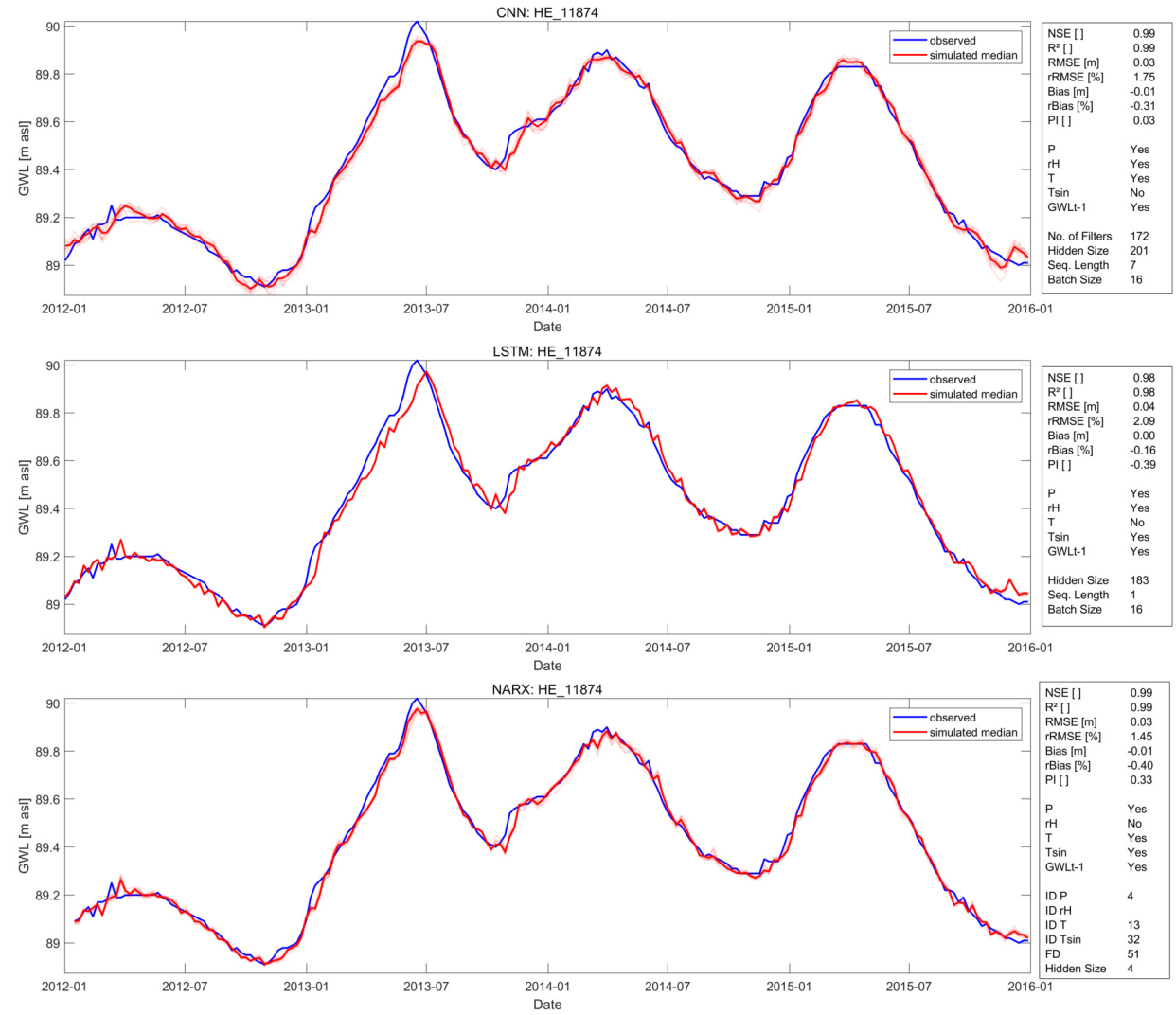

Figure S28: Seq2Val GWL $\mathrm{GW}_{t-1}$ test results for well HE_11874 

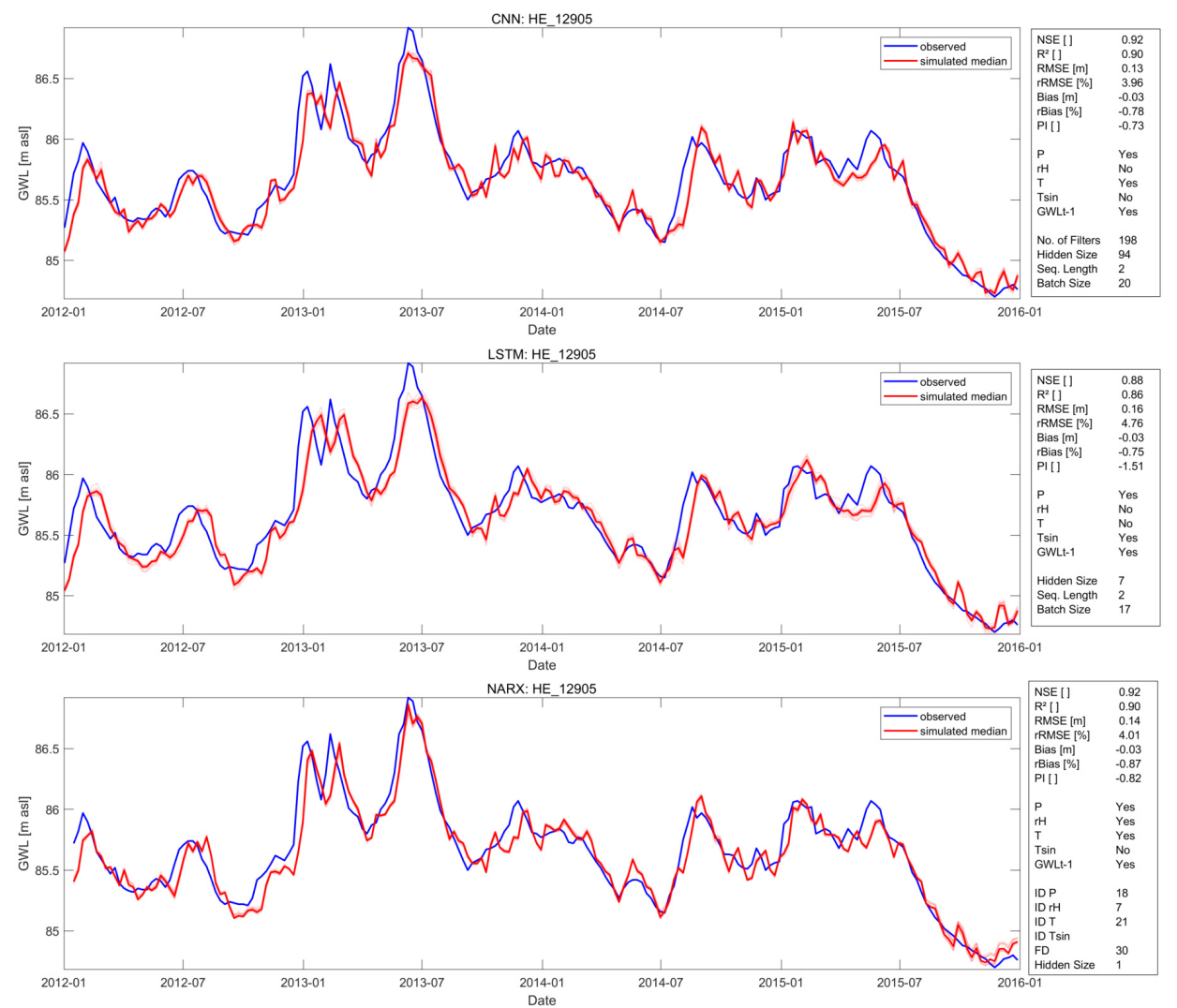

Figure S29: Seq2Val GWL $\mathrm{GW}_{t-1}$ test results for well HE_12905 

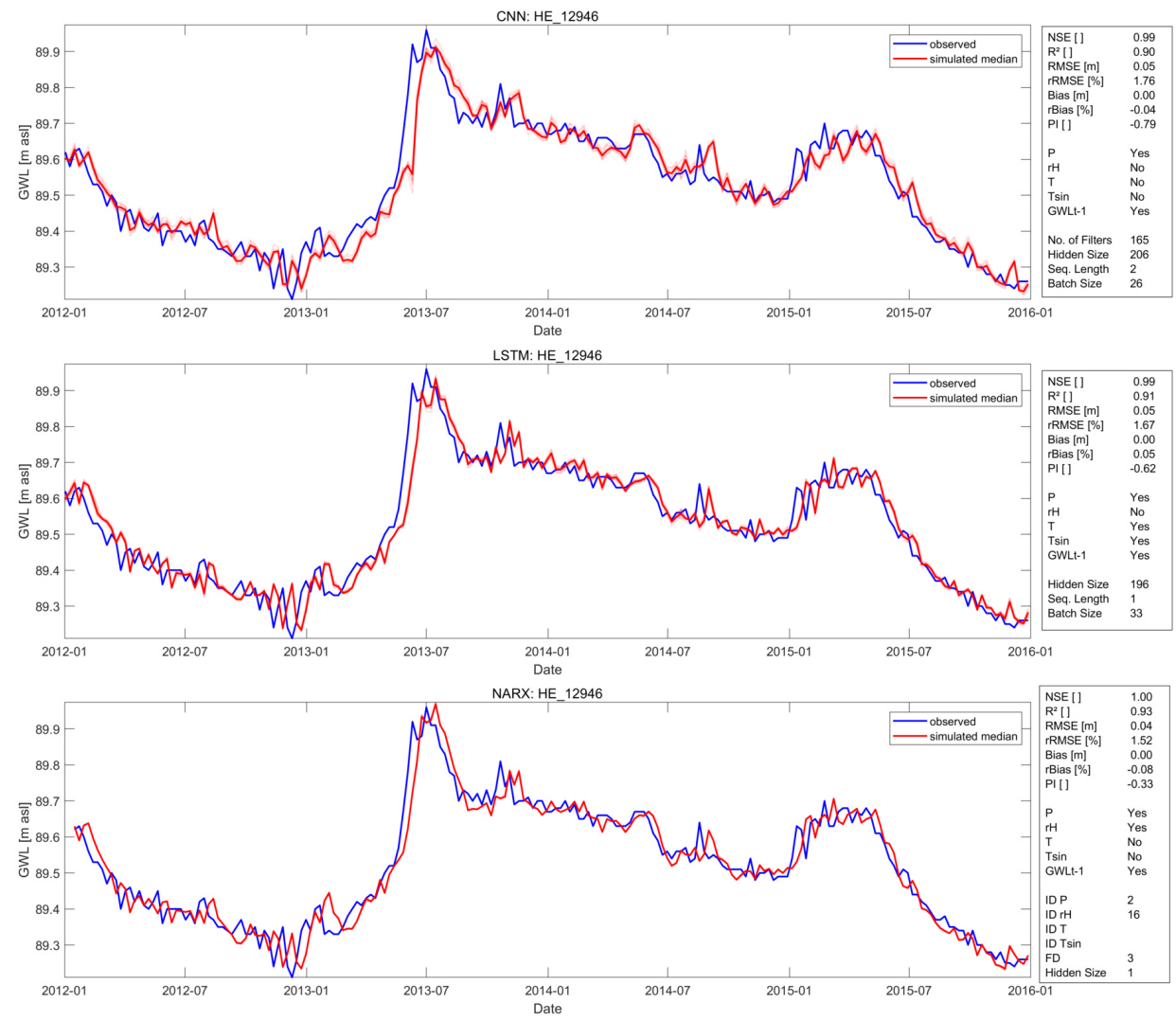

Figure S30: Seq2Val GWL $\mathrm{GW}_{t-1}$ test results for well HE_12946 

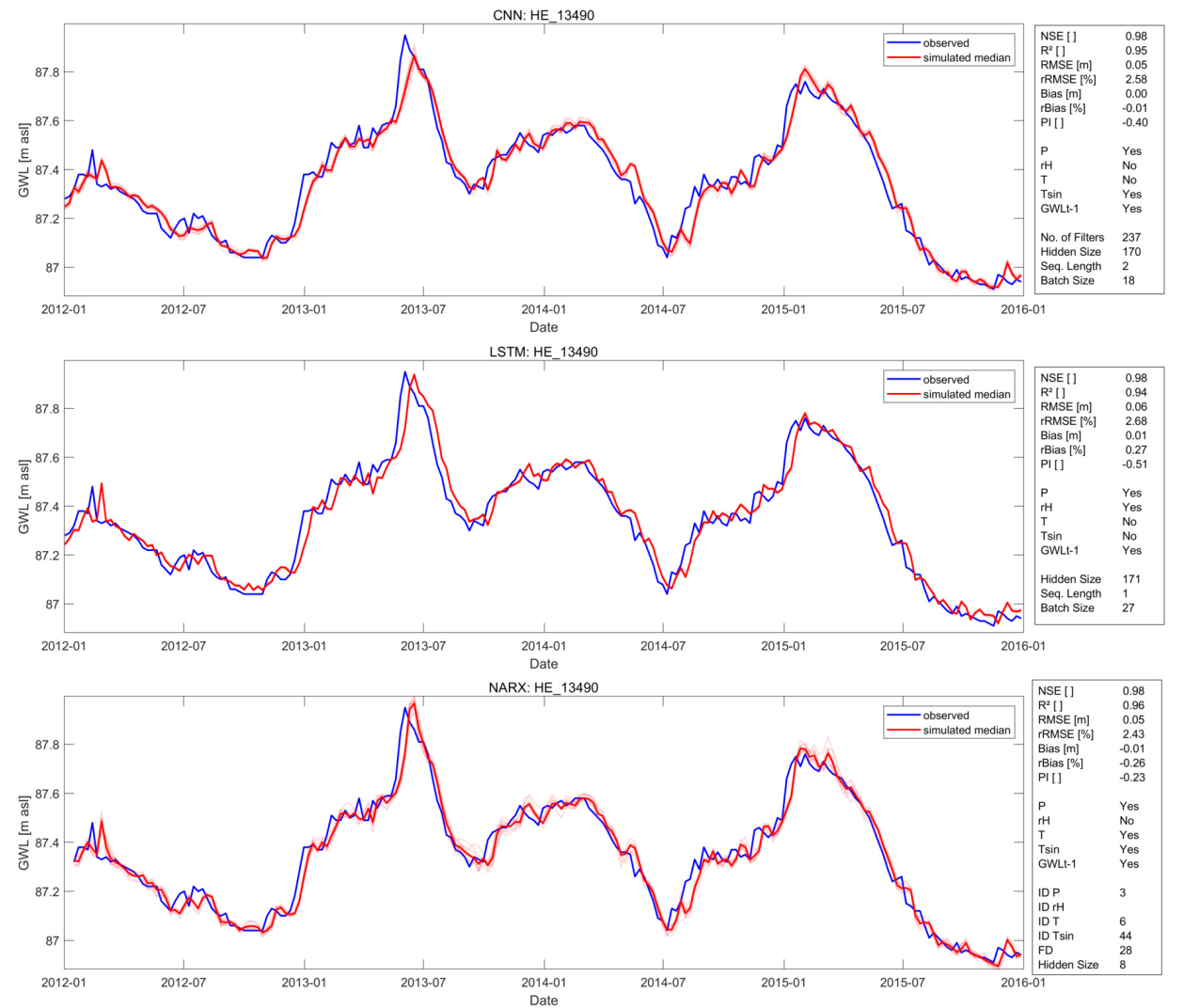

Figure S31: Seq2Val GWL $\mathrm{GW}_{t-1}$ test results for well HE_13490 

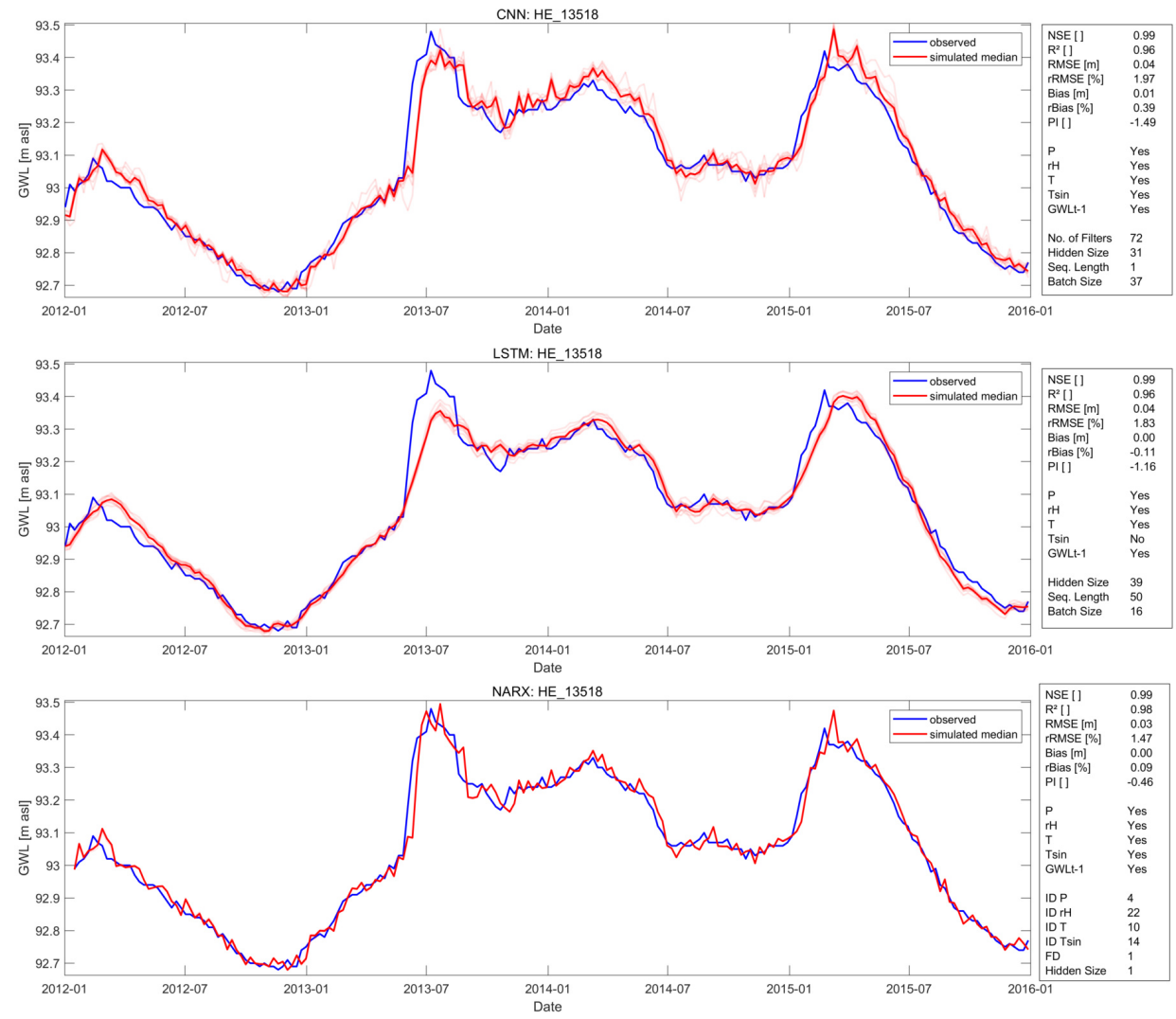

Figure S32: Seq2Val GWL $\mathrm{GW}_{t-1}$ test results for well HE_13518 

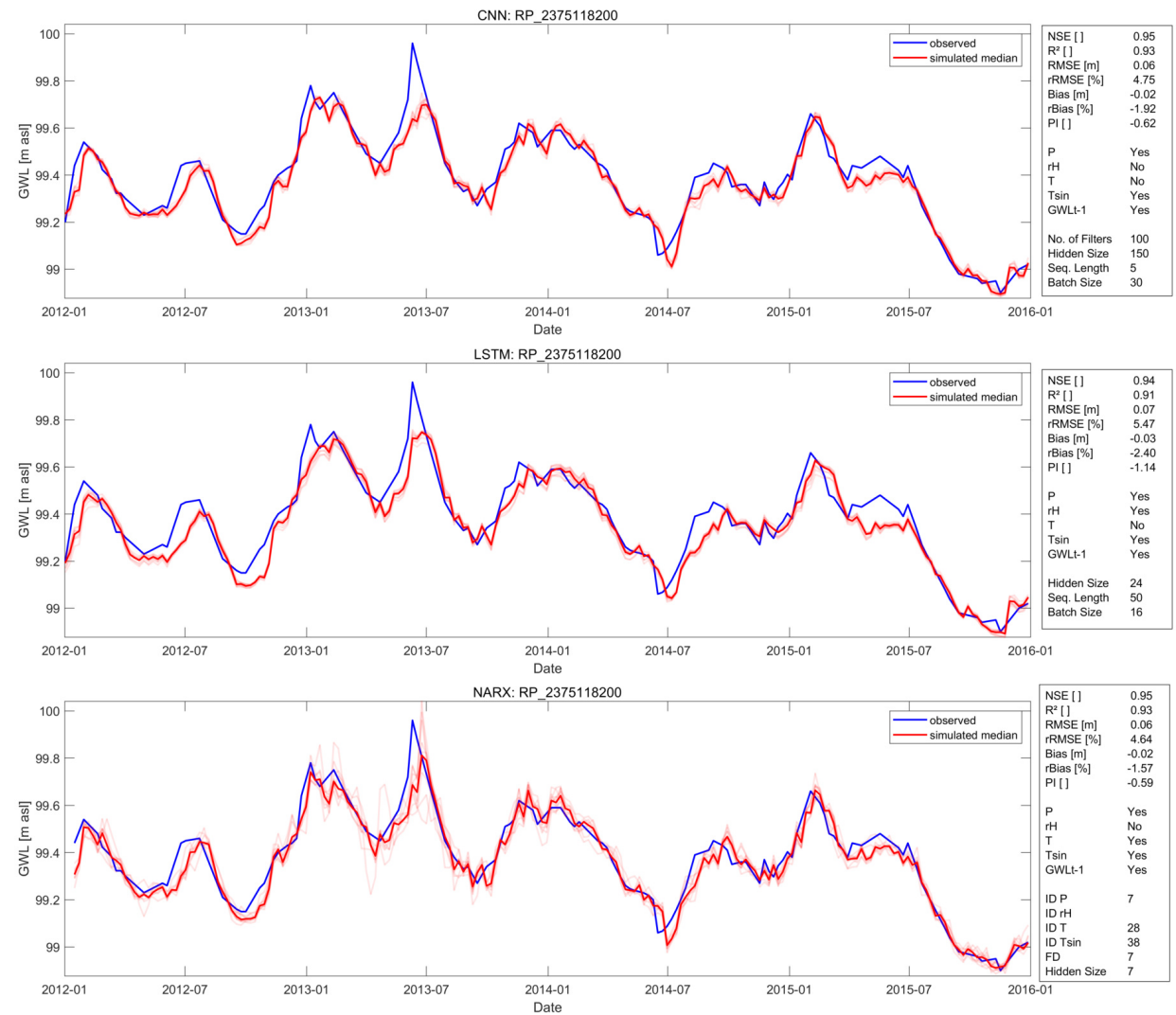

Figure S33: Seq2Val GWL $\mathrm{G}_{t-1}$ test results for well RP_2375118200 

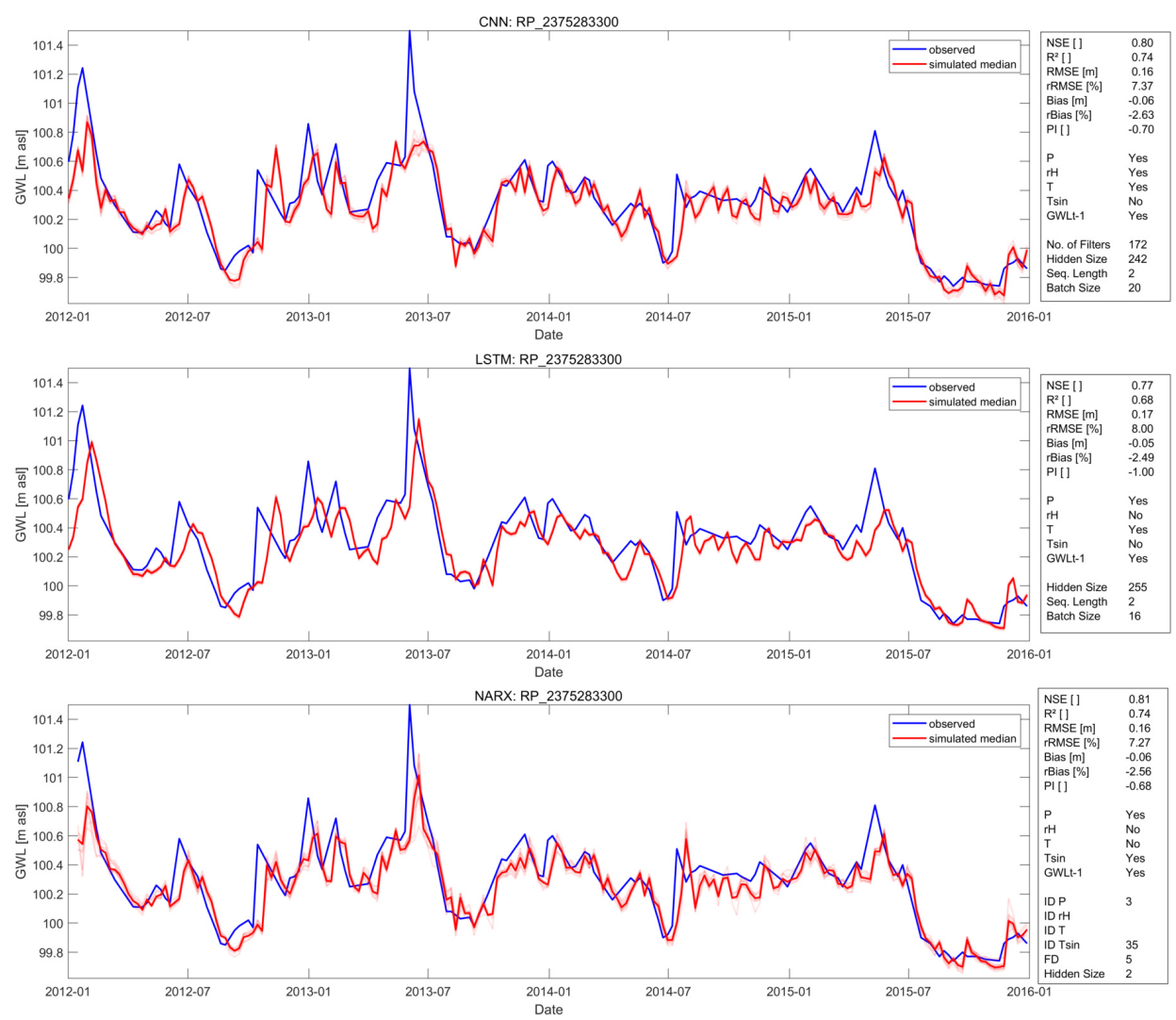

Figure S34: Seq2Val GWL $\mathrm{G}_{t-1}$ test results for well RP_2375283300 

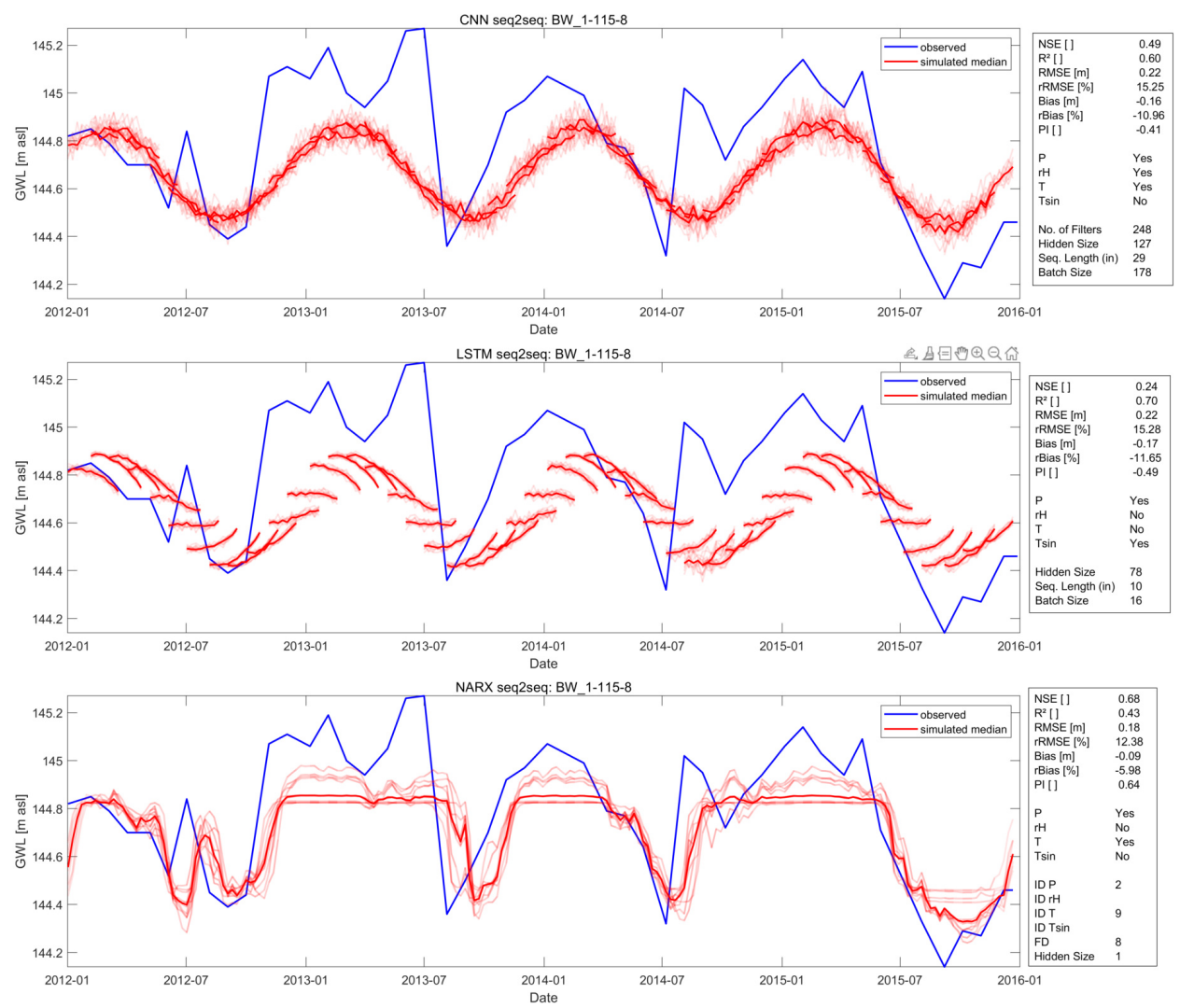

Figure S35: Seq2Seq test results for well BW_1-115-8 

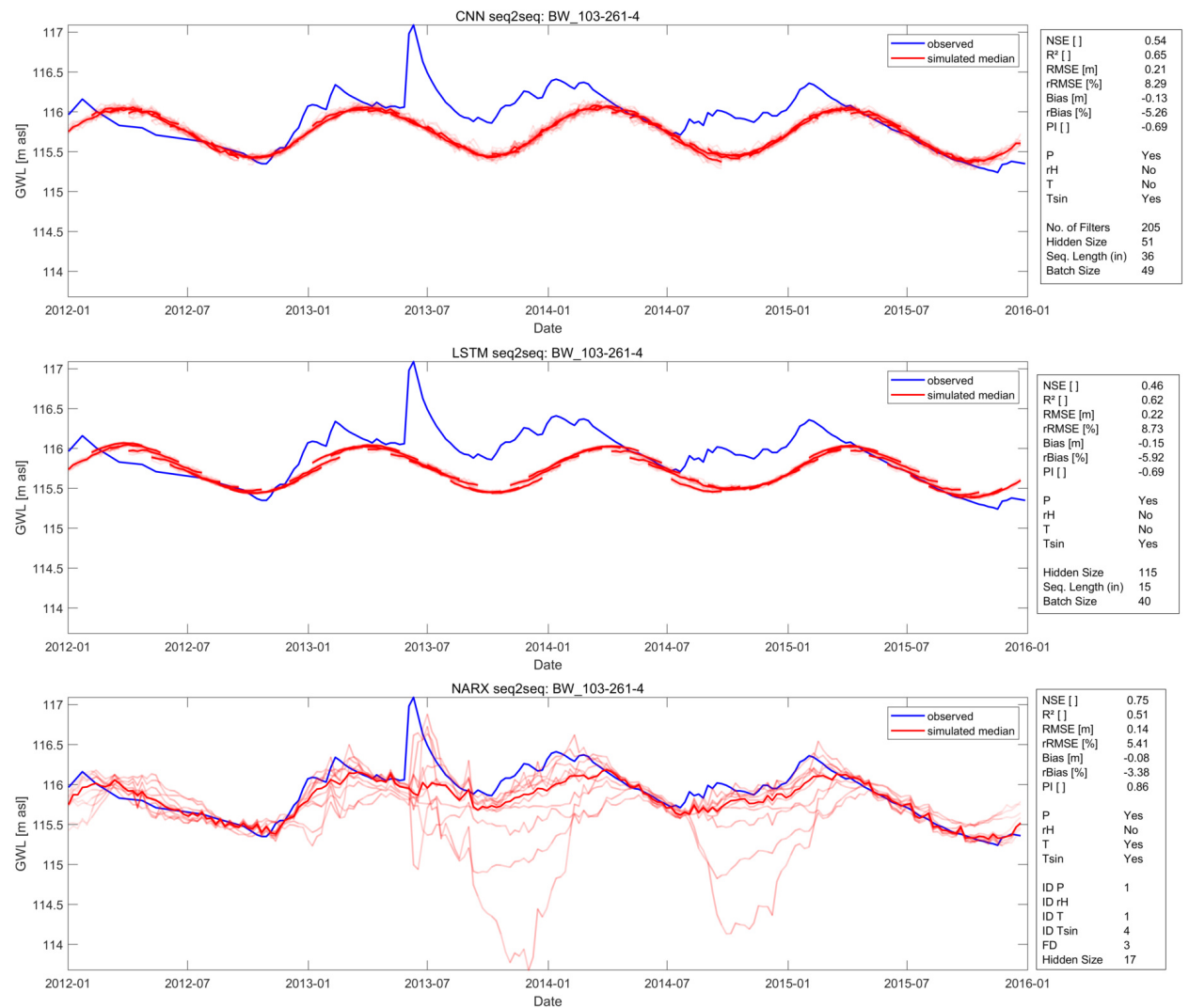

Figure S36: Seq2Seq test results for well BW_103-261-4 

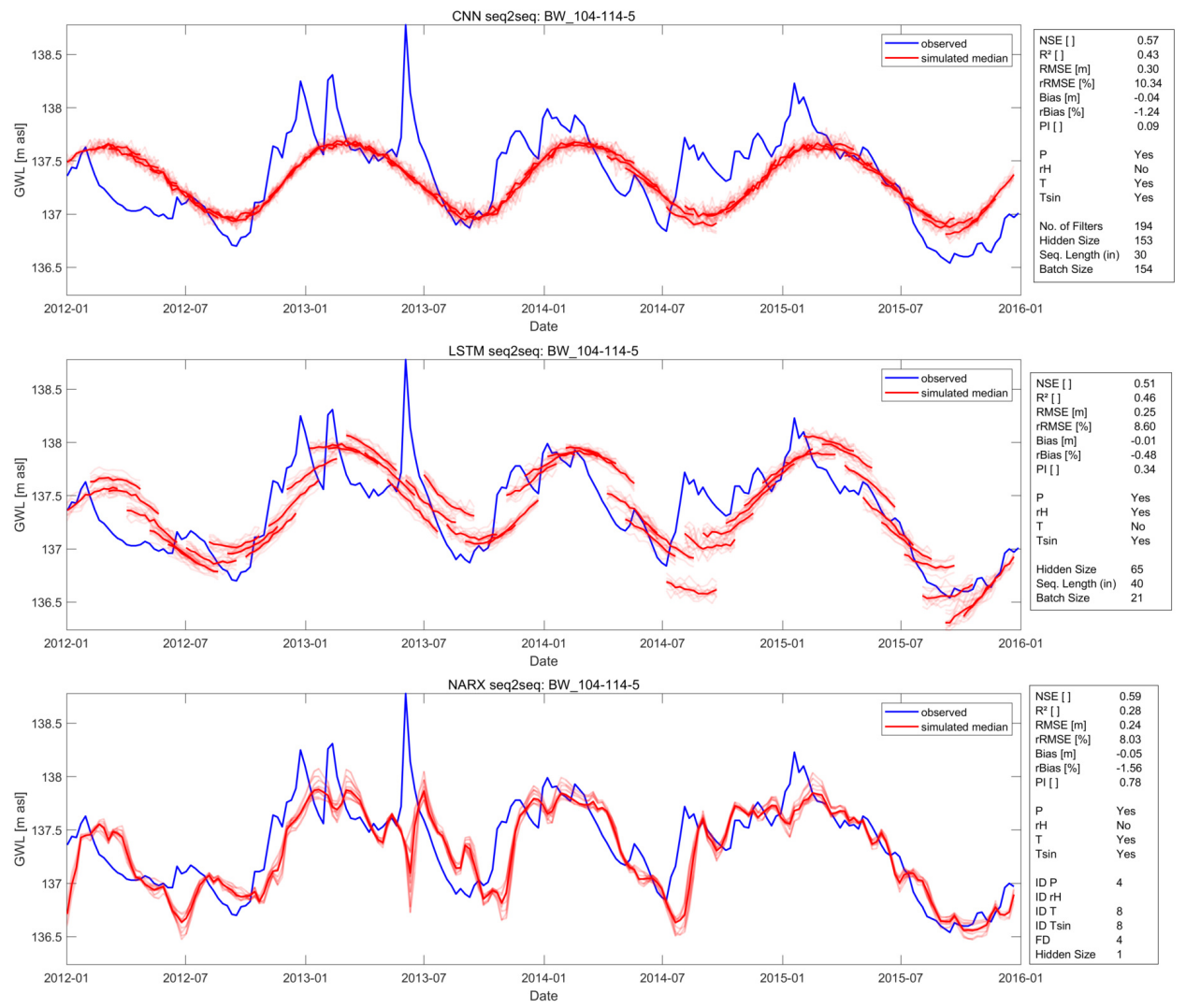

Figure S37: Seq2Seq test results for well BW_104-114-5 

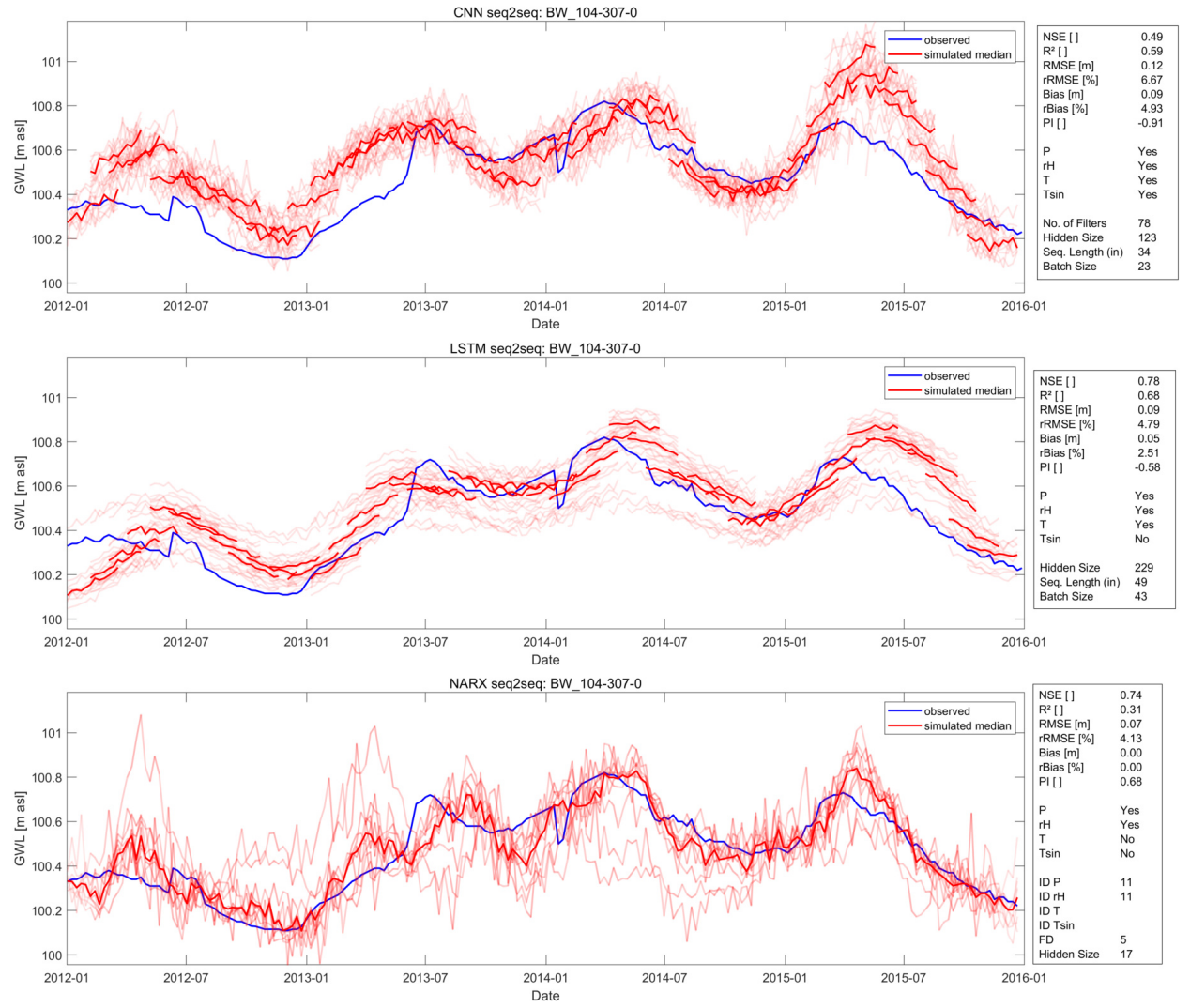

Figure S38: Seq2Seq test results for well BW_104-307-0 

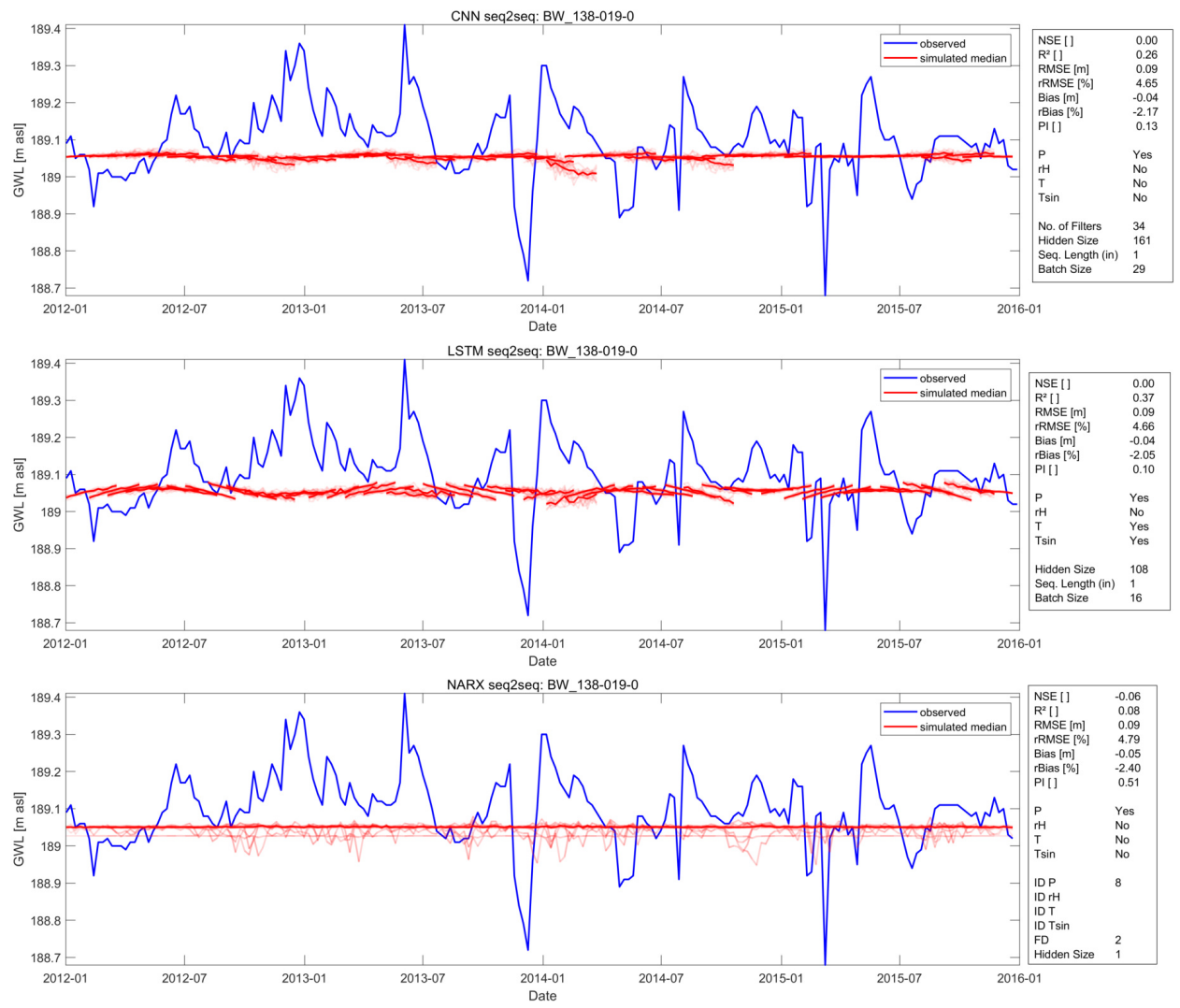

Figure S39: Seq2Seq test results for well BW_138-019-0 

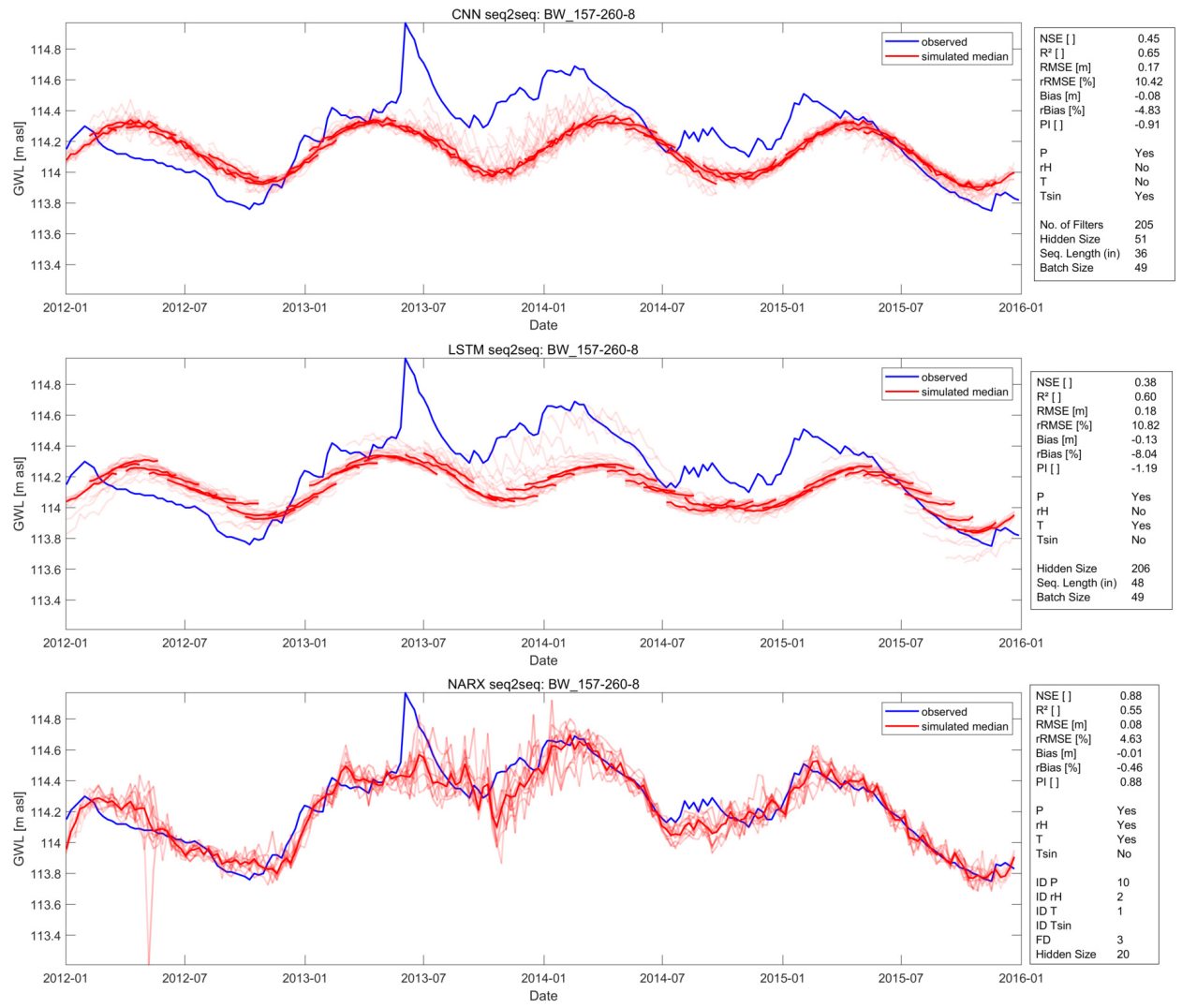

Figure S40: Seq2Seq test results for well BW_157-260-8 

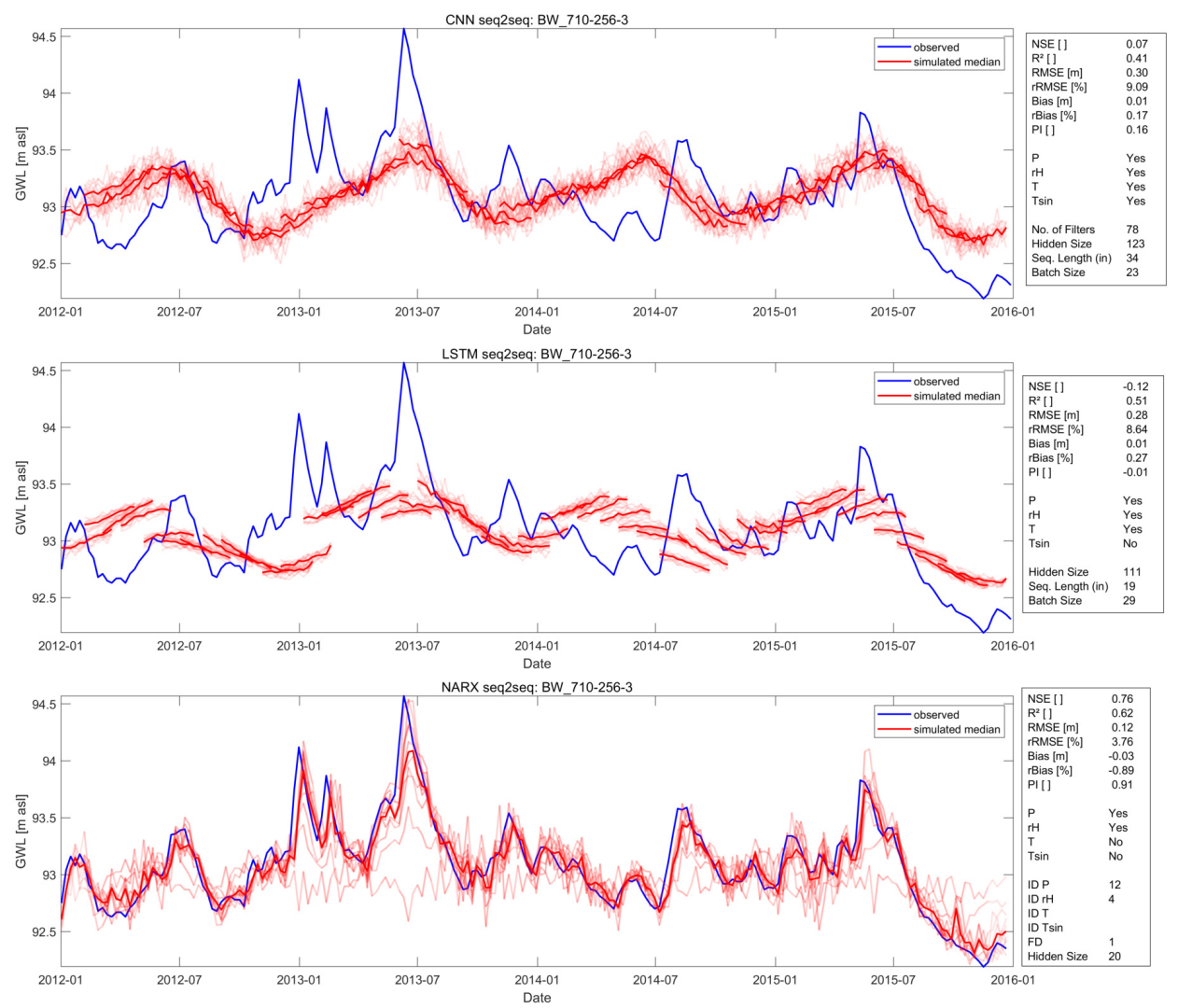

Figure S41: Seq2Seq test results for well BW_710-256-3 

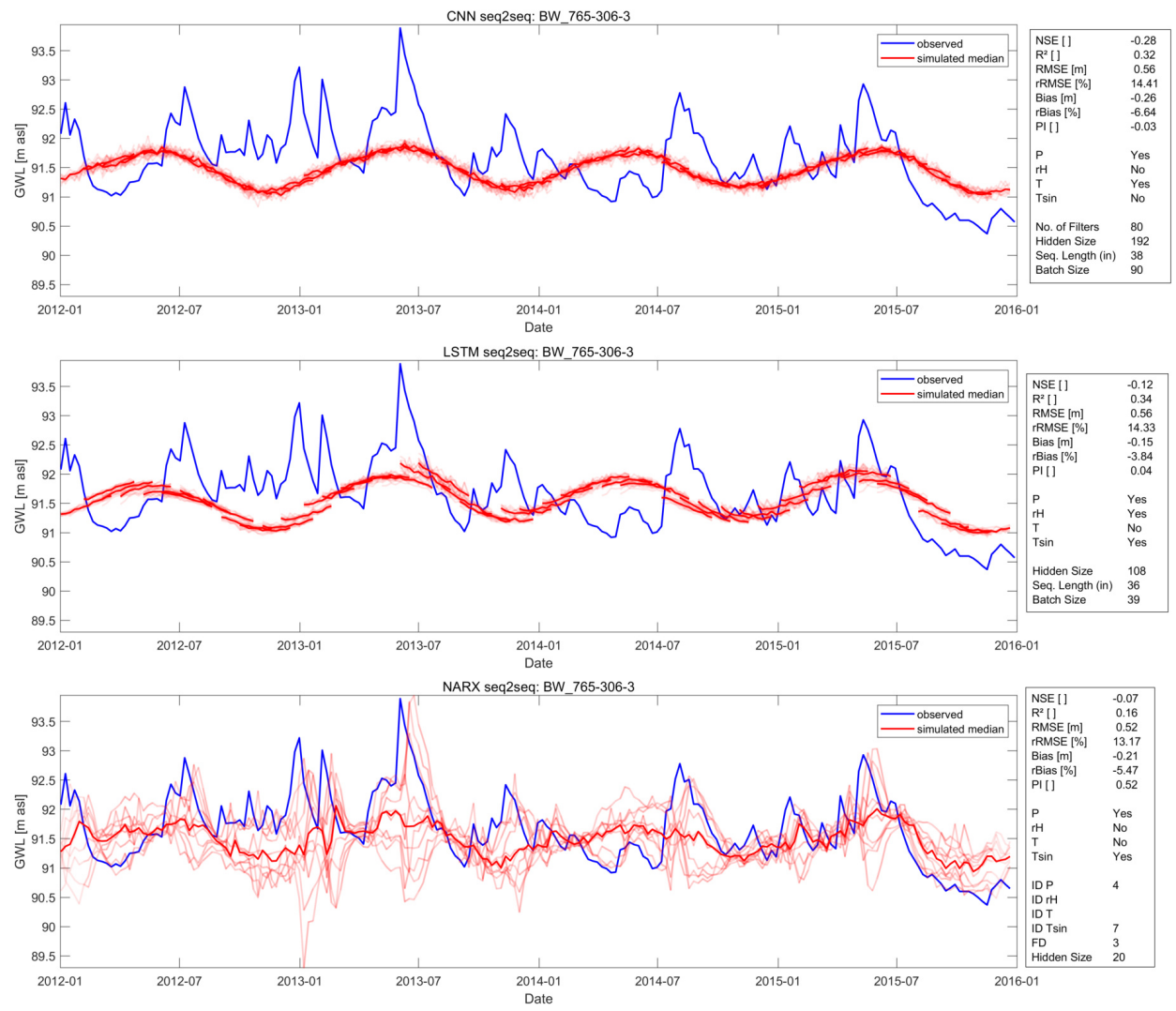

Figure S42: Seq2Seq test results for well BW_765-306-3 

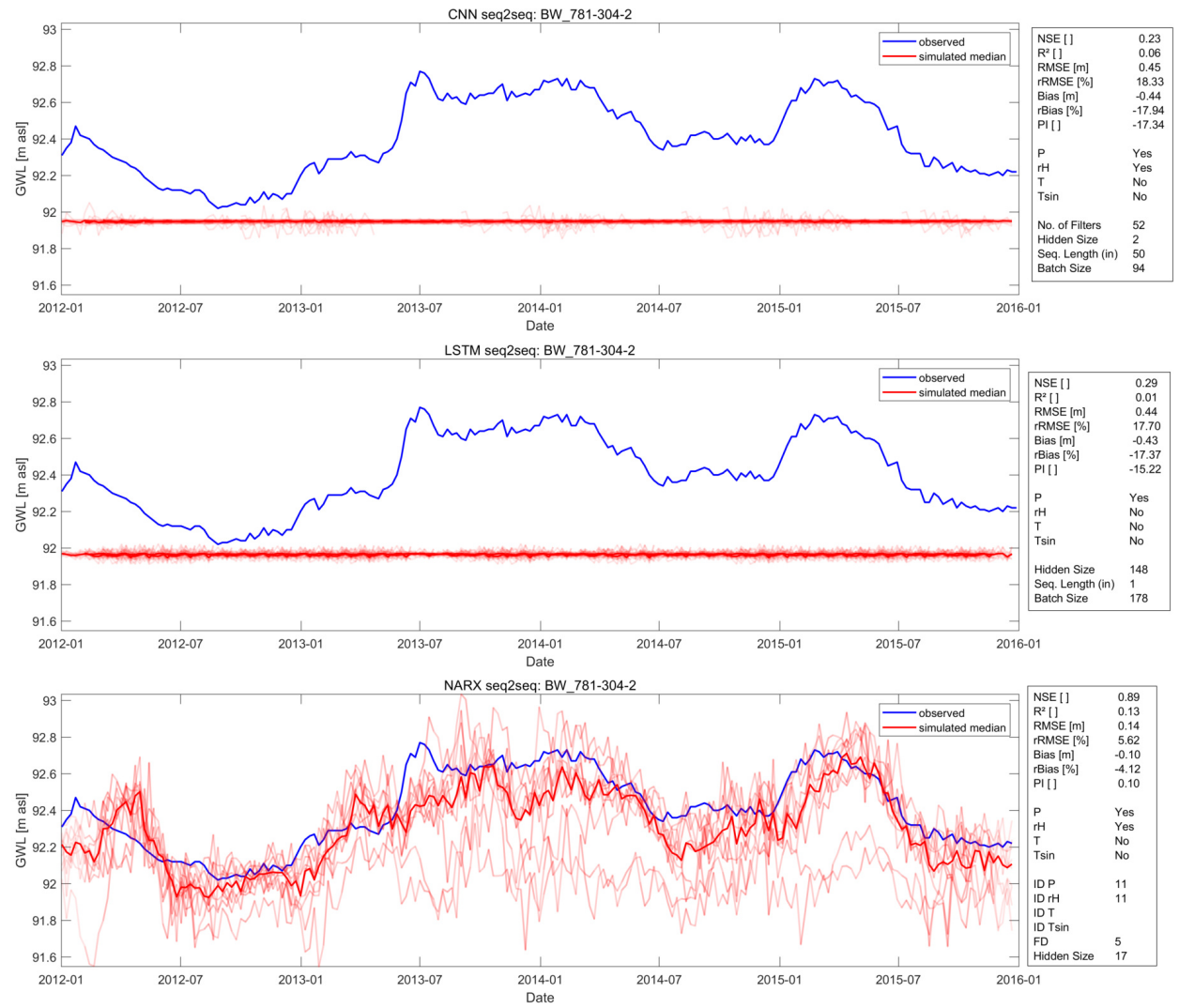

Figure S43: Seq2Seq test results for well BW_781-304-2 

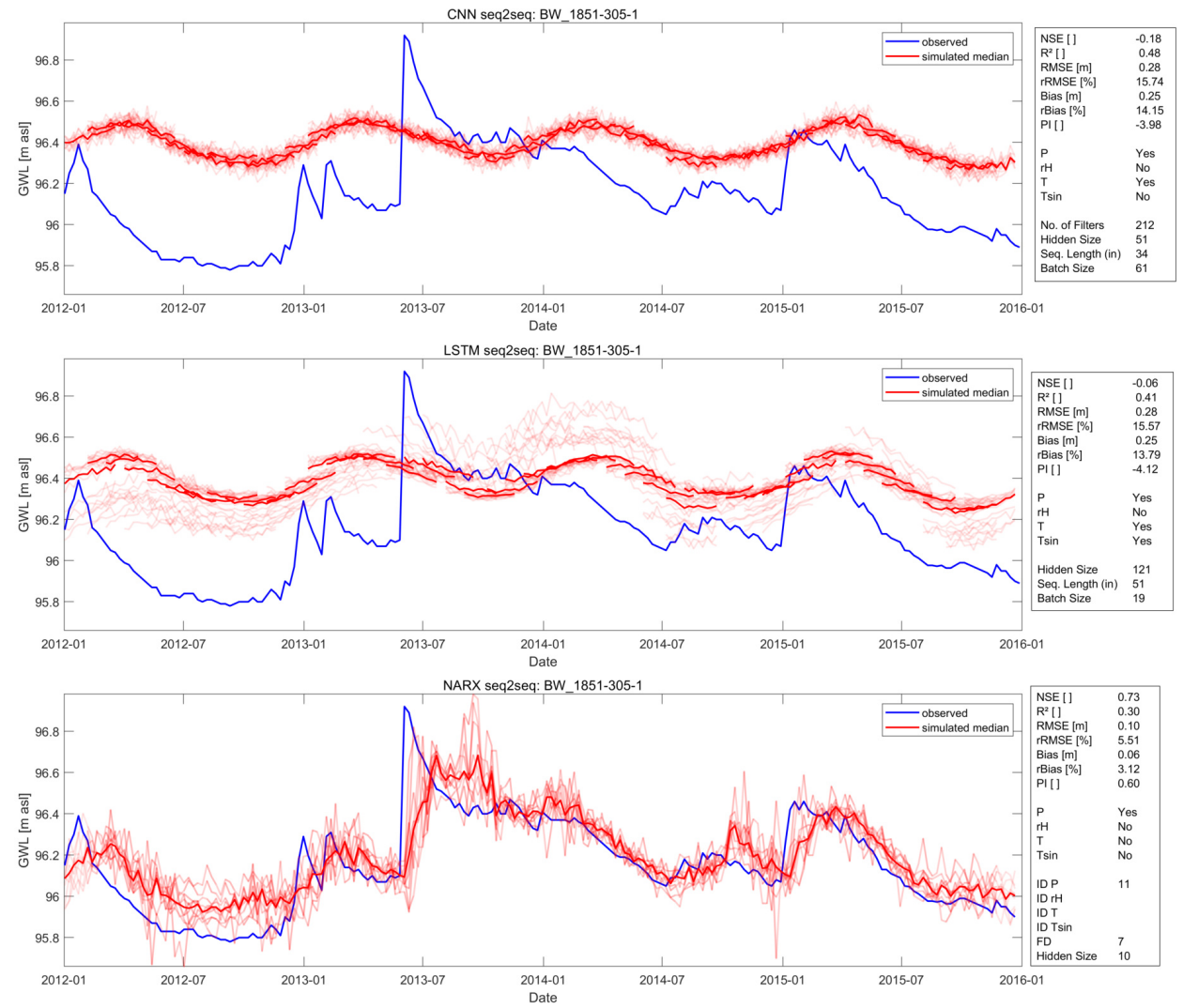

Figure S44: Seq2Seq test results for well BW_1851-305-1 

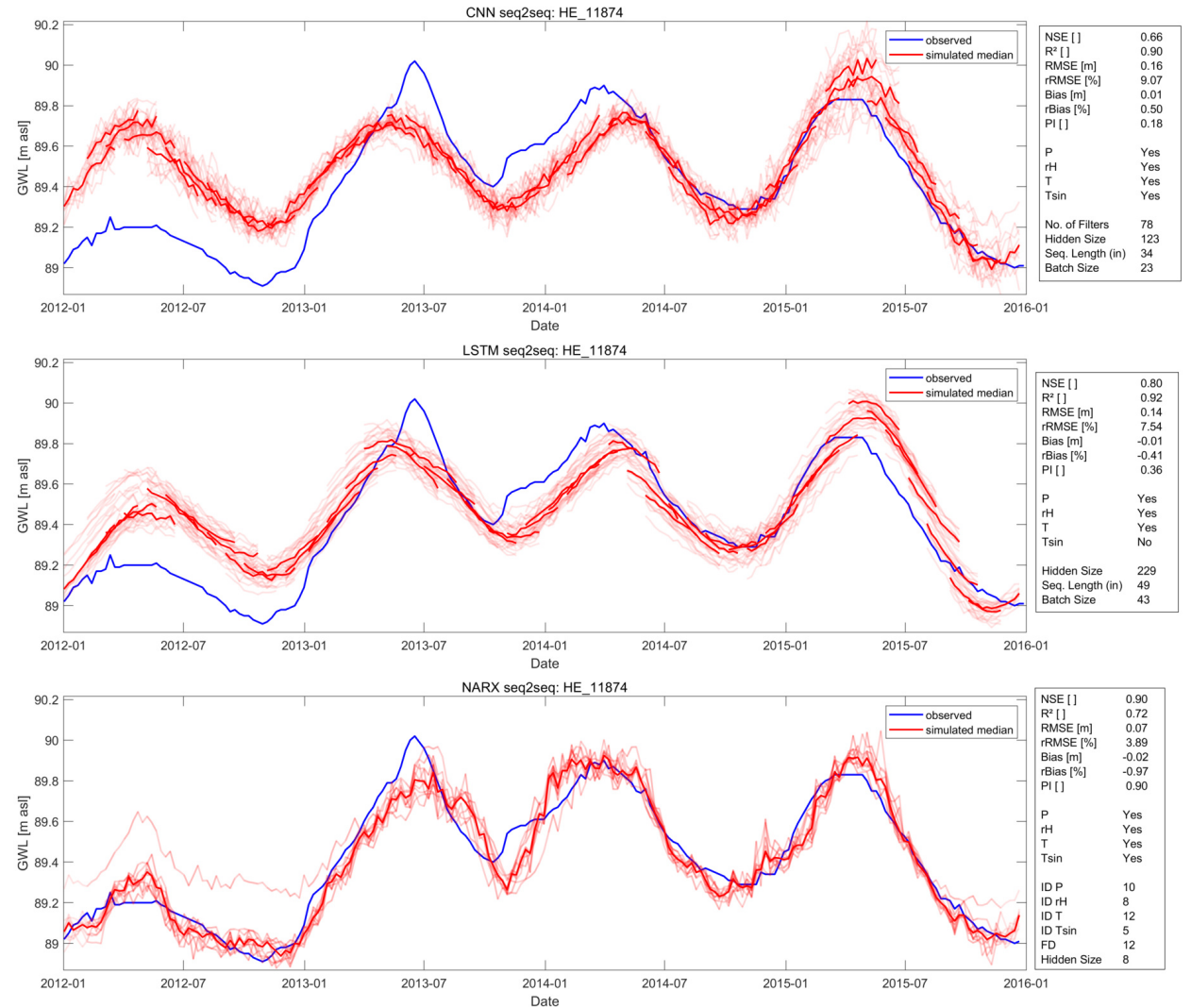

Figure S45: Seq2Seq test results for well HE_11874 

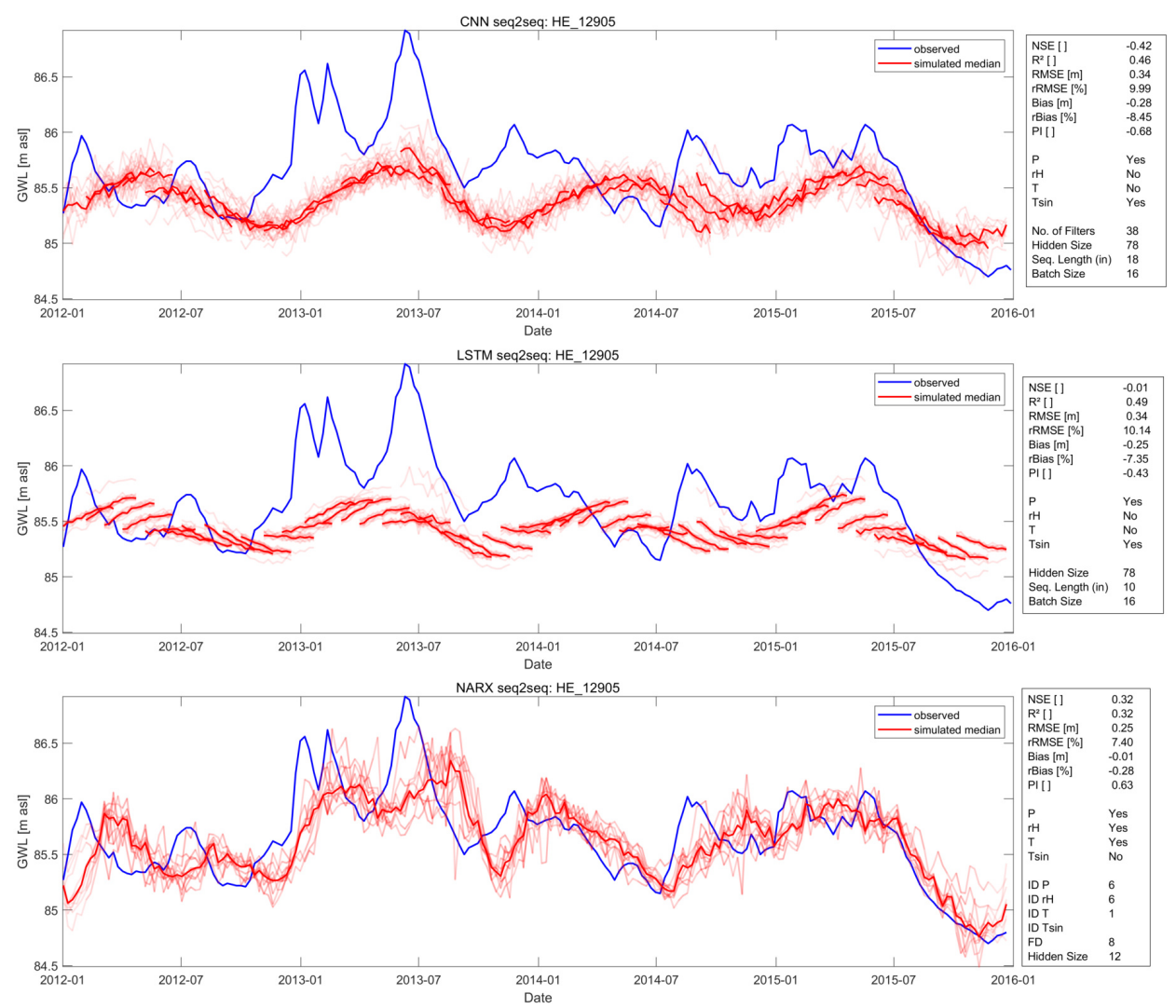

Figure S46: Seq2Seq test results for well HE_12905 

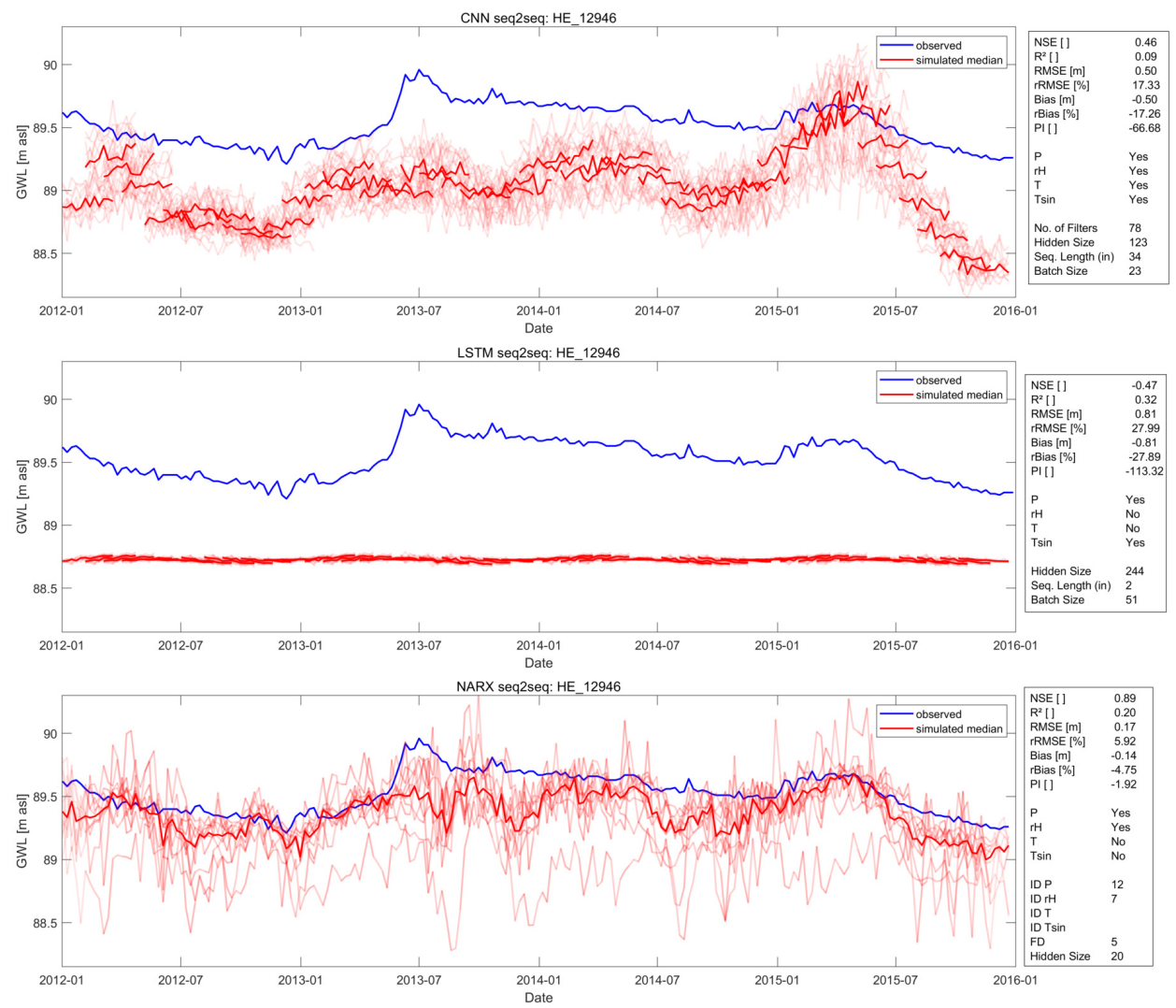

Figure S47: Seq2Seq test results for well HE_12946 

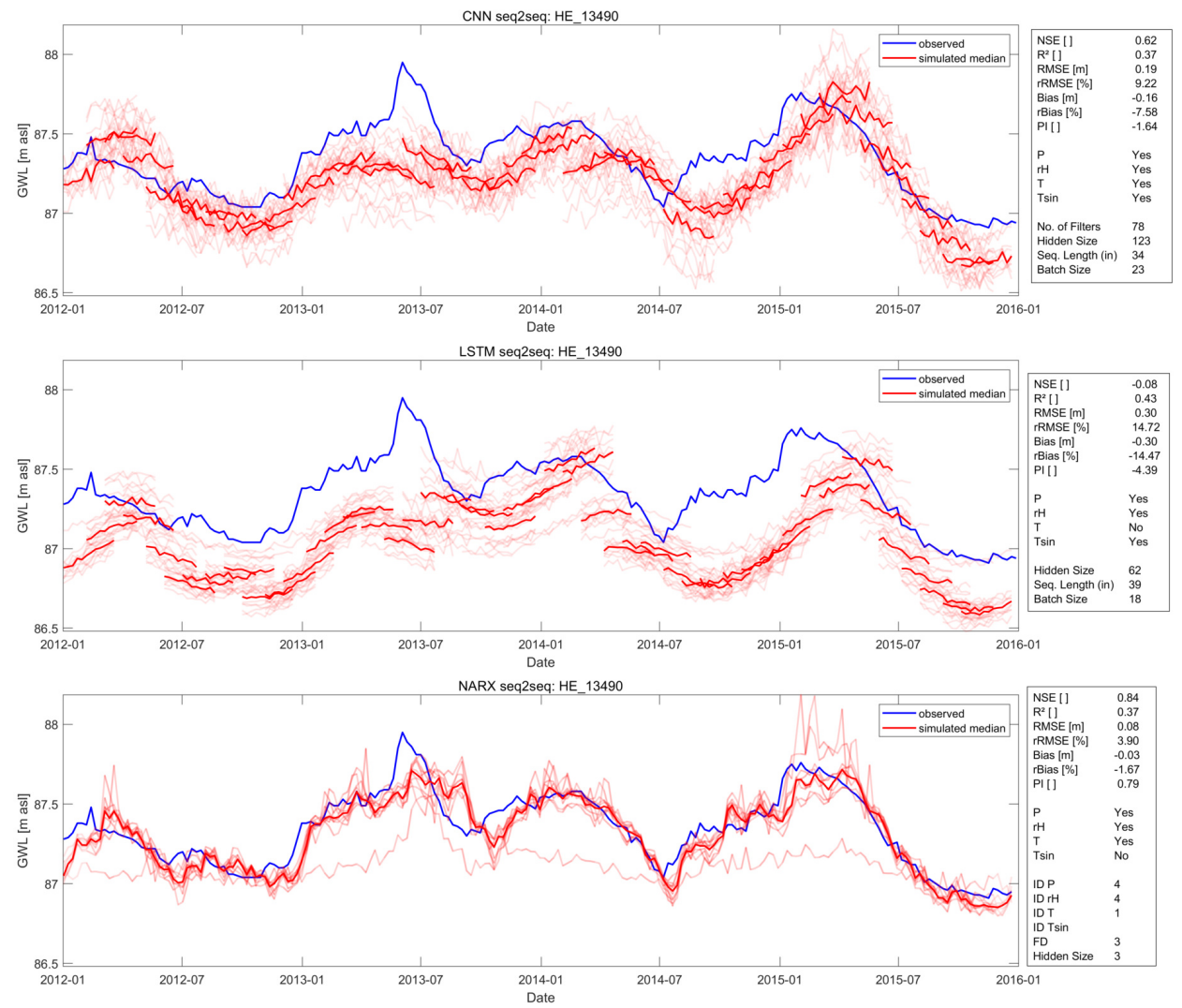

Figure S48: Seq2Seq test results for well HE_13490 

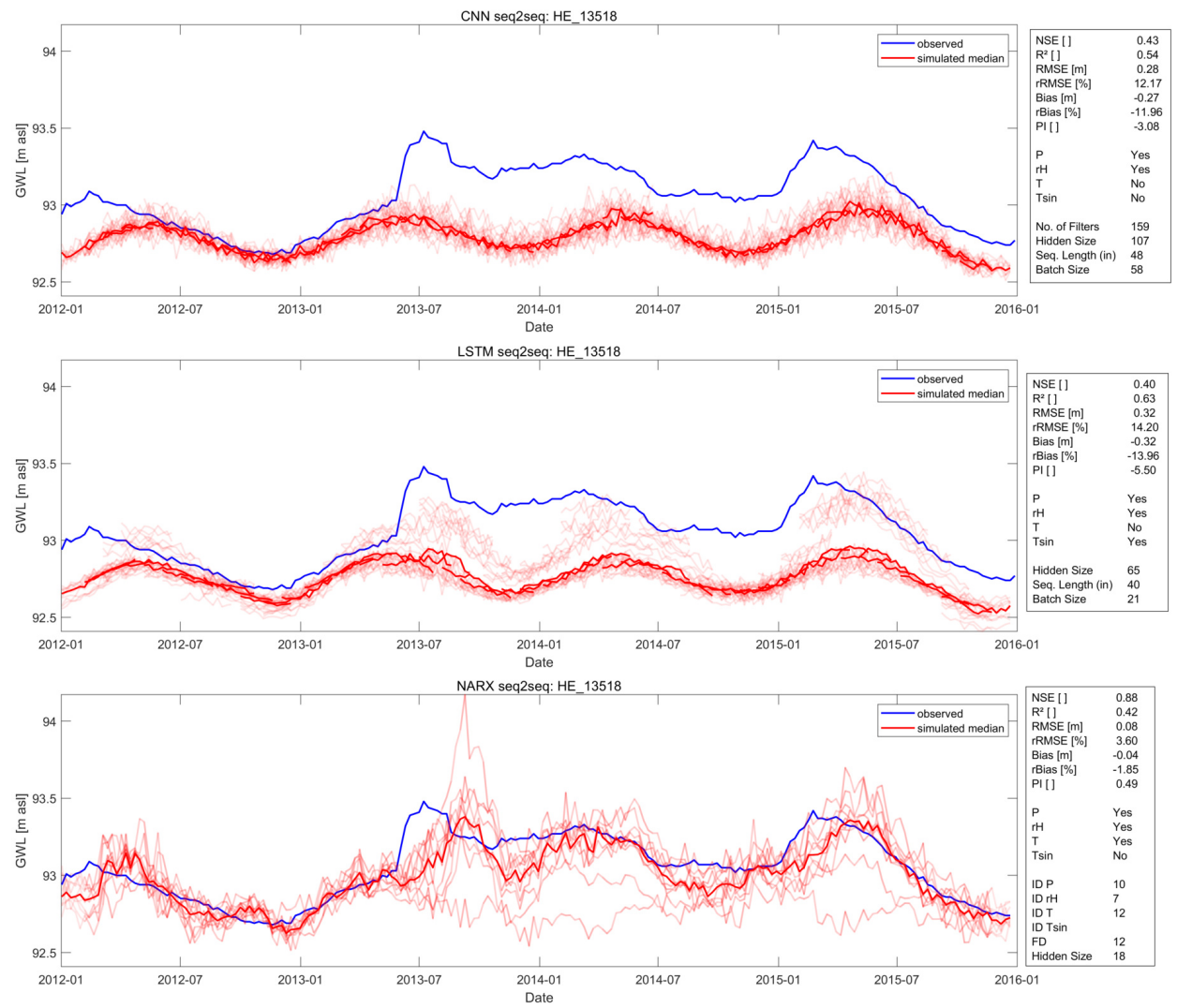

Figure S49: Seq2Seq test results for well HE_13518 

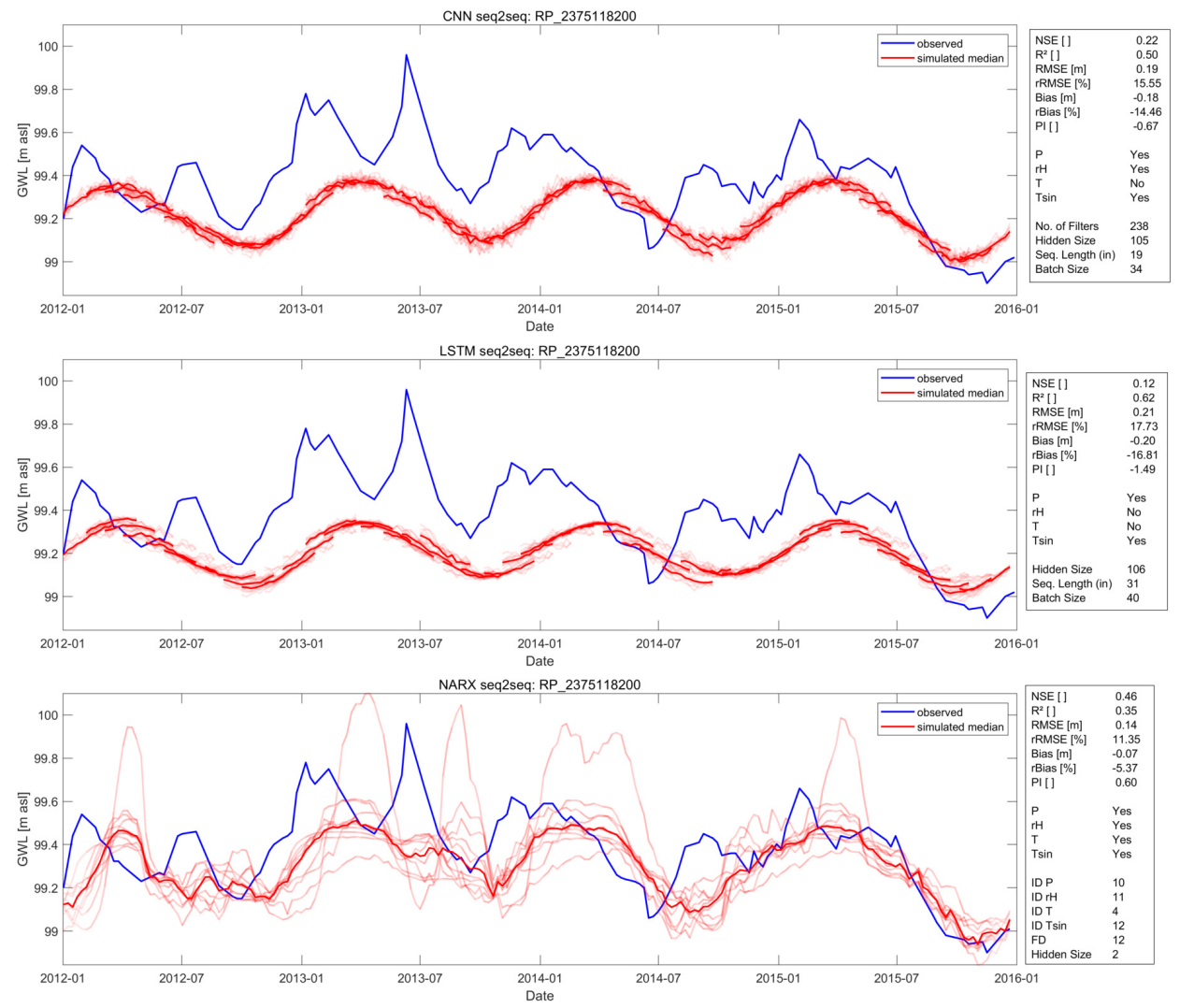

Figure S50: Seq2Seq test results for well RP_2375118200 

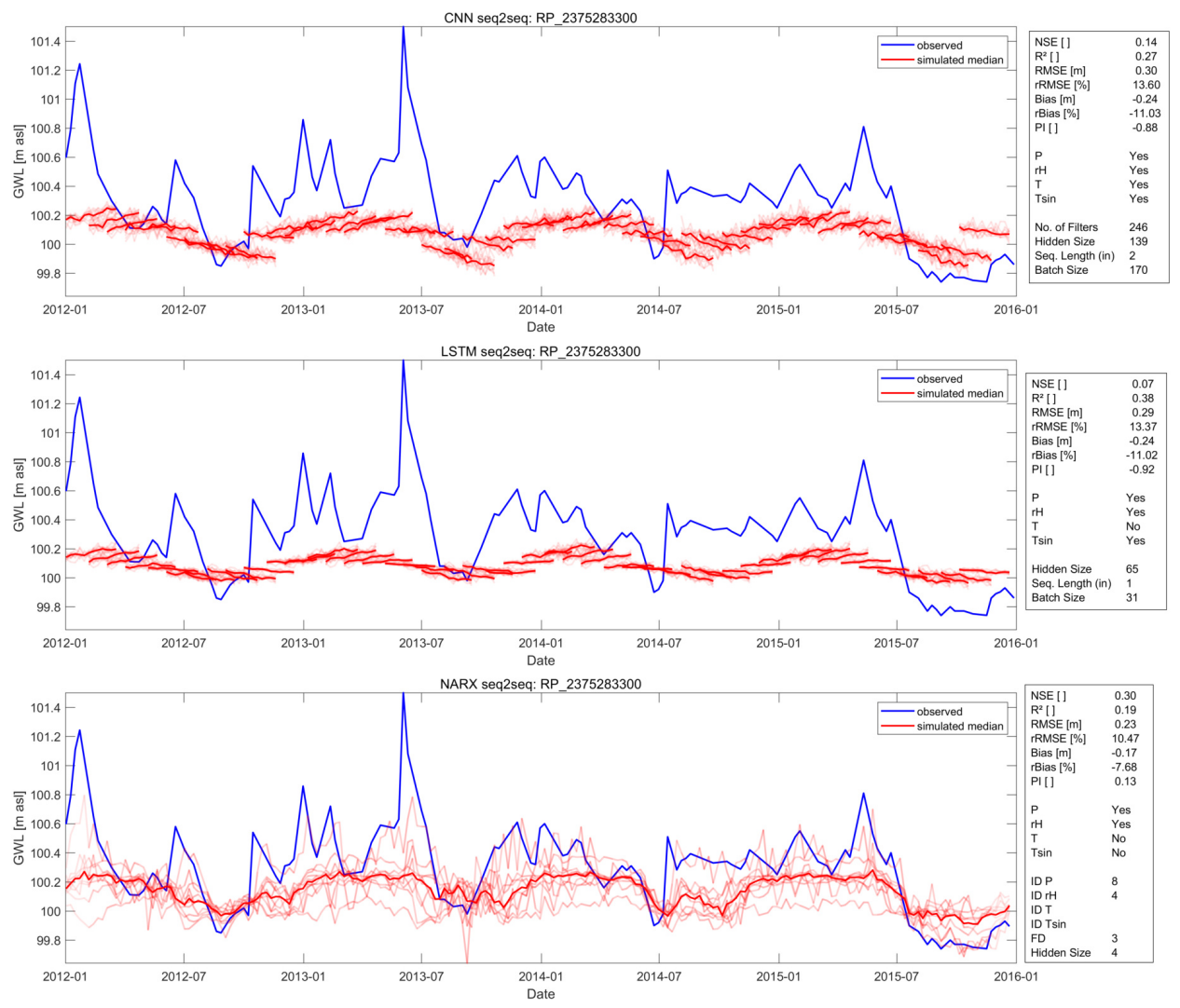

Figure S51: Seq2Seq test results for well RP_2375283300 

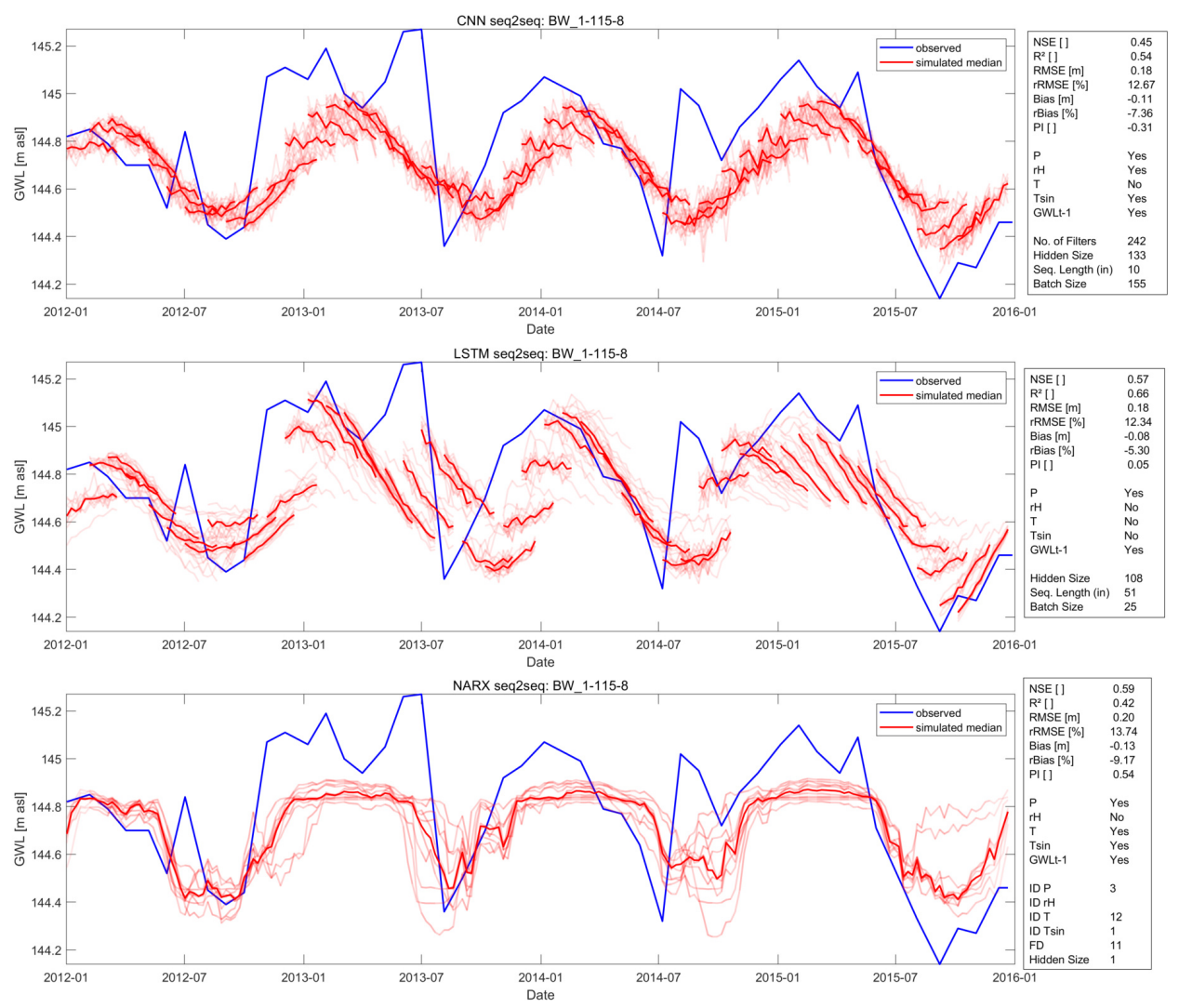

Figure S52: Seq2Seq $\mathrm{GWL}_{t-1}$ test results for well BW_1-115-8 

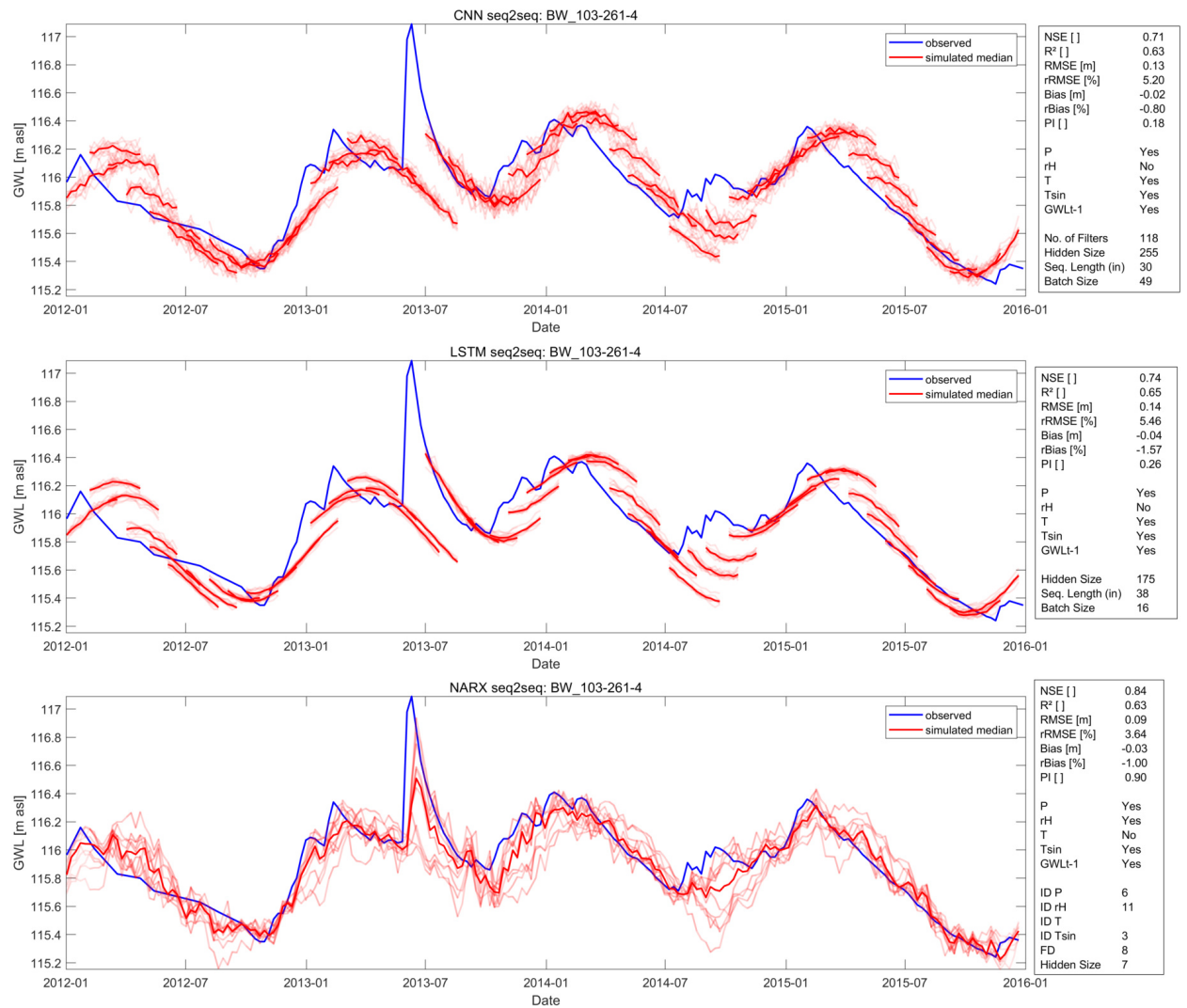

Figure S53: Seq2Seq $\mathrm{GWL}_{t-1}$ test results for well BW_103-261-4 

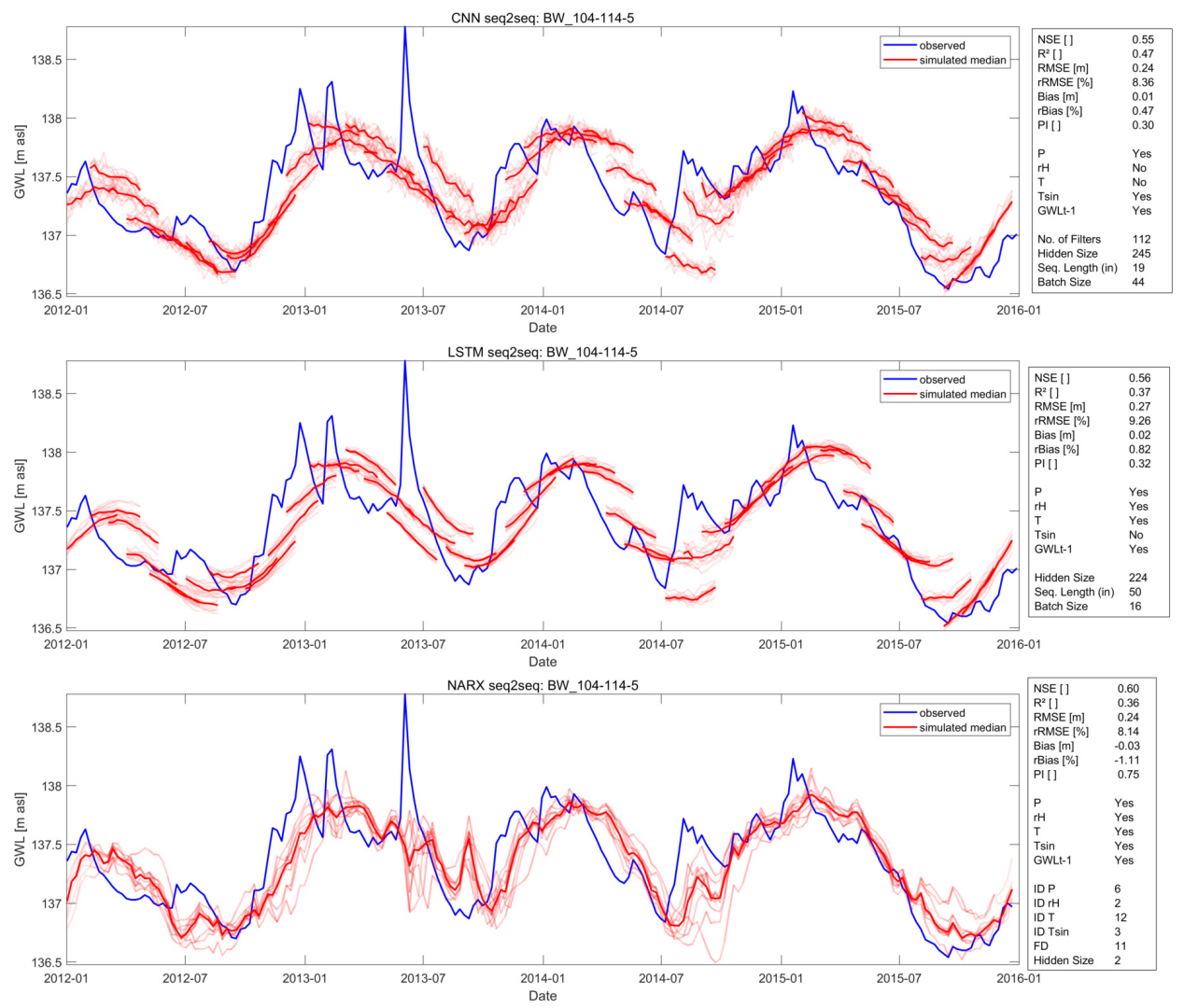

Figure S54: Seq2Seq GWL G-1 $_{t}$ test results for well BW_104-114-5 

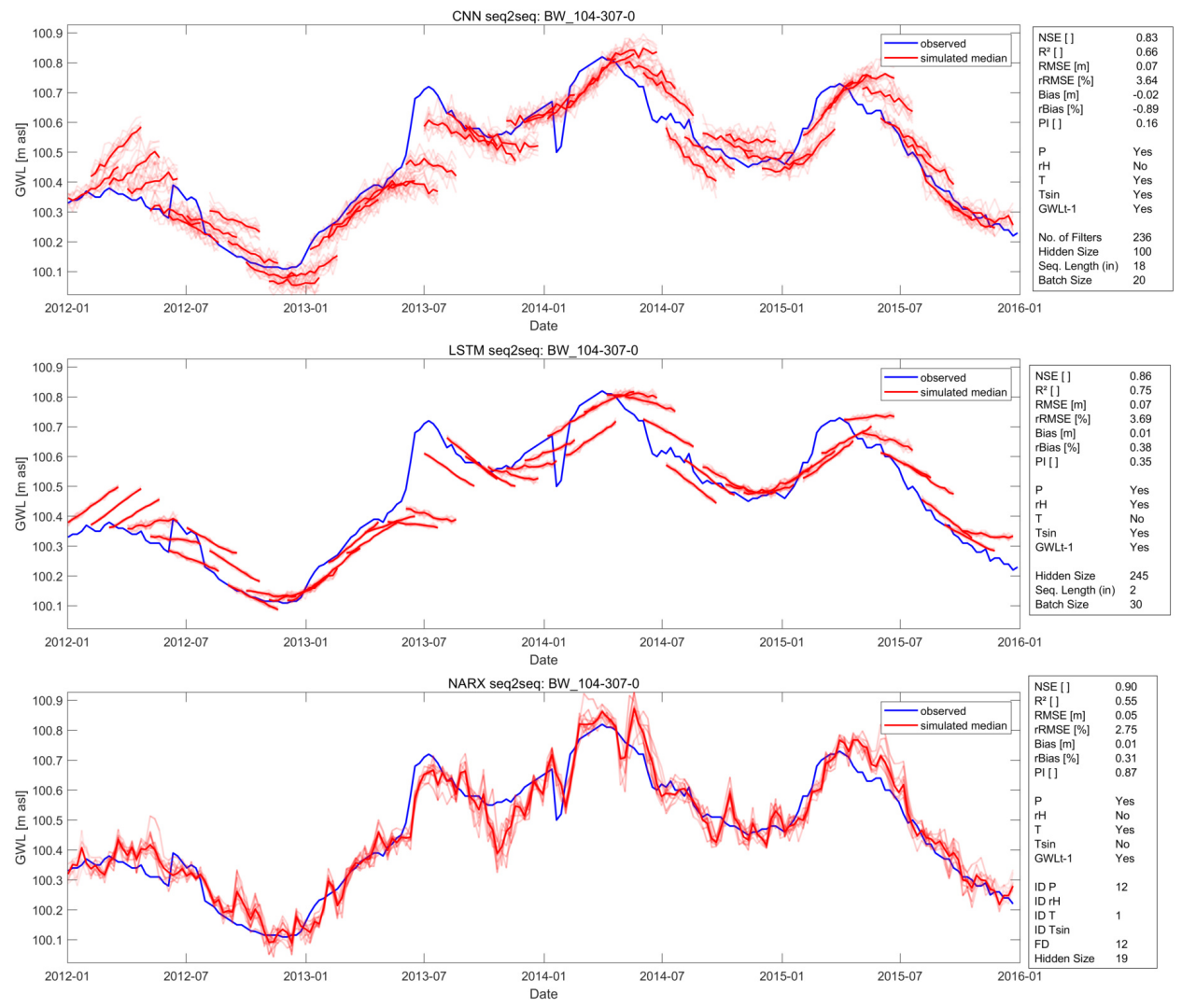

Figure S55: Seq2Seq GWL GL1 $_{t}$ test results for well BW_104-307-0 

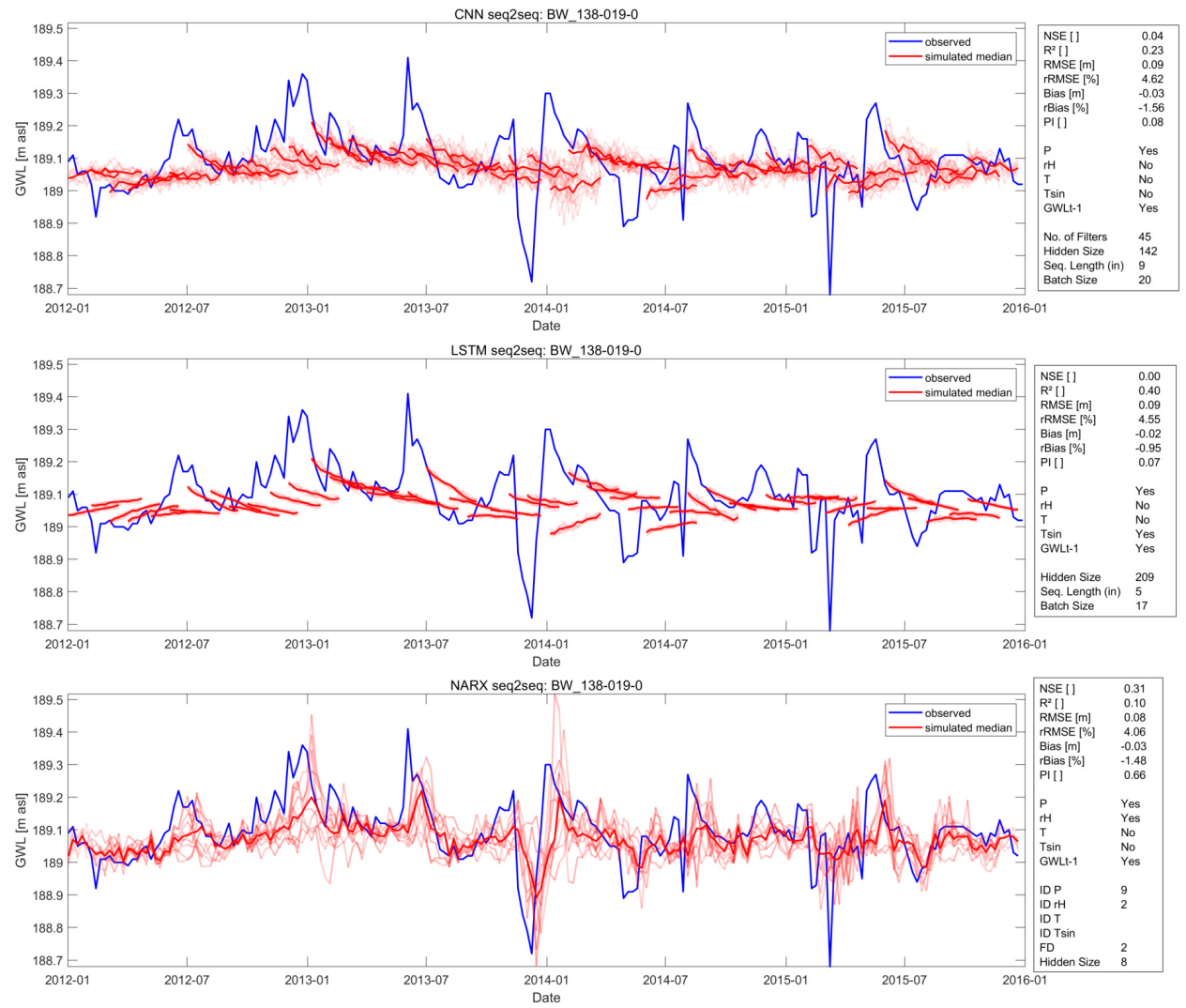

Figure S56: Seq2Seq GWL G-1 $_{t}$ test results for well BW_138-019-0 

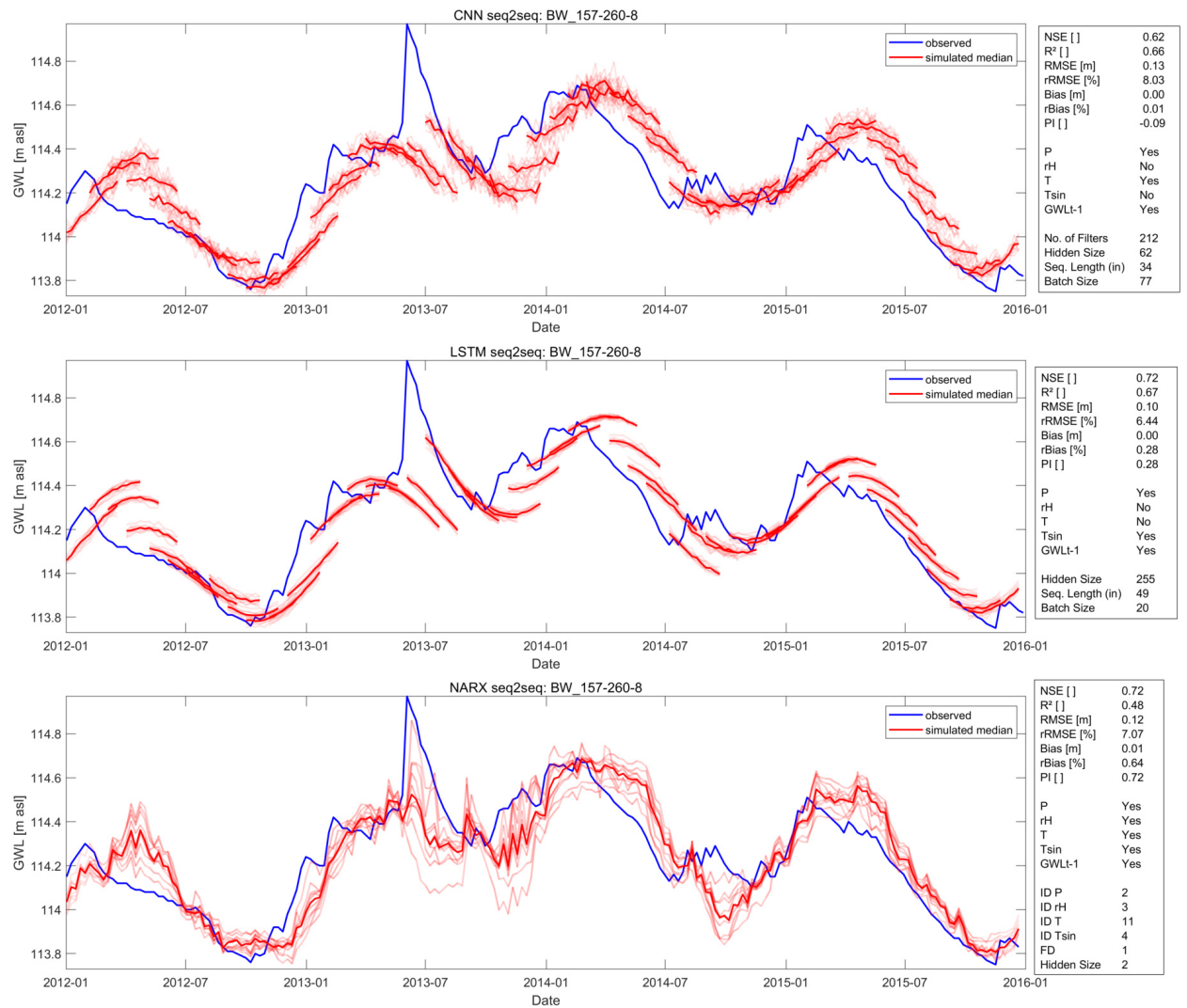

Figure S57: Seq2Seq GWL $\mathrm{GL}_{t-1}$ test results for well BW_157-260-8 

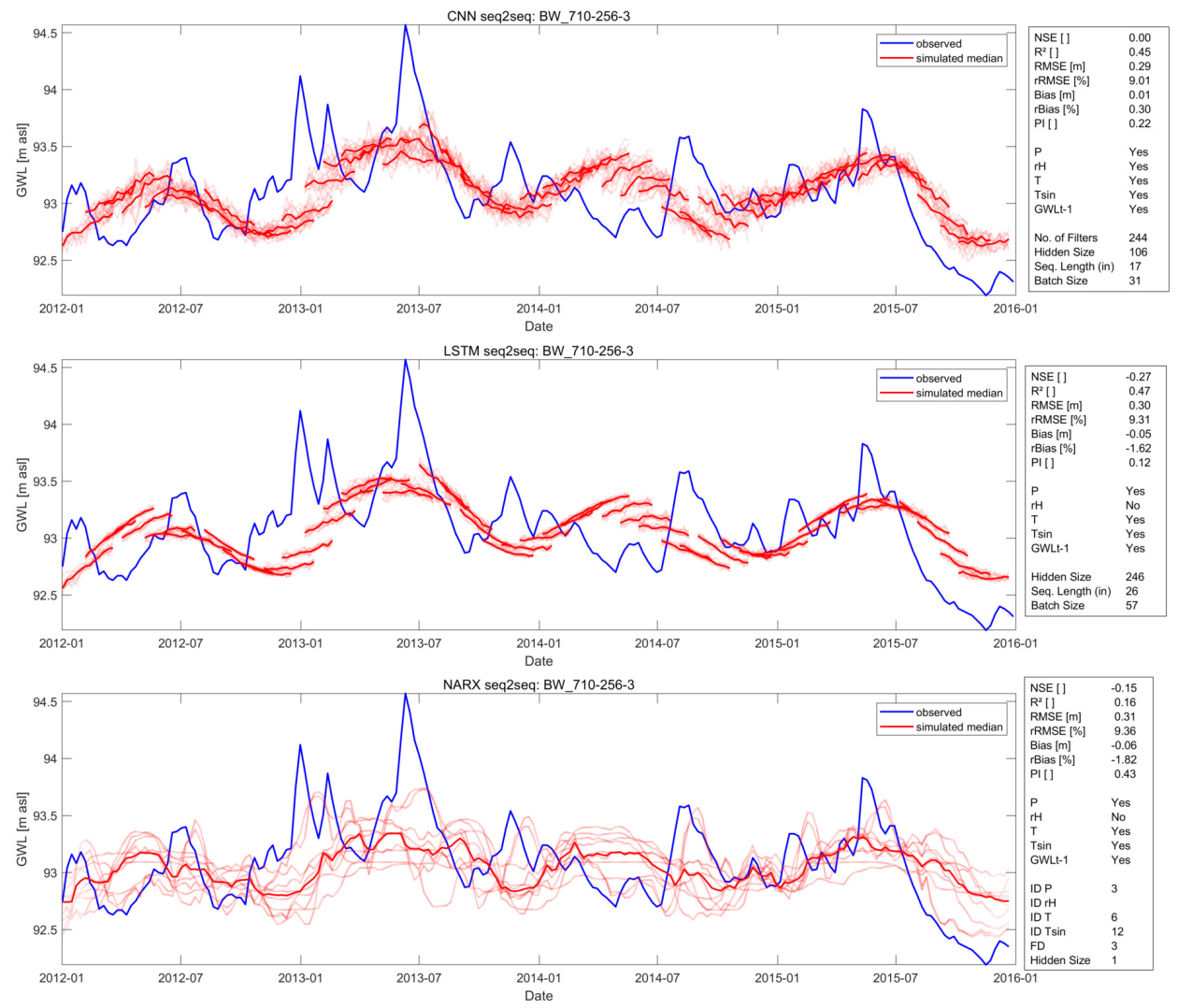

Figure S58: Seq2Seq $\mathrm{GWL}_{t-1}$ test results for well BW_710-256-3 

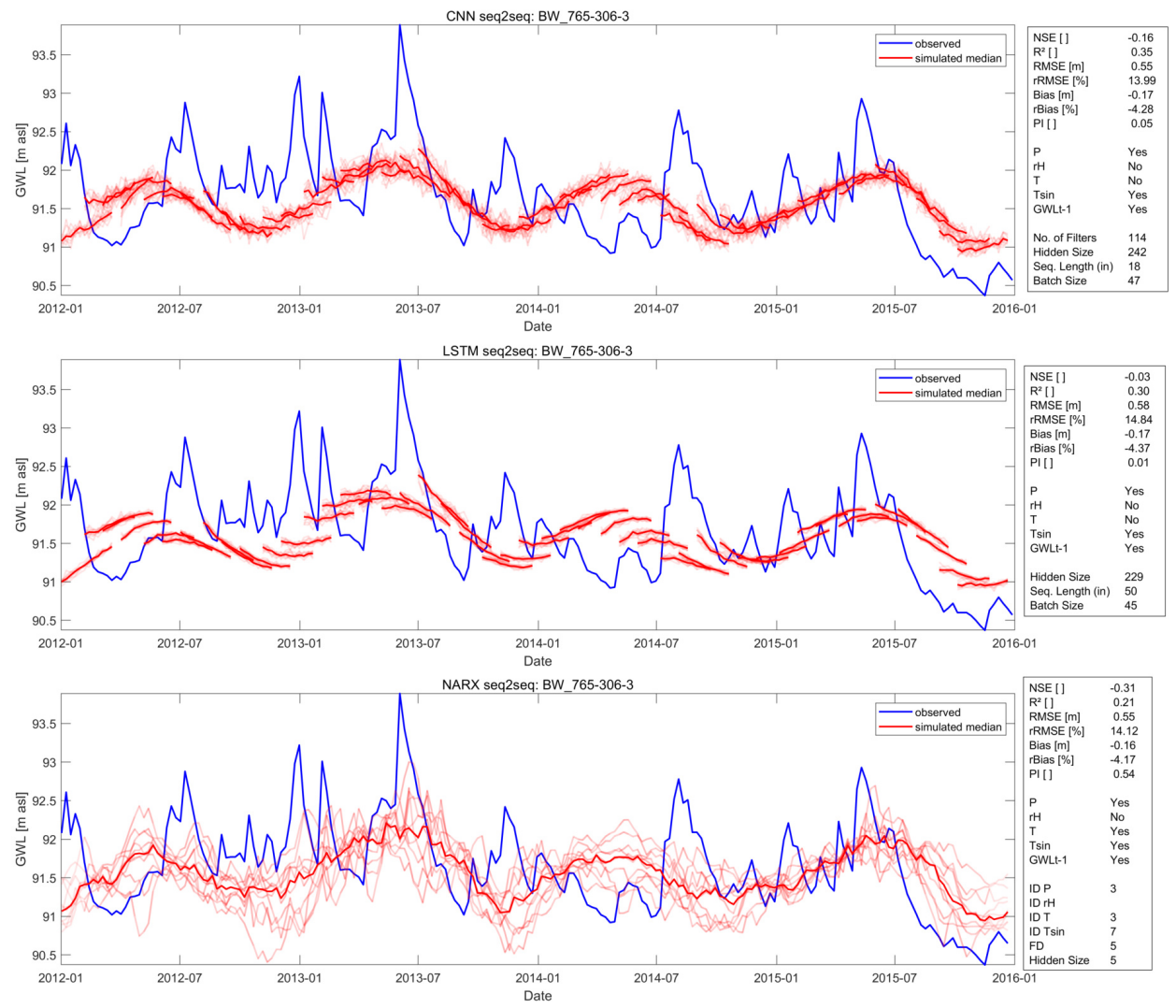

Figure S59: Seq2Seq GWL $\mathrm{GW}_{t-1}$ test results for well BW_765-306-3 

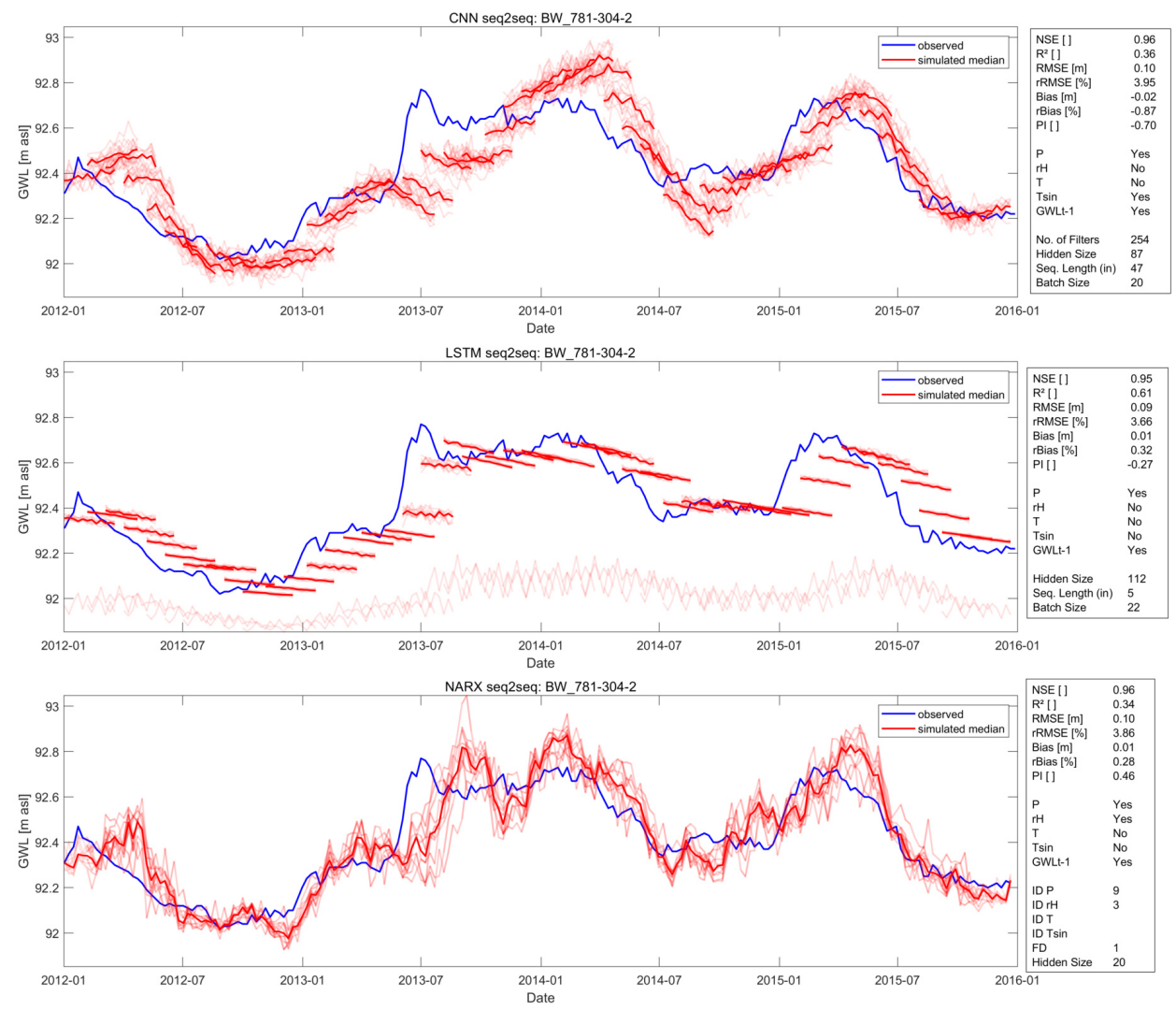

Figure S60: Seq2Seq $\mathrm{GWL}_{t-1}$ test results for well BW_781-304-2 

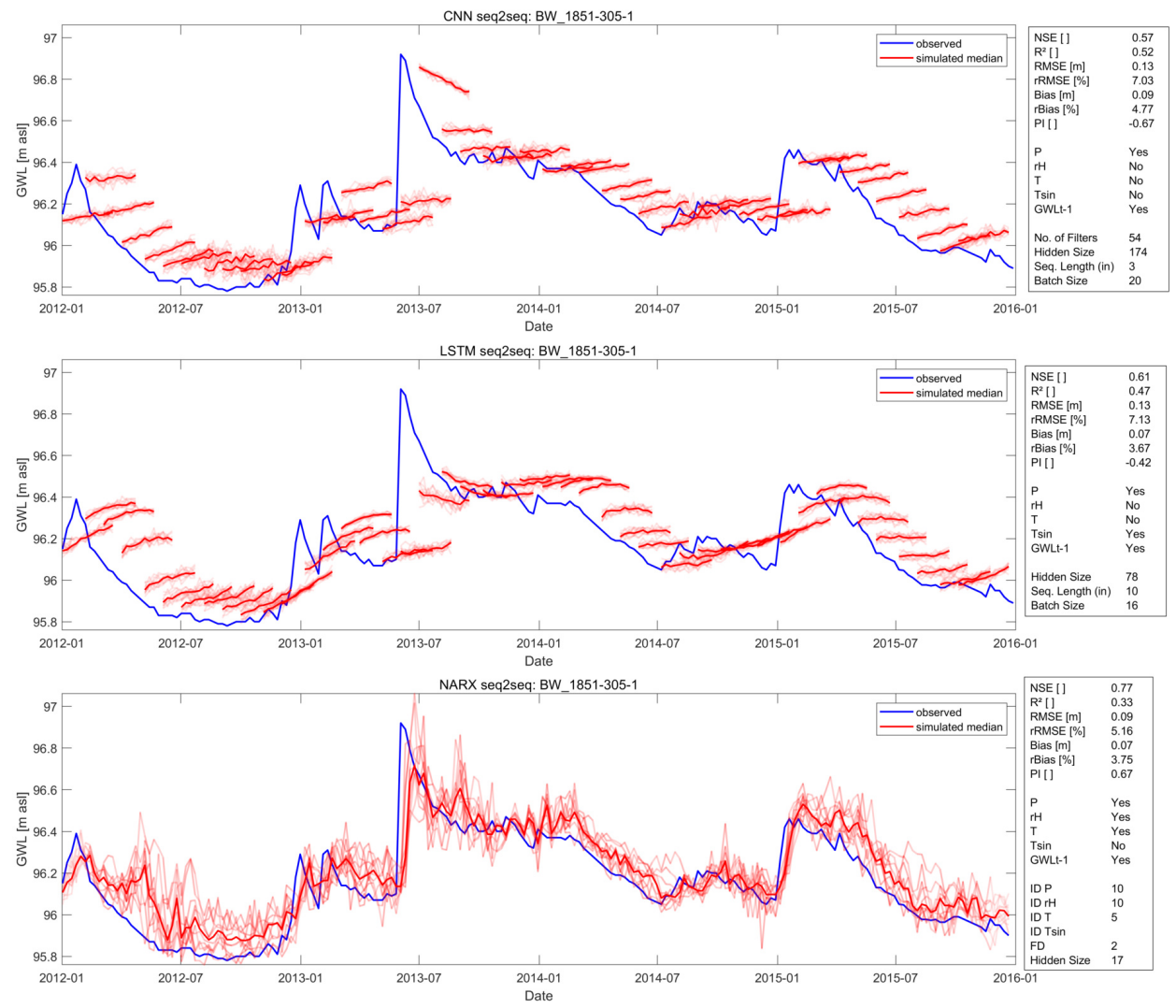

Figure S61: Seq2Seq $\mathrm{GWL}_{t-1}$ test results for well BW_1851-305-1 

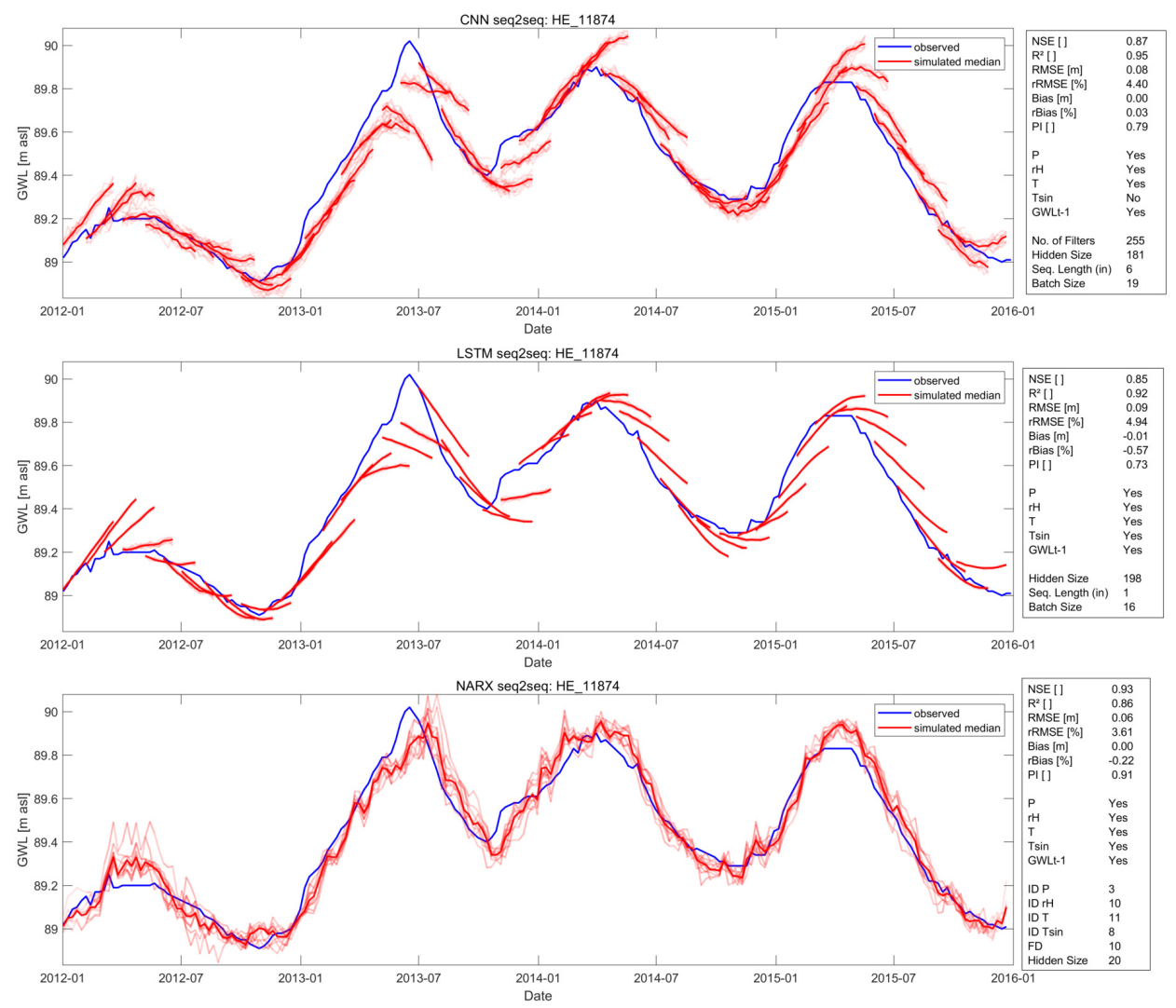

Figure S62: Seq2Seq GWL G $_{t-1}$ test results for well HE_11874 

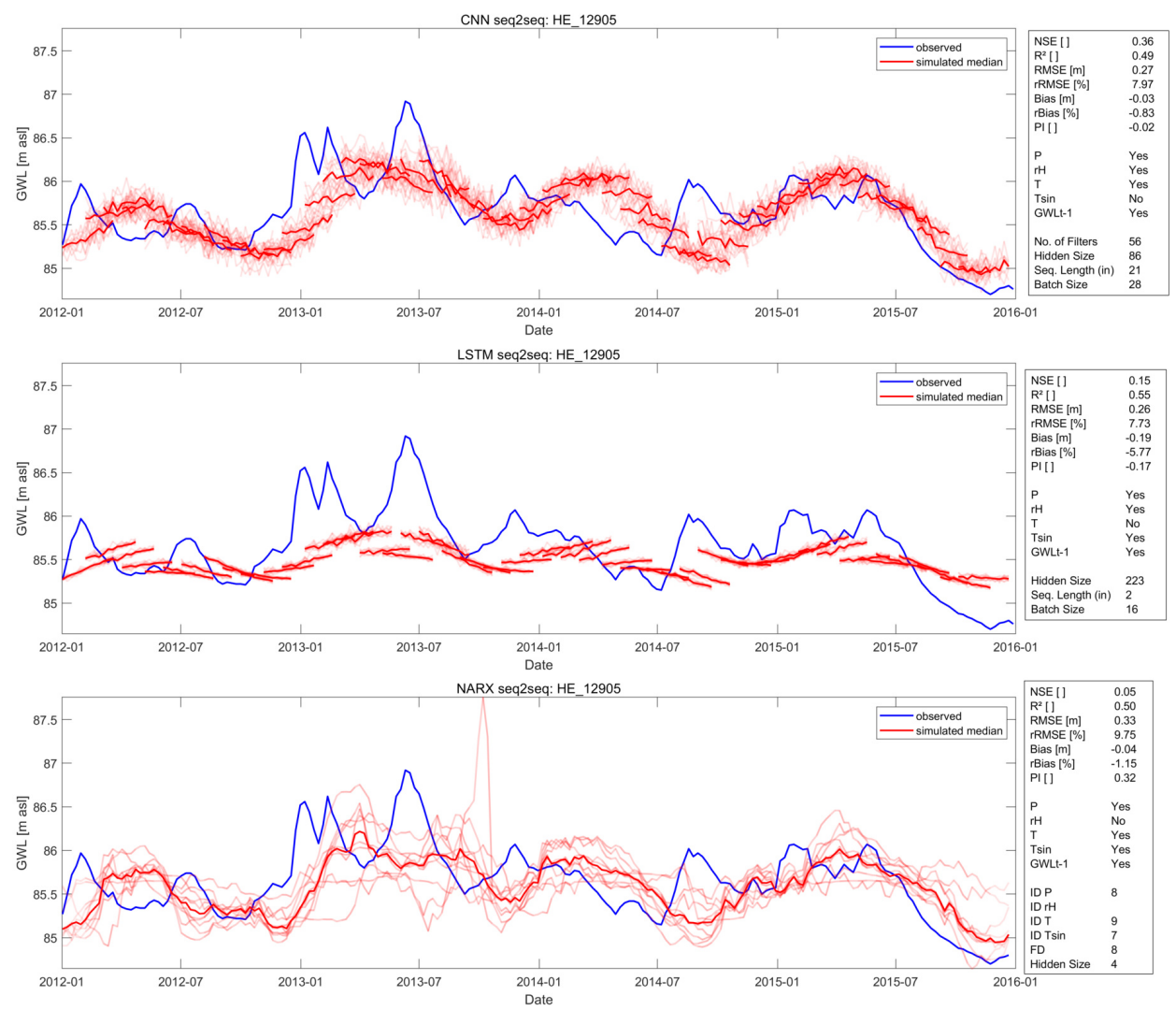

Figure S63: Seq2Seq GWL $\mathrm{G}_{t-1}$ test results for well HE_12905 

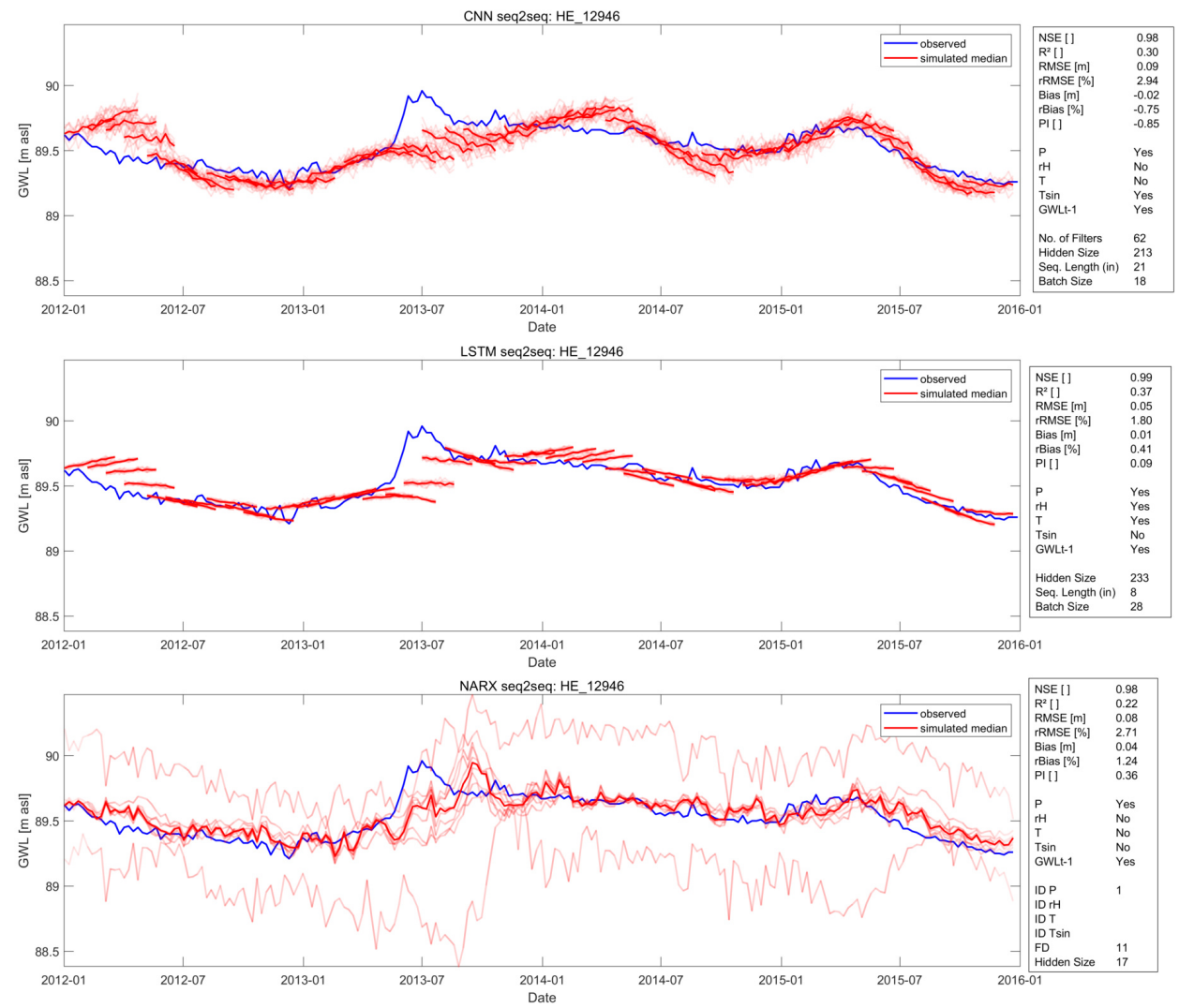

Figure S64: Seq2Seq GWL G-1 $_{t-1}$ test results for well HE_12946 

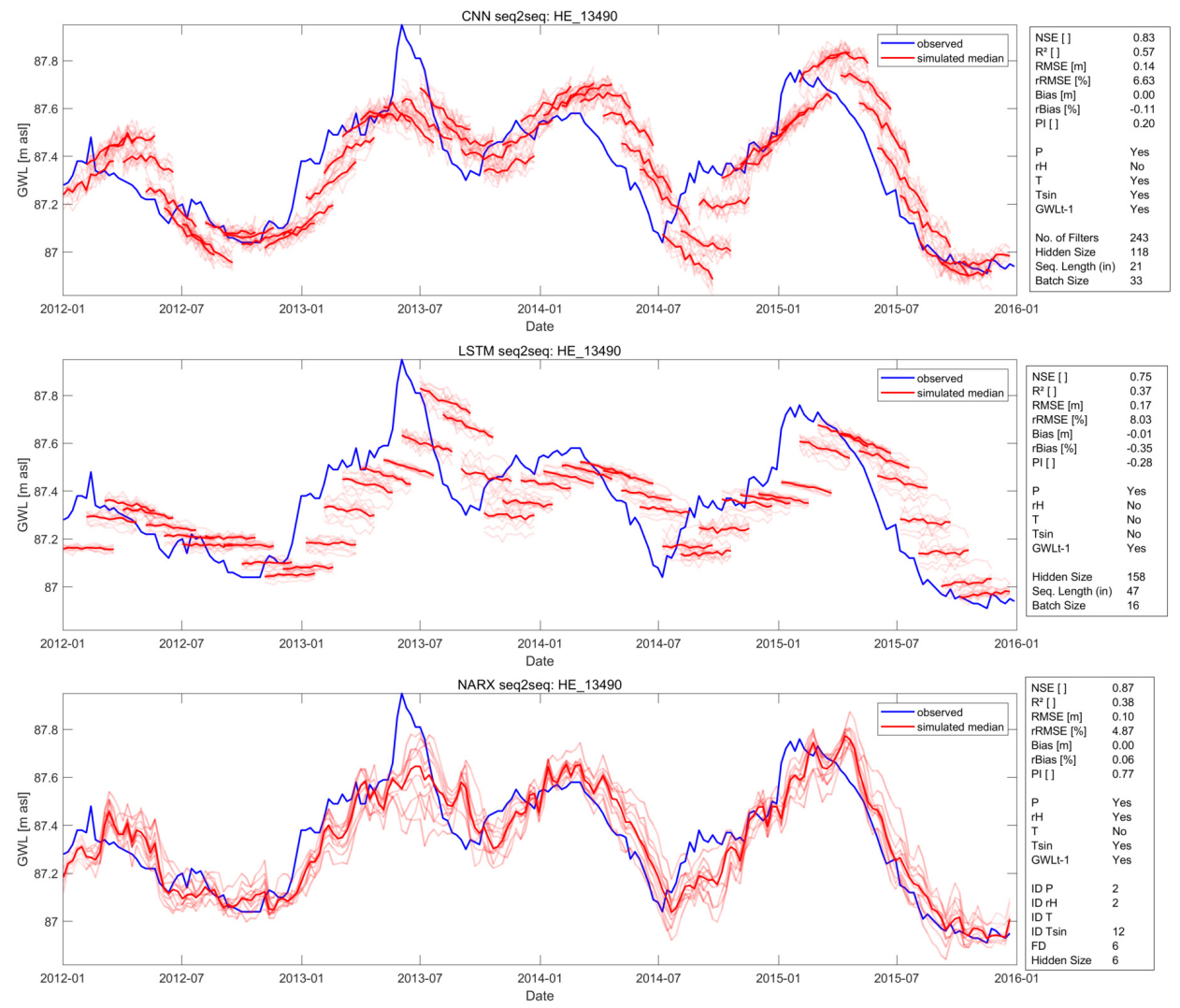

Figure S65: Seq2Seq GWL t-1 $_{1}$ test results for well HE_13490 

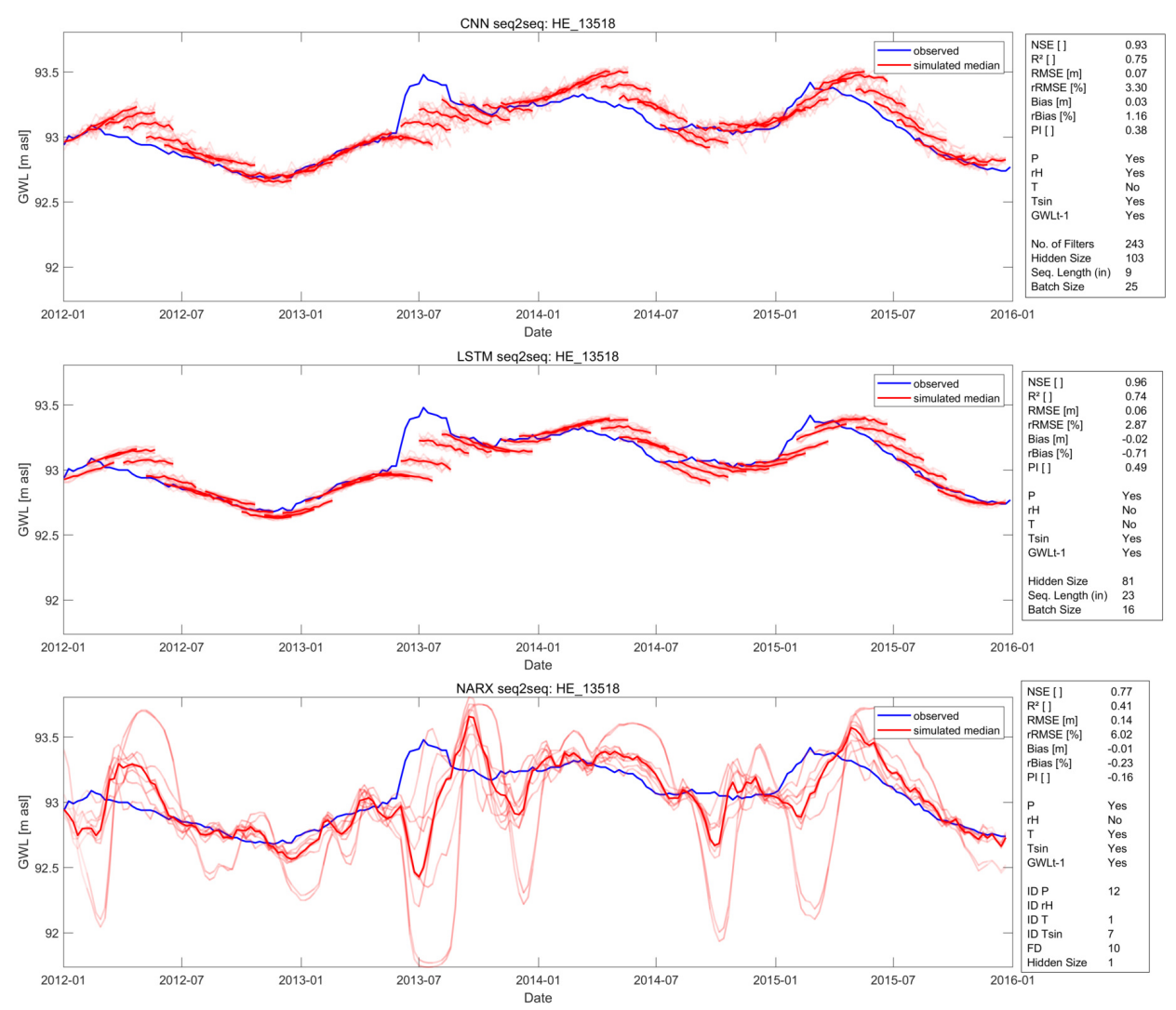

Figure S66: Seq2Seq GWL $\mathrm{G}_{t-1}$ test results for well HE_13518 

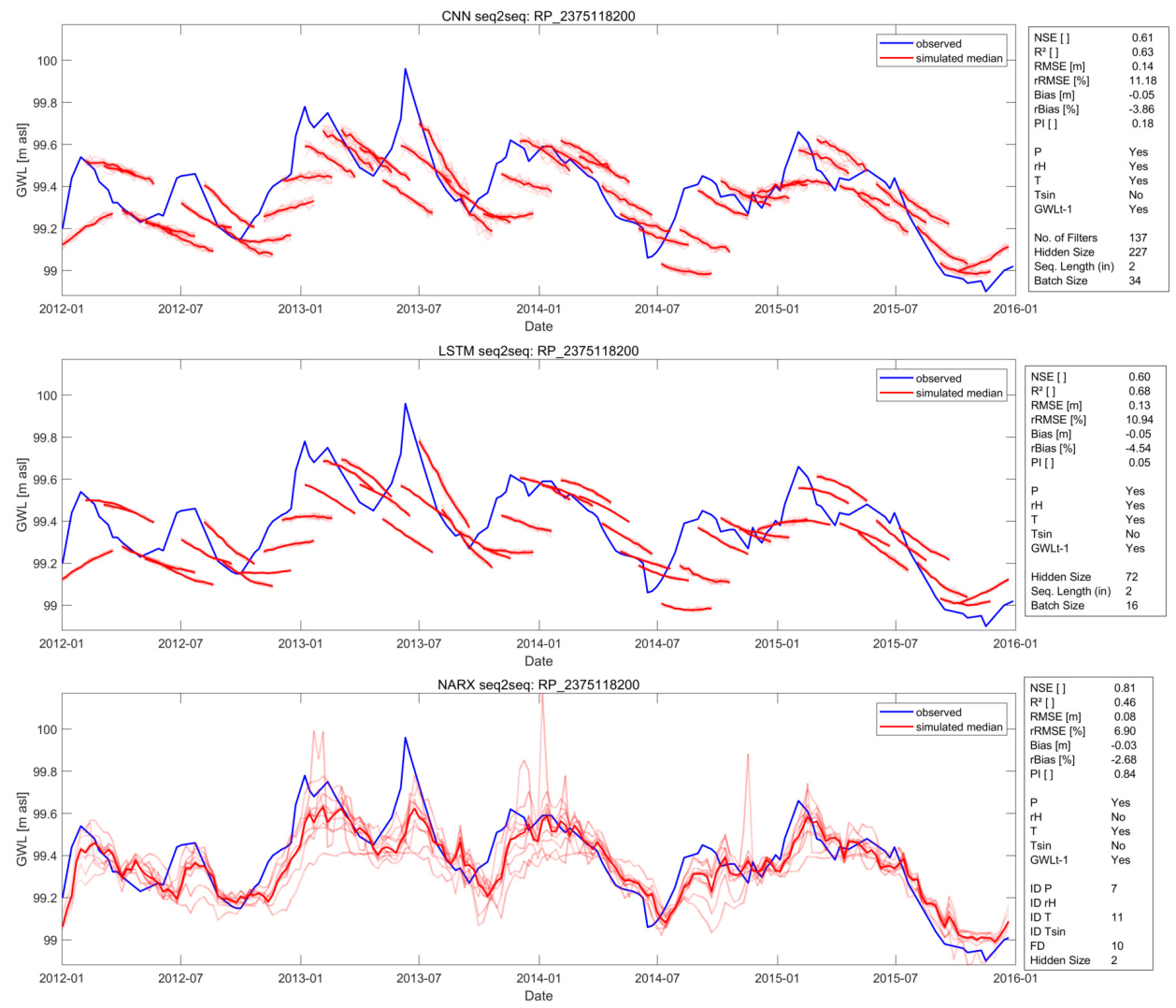

Figure S67: Seq2Seq $\mathrm{GWL}_{t-1}$ test results for well RP_2375118200 

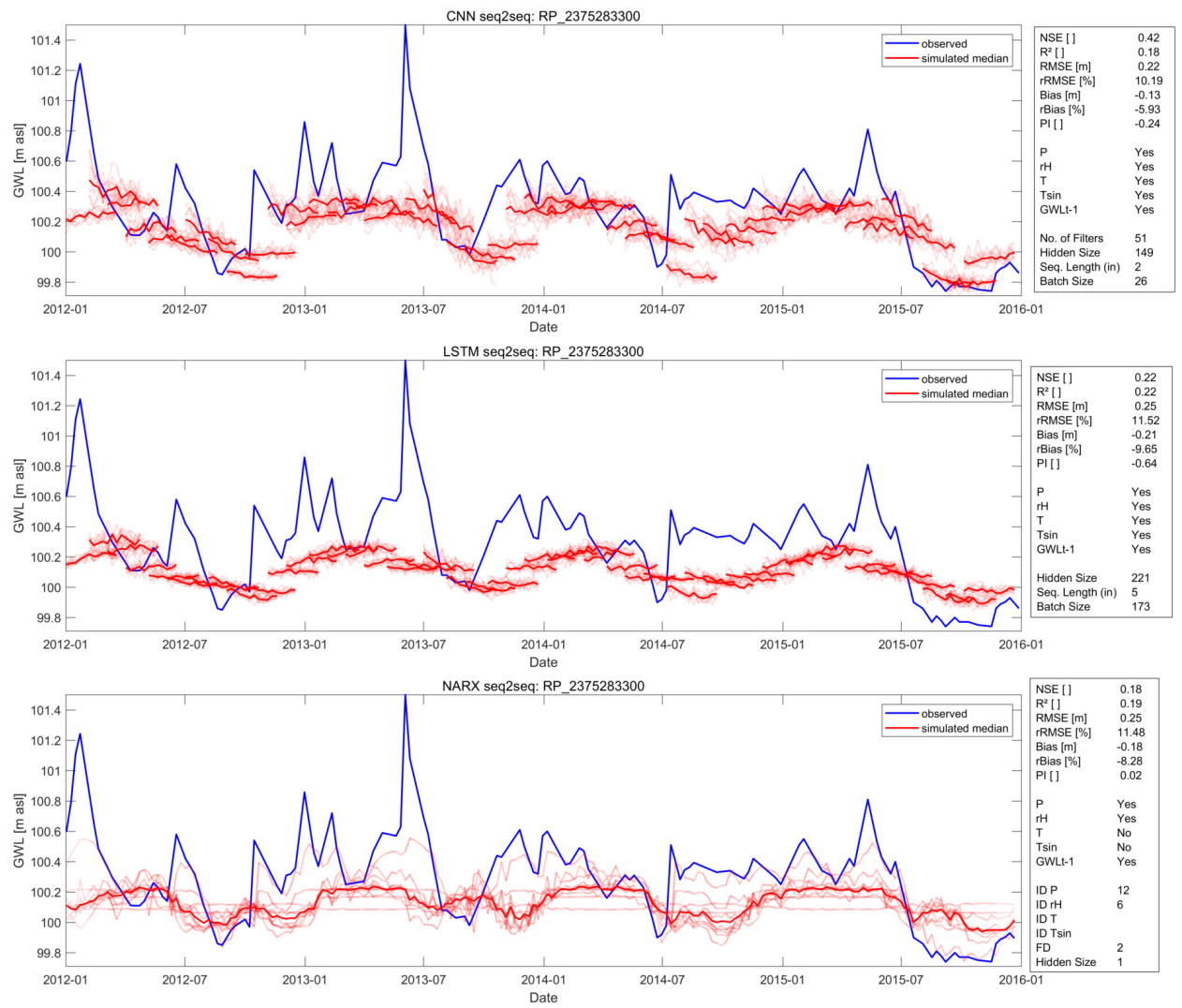

Figure S68: Seq2Seq $\mathrm{GWL}_{t-1}$ test results for well RP_2375283300 


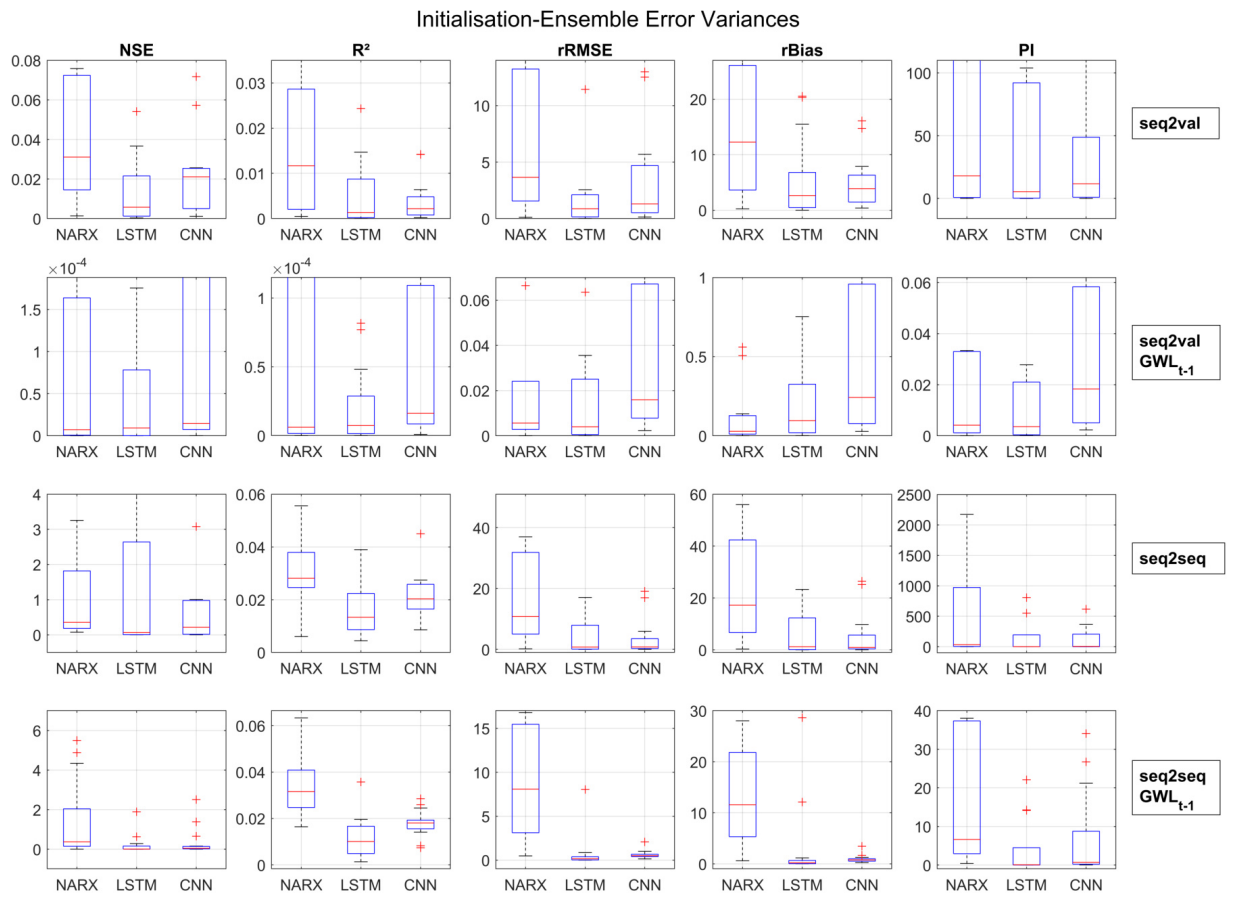

Figure S69: Error Variance of Initialization Ensemble Members 

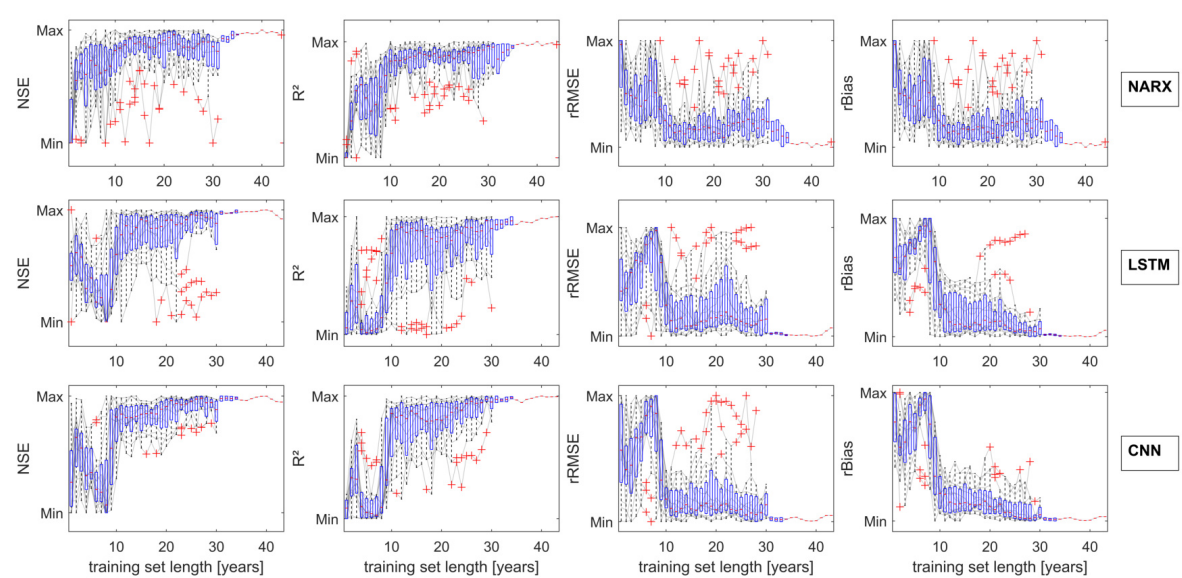

Figure S70: Influence of the available amount of training data on seq2val model performance based on meteorological inputs.
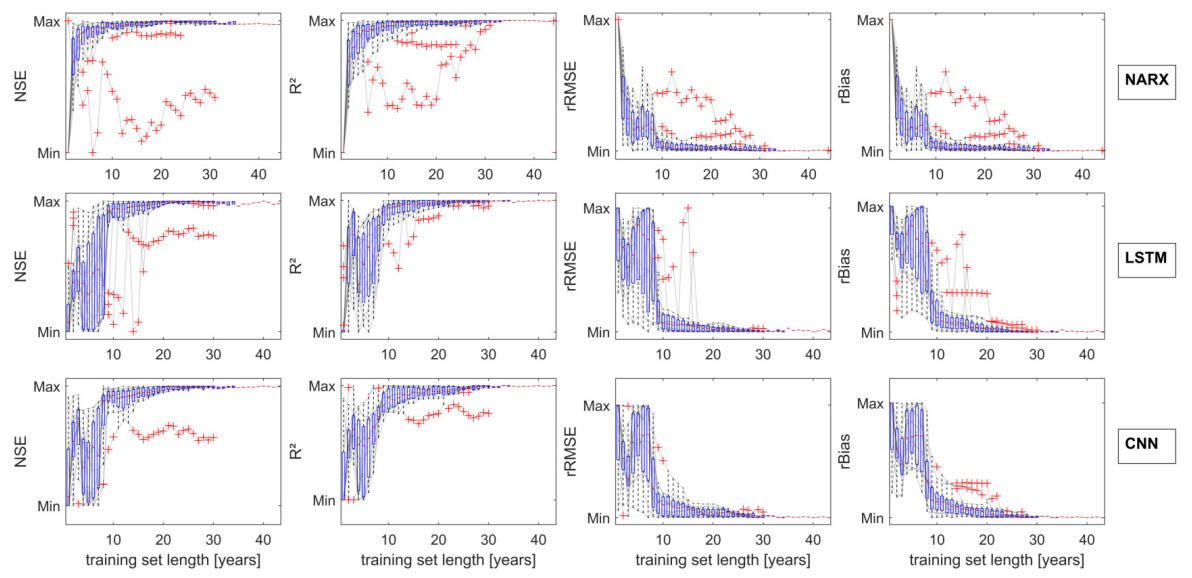

Figure S71: Influence of the available amount of training data on seq2val model performance based on meteorological inputs and past groundwater levels. 

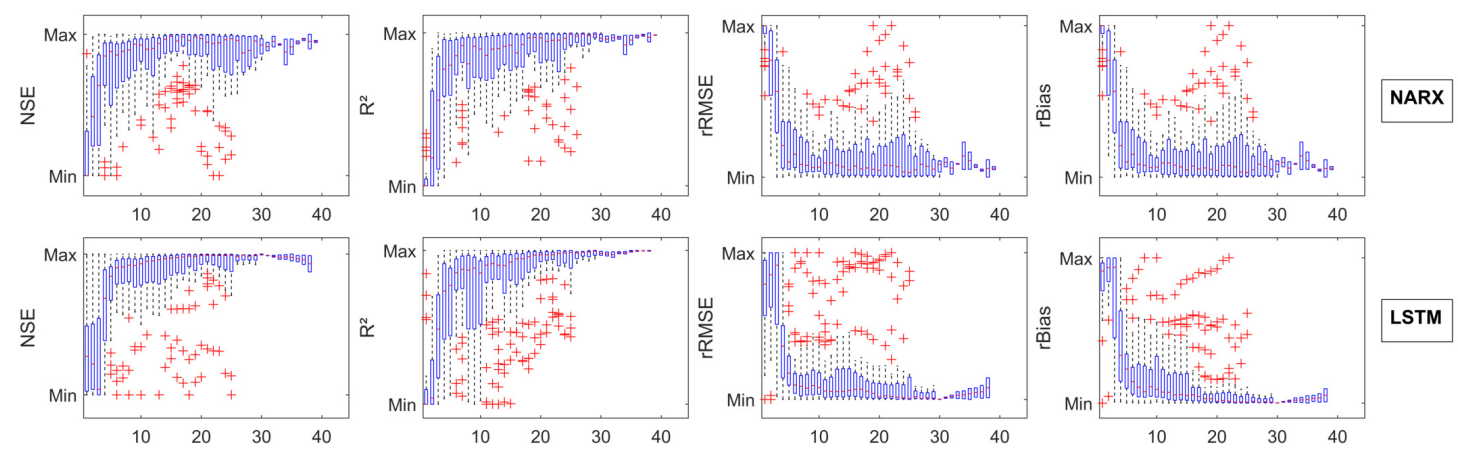

LSTM
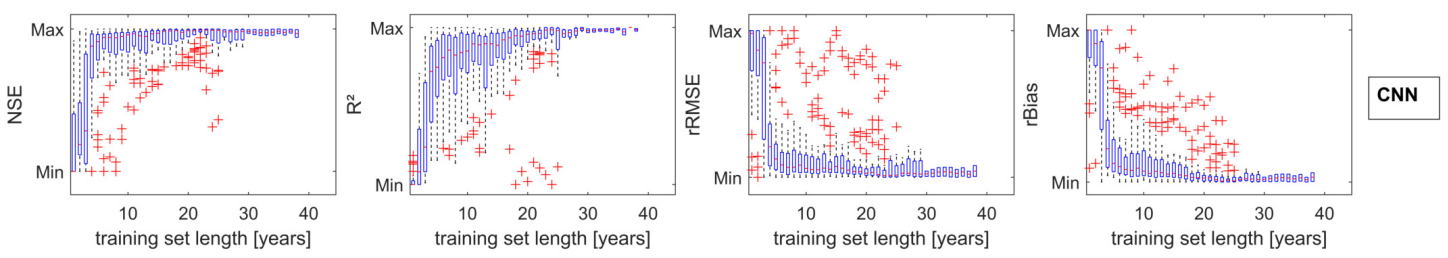

Figure S72: Influence of the available amount of training data on all seq2val model performance with training ending in 2007 instead of 2012
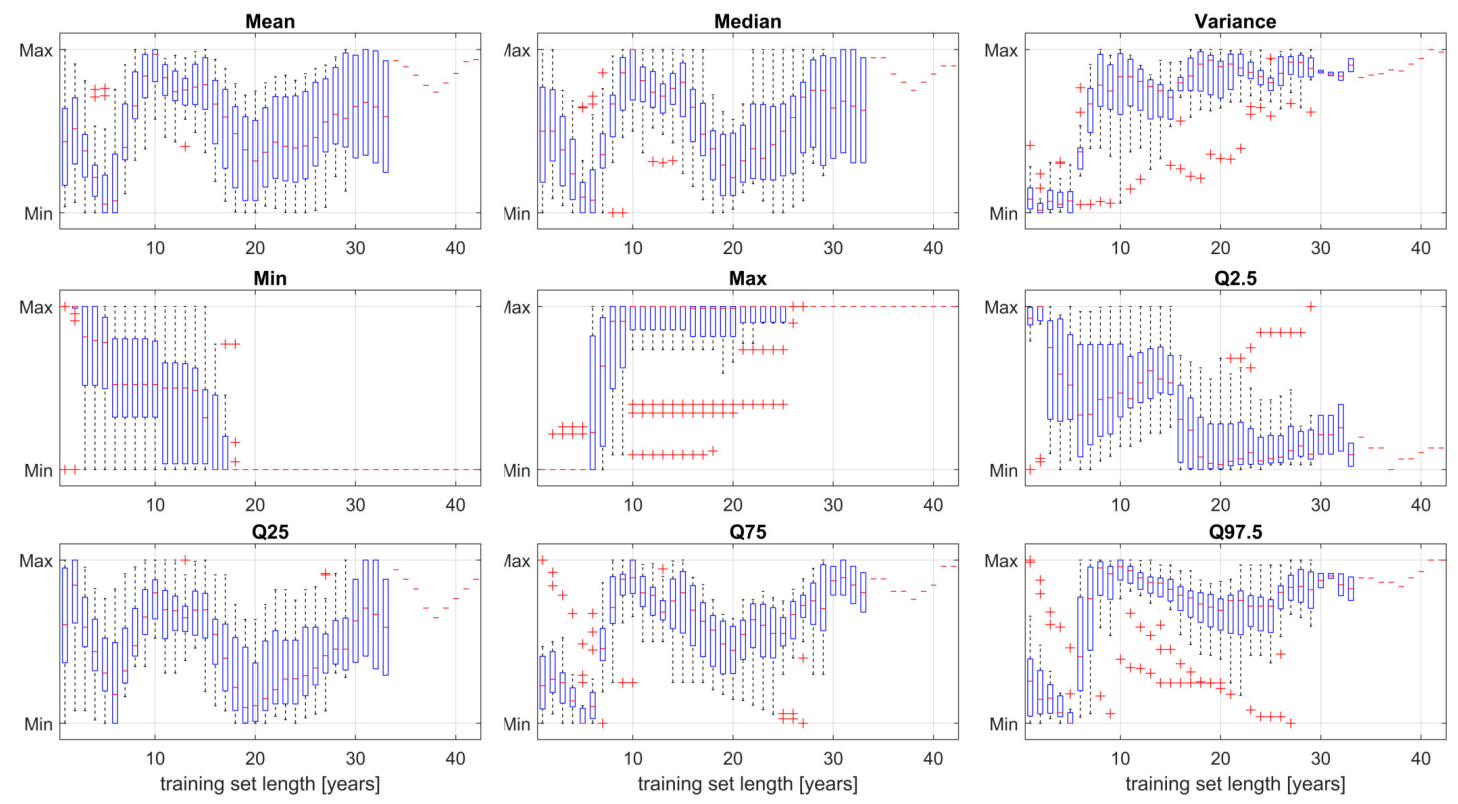

Figure S73: Development of several statistic measures with yearly expanding time series length from 2012 on backwards (Q: Quantile). 